Historic, Archive Document

Do not assume content reflects current scientific knowledge, policies, or practices. 



\subsection{1}

\section{1-1925}

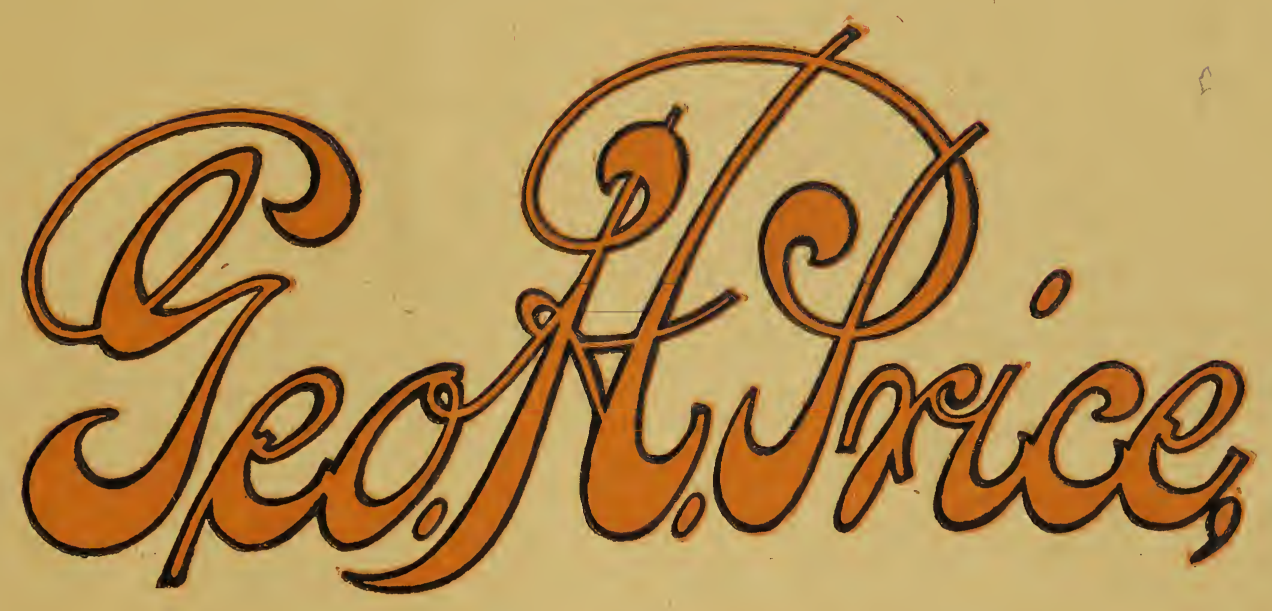

SEEDSMAN ALBANY

NEW YORK

Shoemaker 



\section{THE ALBANY SEED STORE}

Established 1831

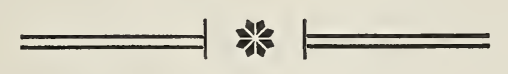

Annual Catalogue

FINE FLOWER,

VEGETABLE

AND FIELD
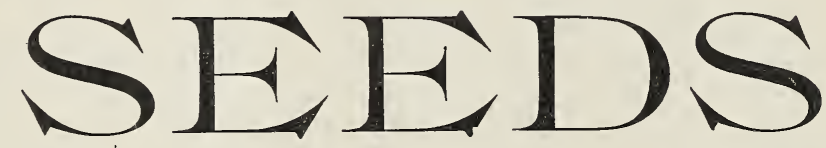

FROM

\section{GEORGE H.PRICE. SEEDSMAN}

Importer, Grower and Dealer in Seed.

Also Dealer in Horticultural and Agricultural Implements FERTILIZERS AND POULTRY SUPPLIES

\section{ALBANY, N. Y.}




\section{NINETY-FOURTH YEAR OF THE ALBANY SEED STORE 1925}

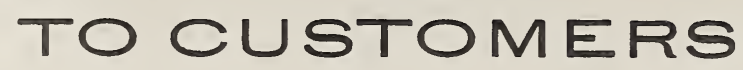

(1) HE SEEDS I offer for sale are of my own growing and importing, and I take the greatest care in their production to have them pure and true to name, testing everything for germination before selling, making a thorough business of it, and never send out anything that does not grow; this, taken in connection with the system of furnishing the best stock seeds and carefully watching the crops, gives my customers the most ample protection. The seeds offered in this Catalogue are of the very best sorts and strains to be obtained; they comprise all the best standard kinds and many new varieties of merit.

Thanking you for your patronage during the past, I hope for a continuance of the same this season.

How to Order. - In writing your order, do it is plainly as possible, and be sure to sign your name distinctly, giving the post-office address, county and state in full, and also express office, for many times it is better to send by express than by mail. Each year we have orders we cannot fill because they are unsigned. Make your order out as soon as possible. It will be an advantage to you and a favor to us, as later in the season prices are liable to advance.

We Fill Orders Promptly.- We aim to fill orders and dispatch them immediately after receipt, unless some special cause prevents, in which case acknowledgment is made and explanation given. Where no shipping instructions are given, we send by the best route possible. Packages too large to send by mail we shall send either by express or freight. We fill an order for ten cents worth of seed with the same promptness and care as one for fifty dollars.

How to Remit.- Send money by post-office or express money order, registered letter or draft. We guarantee the safe arrival of money sent in this way. Postage stamps received as cash when notes are not obtainable, or to make proper change. (Two-cent stamps preferred.)

We Pay the Postage to all parts of the United States on the seeds mentioned in this Catalogue in packets, ounces and one pounds, without adding anything to the list price.

Please Observe that though we prepay the postage on seeds, as above stated, we make no addition to our Catalogue prices to cover this outlay. When ordering in quantities over one pound add postage at parcel post rates.

Remember the mail and express bring our store to your door. It costs no more to buy of us from a distance than if you came into our store and purchased seed over our counter.

Testing.- All our seeds are thoroughly tested in a most careful manner before we sell them, and only those that grow well do we send out. This, in connection with our system of having to do only with the best stocks, and carefully watching the crops, affords ourselves and customers the best protection possible.

My long experience for the past fifty years in the growing, selecting and improving of stocks enables me to supply customers with seeds that cannot be surpassed.

I do not warrant seed, because, as is well known, so many causes may, and often do, prevent the best seeds from growing and producing crops, from no fault of the seeds. We, therefore, in sommon with all other reliable seedsmen, wish it distinctly understood that, while we do not send out any seeds that we do not believe to be of the best quality, we sell no seeds with warranty, express or implied, in any respect, and will not be in any way responsible for the crop. If the purchaser does not accept the seeds on these terms they must be returned at once, and the money that has been paid for the same will be refunded. 


\section{WE WISH TO CALL YOUR ATTENTION TO THE FOLLOWING VARIE-} TIES OF SPECIAL MERIT FOR THE HOME GARDEN.

PRICE'S IMPROVED GOLDEN WAX BEANS (ROUND POD). A splendid, tender, stringless variety.

PRICE'S ALBANI BEET. Best early turnip shaped for table use.

PRICE'S EARLY HORN CARROT. The roots are orange red, about three inches long; very early.

PRICE'S DANVERS CARROT. Smooth, solid, splendid flavor; best variety for general crop.

PRICE'S FANCY GOLDEN SELF BLEACHING CELERY. Best variety for late Summer or early Fall.

PRICE'S ALBANY MARKET CELERY. An excellent Winter variety.

PRICE'S SUMMER DANISH CABBAGE. Best variety for late Summer and early Fall.

PRICE'S SPECIAL STRAIN TRUCKERS FAVORITE CABBAGE. Best Winter variety.

PRICE'S EARLY DAYBREAK CORN. The earliest and largest of all white cob corns.

PRICE'S EARLY GIANT CORN. Very large and second early.

PRICE'S COLOSSAL SWEET CORN. Early, large, very sweet and excellent garden variety.

PRICE'S BUTTERCUP SWEET CORN. As early as Golden Bantam; larger ears; very sweet; a beautiful golden color.

PRICE'S GOLDEN EVERGREEN CORN. A general crop golden variety, large ears, deep kernels; a splendid table variety.
PRICE'S HYBRID CUCUMBER. Very uniform, good green color, and very prolific. A splendid pickling variety.

PRICE'S EXCELSIOR CUCUMBER. Early, dark green in color; very productive; splendid pickle variety.

PRICE'S SILESIA LETTUCE. Very curled and tender; fine for early and general crop; one of the best for market and family use.

PRICE'S WHITE BIG BOSTON LETTUCE. An early solid heading variety, crisp and tender.

THE BAKER MUSK MELON. Salmon fleshed; hard shell; a delicious table melon.

THE BENDER IMELON. Salmon color; deep fleshed; hard shell; an excellent shipper.

PRICE'S IIMPROVED YELLOW GLOBE DANVERS ONION. An excellent keeper; heavy yielder; uniform in shape and color.

PRICE'S IMPROVED TELEPHONE PEAS. A very fine variety for general crop; large dark green pods; very prolific.

PRICE'S GIANT SWEET PEPPER. Very sweet; large and uniform fruits; ripens quite early; bright scarlet color and very prolific.

PRICE'S IDEAL HOLLOW CROWN PARSNIP. Smooth, sweet and large; a very fine garden variety.

PRICE'S EARLY SUNRISE POTATOES. A splendid yielder and an excellent cooker.

PRICE'S EARLY SUPERB TOMATO. As early as Bonny Best; larger, more solid; very uniform and ripens evenly.

PRICE'S NEW PINK TOMATO. Early, smooth and very prolific.

\section{SPECIALTIES IN FLOWER SEEDS}

PRICE'S GIANT FLOWERED MIXTURE OF BRANCHING ASTERS. Flowers are most beautifully formed, large and double, in a variety of bright colors.

PRICE'S SPECIAL MIXTURE OF DWARF NASTURTIUMS. A fine mixture of large flowering varieties in many colors.

PRICE'S SUPERB MIXED PANSY. Large flowers, fine colors with brilliant markings.
'PRICE'S SUPERB MIXTURE OF SWEET PEAS. An unsurpassable mixture of bright colors in exquisite shades; large flowering type.

PRICE'S GIANT FLOWERED ZINNIAS. Large double flowers of perfect shape; brilliant colors of compact growth.

PRICES SPECIAL MIXTURE OF WILD GARDEN FLOWER SEED. An interesting variety of flowers in brilliant colors. 


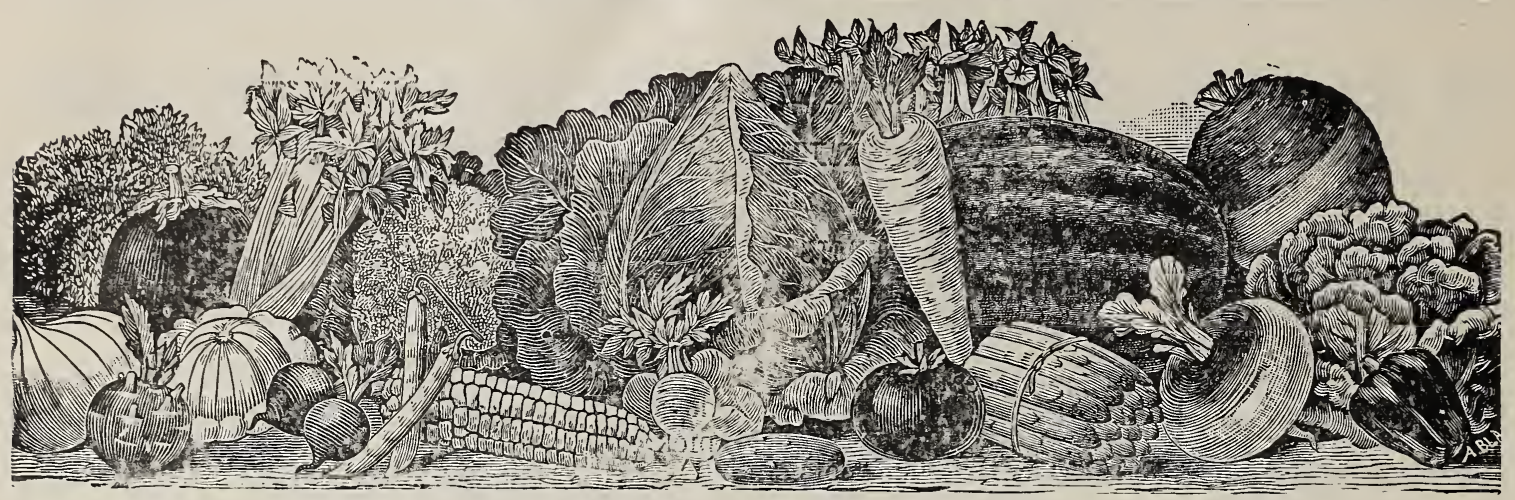

\title{
VEGETABLE SEEDS
}

\section{ASPARAGUS}

Sow the seed in the Spring as early as the ground will permit, in rows one foot apart. Cultivate and keep free from weeds. When two years old transplant to the permanent beds. Roots should be set fourteen to sixteen inches apart in the row and a distance of two and one-half or three feet between the rows. Set fourteen or sixteen inches deep and cover gradually.

\section{ASPARAGUS ROOTS (Two Years Old)}

Washington, Giant of Argenteuil, Palmetto, Barr's Mammoth and Reading Giant. By. the 100 or 1000. Price on application.

Packets of the following varieties sufficient for ten hills or eight feet of drill, 10c each.

\section{BEANS - DWARF GREEN PODDED (Best Garden Varieties)}

\begin{abstract}
EXTRA EARLY RED VALENTINE.............. $\frac{1}{4}$ lb. $15 \mathrm{c} ; \frac{1}{2}$ lb. 20c; 1 lb. 30c; 2 lbs. 55c BLACK VALENTINE ................... $\frac{1}{4} \mathrm{lb} .15 \mathrm{c} ; \frac{1}{2} \mathrm{lb} .20 \mathrm{c} ; 1 \mathrm{lb} .35 \mathrm{c} ; 2 \mathrm{lbs} .60 \mathrm{c}$ EARLY SIX WEEKS. ................... $\frac{1}{4} \mathrm{lb} .15 \mathrm{c} ; \frac{1}{2} \mathrm{lb} .20 \mathrm{c} ; 1 \mathrm{lb} .30 \mathrm{c} ; 2$ lbs. $55 \mathrm{c}$ BOUNTIFUL. . . ........................ $\frac{1}{4}$ lb. $15 \mathrm{c} ; \frac{1}{2} \mathrm{lb} .20 \mathrm{c} ; 1 \mathrm{lb} .35 \mathrm{c} ; 2 \mathrm{lbs} .60 \mathrm{c}$ GIANT STRINGLESS GREEN POD.............. $\frac{1}{4} \mathrm{lb} .15 \mathrm{c} ; \frac{1}{2} \mathrm{lb} .20 \mathrm{c} ; 1 \mathrm{lb} .35 \mathrm{c} ; 2 \mathrm{lbs} .60 \mathrm{c}$ BURPEE'S STRINGLESS GREEN POD ............. $\frac{1}{4}$ lb. 15c; 表 lb. $20 \mathrm{c} ; 1 \mathrm{lb} .35 \mathrm{c} ; 2 \mathrm{lbs} .60 \mathrm{c}$ FULL MEASURE ...................... $\frac{1}{4} \mathrm{lb} .15 \mathrm{c} ; \frac{1}{2} \mathrm{lb} .25 \mathrm{c} ; 1 \mathrm{lb} .35 \mathrm{c} ; 2 \mathrm{lbs} .60 \mathrm{c}$ EXTRA EARLY REFUGEE................. $\frac{1}{4} \mathrm{lb} .15 \mathrm{c} ; \frac{1}{2} \mathrm{lb} .20 \mathrm{c} ; 1 \mathrm{lb} .30 \mathrm{c} ; 2 \mathrm{lbs} .55 \mathrm{c}$ REFUGEE OR 1000 T0 1.................. $\frac{1}{4}$ lb. 15c; $\frac{1}{2}$ lb. 20c; 1 lb. 30c; 2 lbs. 55c HODSON GREEN POD....................... $\frac{1}{4}$ lb. 15c; $\frac{1}{2}$ lb. $20 \mathrm{c} ; 1 \mathrm{lb} .30 \mathrm{c} ; 2$ lbs. $55 \mathrm{c}$ RUBY DWARF HORTICULTURAL (cranberry) ........ $\frac{1}{4} \mathrm{lb} .20 \mathrm{c} ; \frac{1}{2} \mathrm{lb} .25 \mathrm{c} ; 1 \mathrm{lb} .35 \mathrm{r} ; 2 \mathrm{lbs} .60 \mathrm{c}$ FRENCH HORTICULTURAL (cranberry) ........... $\frac{1}{4} \mathrm{lb} .20 \mathrm{c} ; \frac{1}{2} \mathrm{lb} .25 \mathrm{c} ; 1 \mathrm{lb}$ 35c: $2 \mathrm{lbs} .60 \mathrm{c}$ WHITE MARROWFAT .................. Price on application.
\end{abstract}

WHITE KIDNEY .................... Price on application.

BEANS - DWARF WAX OR YELIOW PODDED (Best Garden Varieties) DAVIS KIDNEY WAX...................... $\frac{1}{4}$ lb. 15c; $\frac{1}{2}$ lb. 20c; 1 lb. 30c; 2 lbs. 55c WARDWELL'S KIDNEY WAX ................... $\frac{1}{4}$ lb. 15c; $\frac{1}{2}$ lb. 20c; 1 lb. 30c; 2 lbs. 55c ROUND POD KIDNEY WAX................... $\frac{1}{4}$ lb. $15 \mathrm{c} ; \frac{1}{2}$ lb. $25 \mathrm{c} ; 1 \mathrm{lb} .35 \mathrm{c} ; 2$ lbs. 60c CURRIE'S RUST PROOF WAX................. $\frac{1}{4}$ lb. 15c; $\frac{1}{2} \mathrm{lb} .20 \mathrm{c} ; 1 \mathrm{lb} .30 \mathrm{c} ; 2 \mathrm{lbs} .55 \mathrm{c}$ IMPROVED GOLDEN WAX................... $\frac{1}{4} \mathrm{lb} .15 \mathrm{c} ; \frac{1}{2} \mathrm{lb} .20 \mathrm{c} ; 1 \mathrm{lb} .30 \mathrm{c} ; 2 \mathrm{lbs} .55 \mathrm{c}$ PRICE'S IMPROVED GOLDEN WAX (round pod)...... $\frac{1}{4}$ lb. 20c; $\frac{1}{2}$ lb. 25c; 1 lb. 35c; 2 lbs. 60c UNRIVALED WAX ...................... $\frac{1}{4} \mathrm{lb} .15 \mathrm{c} ; \frac{1}{2} \mathrm{lb} .25 \mathrm{c} ; 1 \mathrm{lb} .35 \mathrm{c} ; 2 \mathrm{lbs} .60 \mathrm{c}$ SURE CROP WAX...................... PENCIL POD BLACK WAX ................... $\frac{1}{4}$ lb. $15 \mathrm{c} ; \frac{1}{2} \mathrm{lb} .20 \mathrm{c} ; 1 \mathrm{lb} .30 \mathrm{c} ; 2$ lbs. $55 \mathrm{c}$ GERMAN WAX OR BUTTER BUSH.............. $\frac{1}{4}$ lb. 15c; $\frac{1}{2} \mathrm{lb} .20 \mathrm{c} ; 1 \mathrm{lb} .30 \mathrm{c} ; 2 \mathrm{lbs} .55 \mathrm{c}$

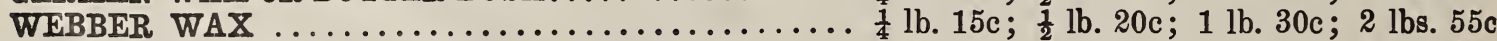
BRITTLE WAX ........................ INEW KIDNEY WAX.............. ...... $\frac{1}{4}$ lb. 15c; $\frac{1}{2}$ lb. 25c; 1 lb. $35 \mathrm{c} ; 2 \mathrm{lbs} .60 \mathrm{c}$ HODSON WAX ........................ $\frac{1}{4}$ lb. 15c; $\frac{1}{2} \mathrm{lb} .20 \mathrm{c} ; 1 \mathrm{lb} .30 \mathrm{c} ; 2$ lbs. 55c 
BEANS - DWARF OR BUSH LIMA (Best Garden Varieties)

FORDHOOK BUSH LIMA.

BURPEE'S IMPROVED BUSH LIMA

NEW WONDER BUSH LIMA.

WOOD'S PROLIFIC BUSH LIMA.

HENDERSON'S BUSH LIMA. .

\section{BEANS - POLE OR RUNNING VARIETIES}

HORTICULTURAL OR SPECKLED CRANBERRY...... per pkt. $10 \mathrm{c} ; \frac{1}{4} \mathrm{lb} .15 \mathrm{c} ; \frac{1}{2} \mathrm{lb} .20 \mathrm{c} ; 1 \mathrm{~b} .35 \mathrm{c}$

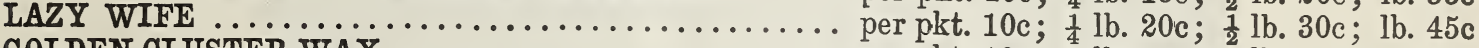
GOLDEN CLUSTER WAX................... per pkt. 10c; $\frac{1}{4} \mathrm{lb} .20 \mathrm{c} ; \frac{1}{2} \mathrm{lb} .30 \mathrm{c} ; 1 \mathrm{~b} .45 \mathrm{c}$ KENTUCKY WONDER (wax pod) ............... per pkt. 10c; $\frac{1}{4}$ lb. $15 \mathrm{c} ; \frac{1}{2} \mathrm{lb} .25 \mathrm{c} ; 1 \mathrm{lb} .40 \mathrm{c}$ KENTUCKY WONDER (green pod)............... per pkt. 10c; $\frac{1}{4} 1 \mathrm{lb} .15 \mathrm{c} ; \frac{1}{2} \mathrm{lb} .25 \mathrm{c}$; lb. 40c

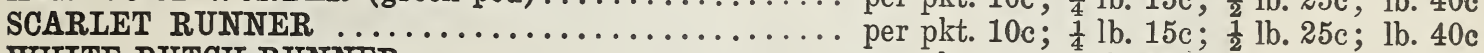
WHITE DUTCH RUNNER.................. per pkt. 10c; $\frac{1}{4} \mathrm{lb} .15 \mathrm{c} ; \frac{1}{2} \mathrm{lb} .25 \mathrm{c} ; \mathrm{lb} .40 \mathrm{c}$ SEIBERT'S EARLY LIMA. .................. per pkt. $10 \mathrm{c} ; \frac{1}{4} \mathrm{lb} .15 \mathrm{c} ; \frac{1}{2} \mathrm{lb} .25 \mathrm{c} ;$ lb. 40c EARLY LEVIATHAN LIIIA. ................. per pkt. $10 \mathrm{c} ; \frac{1}{4} \mathrm{lb} .15 \mathrm{c} ; \frac{1}{2} \mathrm{lb} .25 \mathrm{c} ; \mathrm{lb} .40 \mathrm{c}$ KING OF GARDEN LIMA ................. per pkt. $10 \mathrm{c} ; \frac{1}{4} \mathrm{lb} .15 \mathrm{c} ; \frac{1}{2} \mathrm{lb} .20 \mathrm{c} ; \mathrm{lb} .30 \mathrm{c}$ FORD'S MAMMOTH POD LIMA............... per pkt. $10 \mathrm{c} ; \frac{1}{4} \mathrm{lb} .15 \mathrm{c} ; \frac{1}{2}$ lb. $25 \mathrm{c} ; \mathrm{lb} .40 \mathrm{c}$

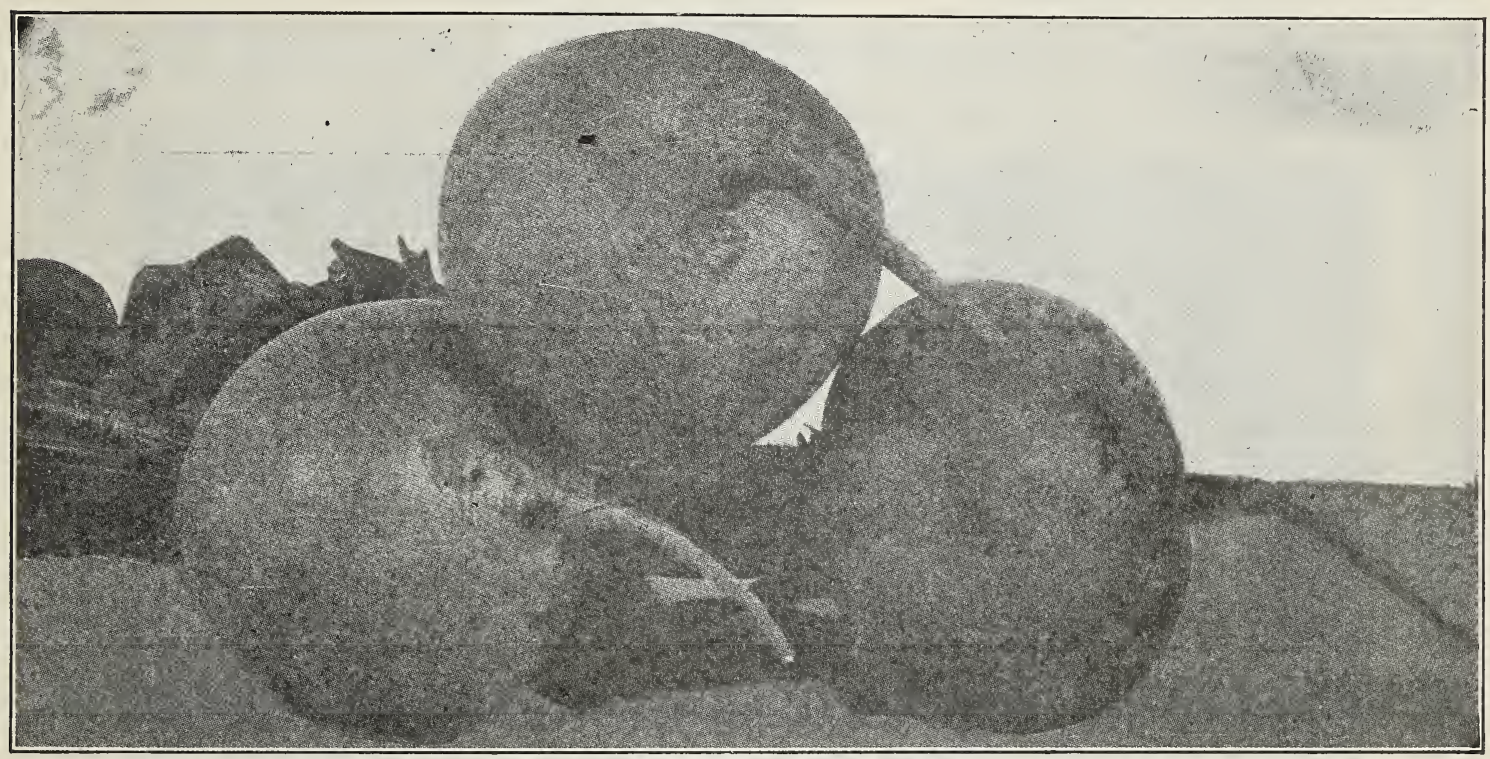

DETROIT DARK RED BEET

BEETS - BEST TABLE VARIETIES

Packets of the following varieties, $5 \mathrm{c}$ each.

PRICE'S ALBANI . ..................... ounce 15c; $\frac{1}{4} \mathrm{lb} .40 \mathrm{c} ; \frac{1}{2} \mathrm{lb} .70 \mathrm{c} ; \mathrm{lb} . \$ 1.25$

EARLY ECLIPSE ..................... ounce $15 \mathrm{c} ; \frac{1}{4} \mathrm{lb} .35 \mathrm{c} ; \frac{1}{2} \mathrm{lb} .60 \mathrm{c} ; 1 \mathrm{lb} .1 .00$

EXTRA EARLY LENTZ . . . . . . . . . . . . . . . . ounce $15 \mathrm{c} ; \frac{1}{4} \mathrm{lb} .35 \mathrm{c} ; \frac{1}{2} \mathrm{lb} .60 \mathrm{c} ; \mathrm{lb} .1 .00$

EXTRA EARLY EGYPTIAN. ................ ounce $15 \mathrm{c} ; \frac{1}{4} \mathrm{lb} .35 \mathrm{c} ; \frac{1}{2} \mathrm{lb} .60 \mathrm{c} ; \mathrm{lb} .1 .00$

EXTRA EARLY CROSBY EGYPTIAN............ ounce $15 \mathrm{c} ; \frac{1}{4} \mathrm{lb} .35 \mathrm{c} ; \frac{1}{2} \mathrm{lb} .60 \mathrm{c} ; \mathrm{lb} .1 .00$

EARIY BLOOD TURNIP. .................. ounce $15 \mathrm{c} ; \frac{1}{4} \mathrm{lb} .35 \mathrm{c} ; \frac{1}{2} \mathrm{lb} .60 \mathrm{c} ; 1 \mathrm{lb} .1 .00$

CRIMSON GLOBE ...................... ounce $15 \mathrm{c} ; \frac{1}{4} \mathrm{lb} .35 \mathrm{c} ; \frac{1}{2} \mathrm{lb} .60 \mathrm{c} ; \mathrm{lb} .1 .00$

ARLINGTON BLOOD TURNIP................ ounce $15 \mathrm{c} ; \frac{1}{4} \mathrm{lb} .30 \mathrm{c} ; \frac{1}{2} \mathrm{lb} .50 \mathrm{c} ; \mathrm{lb} .90$

DETROIT DARK RED.................... ounce $15 \mathrm{c} ; \frac{1}{4} \mathrm{lb} .35 \mathrm{c} ; \frac{1}{2} \mathrm{lb} .60 \mathrm{c} ; \mathrm{lb} .1 .00$

EDMUNDS' BL00D TURNIP................. ounce $15 \mathrm{c} ; \frac{1}{4} \mathrm{lb} .35 \mathrm{c} ; \frac{1}{2} \mathrm{lb} .60 \mathrm{c} ; \mathrm{lb} .1 .00$

NUTTING GEM ...................... ounce $15 \mathrm{c} ; \frac{1}{4} \mathrm{lb} .35 \mathrm{c} ; \frac{1}{2} \mathrm{lb} .60 \mathrm{c} ; \mathrm{lb} .1 .00$

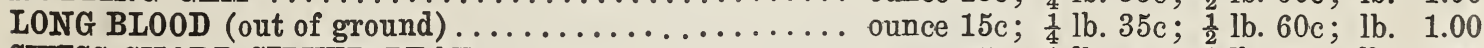

SWISS CHARD SILVER LEAF............... ounce $15 \mathrm{c} ; \frac{1}{4} \mathrm{lb} .40 \mathrm{c} ; \frac{1}{2} \mathrm{lb} .70 \mathrm{c} ; \mathrm{lb} .1 .25$

SWISS CHARD GIANT LUCULLUS. ............. ounce $15 \mathrm{c} ; \frac{1}{4} \mathrm{lb} .40 \mathrm{c} ; \frac{1}{2} \mathrm{lb} .70 \mathrm{c} ; \mathrm{lb} .1 .25$ 


\section{STOCK AND SUGAR BEETS}

Six pounds will sow one acre. Special price in large quantities.

VILMORIN'S IMPROVED WHITE SUGAR......... ounce $10 \mathrm{c} ; \frac{1}{4} \mathrm{lb} .25 \mathrm{c} ; \frac{1}{2} \mathrm{lb} .40 \mathrm{c} ; 1 \mathrm{lb} .70 \mathrm{c}$ LANE'S IMPERIAL WHITE SUGAR............... ounce $10 \mathrm{c} ; \frac{1}{4} \mathrm{lb} .25 \mathrm{c} ; \frac{1}{2} \mathrm{lb} .45 \mathrm{c} ; 1 \mathrm{lb} .80 \mathrm{c}$ MAMMOTH LONG RED MANGLE WURZEL........... ounce $10 \mathrm{c} ; \frac{1}{4} \mathrm{lb} .20 \mathrm{c} ; \frac{1}{2} \mathrm{lb} .35 \mathrm{c} ; 1 \mathrm{lb} .60 \mathrm{c}$

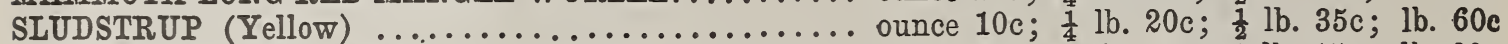
GOLDEN TANKARD MANGLE WURZEL............ ounce $10 \mathrm{c} ; \frac{1}{4} \mathrm{lb} .25 \mathrm{c} ; \frac{1}{2} \mathrm{lb} .45 \mathrm{c} ; 1 \mathrm{~b} .80 \mathrm{c}$ GIANT HALF SUGAR GREEN TOP............. ounce $10 \mathrm{c} ; \frac{1}{4} \mathrm{lb} .25 \mathrm{c} ; \frac{1}{2} \mathrm{lb} .45 \mathrm{c} ; 1 \mathrm{lb} .80 \mathrm{c}$

\section{BRUSSEL SPROUTS}

Sow same as Cabbage through May and transplant in July.

INIPROVED DWARF LONG ISLAND......... per pkt. $10 \mathrm{c} ; \frac{1}{2}$ Oz. 20c; Oz. 30c; $\frac{1}{4}$ lb. $\$ 1.25$; $\frac{1}{2}$ lb. $\$ 2.25 ;$ lb. $\$ 4.00$.

\section{CARROT}

PRICE'S EARLY HORN. ............. per pkt. $5 \mathrm{c} ;$ oz. $20 \mathrm{c} ; \frac{1}{4} \mathrm{lb} .40 \mathrm{c} ; \frac{1}{2} \mathrm{lb} .70 \mathrm{c} ; 1 \mathrm{lb} . \$ 1.25$ OXHEART OR GUERANDE.............. per pkt. $5 \mathrm{c} ;$ oz. $15 \mathrm{c} ; \frac{1}{4} \mathrm{lb} .30 \mathrm{c} ; \frac{1}{2} \mathrm{lb} .55 \mathrm{c} ; 1 \mathrm{lb} .1 .00$ HALF LONG SCARLET NANTES......... per pkt. $5 \mathrm{c} ;$ oz. $20 \mathrm{c} ; \frac{1}{4} \mathrm{lb} .40 \mathrm{c} ; \frac{1}{2} \mathrm{lb} .70 \mathrm{c} ; 1 \mathrm{~b} .1 .25$

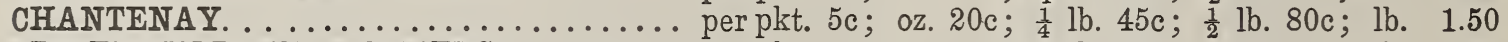
PRICE'S IMPROVED DANVERS.......... per pkt. $5 \mathrm{c} ;$ oz. $20 \mathrm{c} ; \frac{1}{4} \mathrm{lb} .45 \mathrm{c} ; \frac{1}{2} \mathrm{lb} .80 \mathrm{c} ; 1 \mathrm{~b} .1 .50$ INTERMEDIATE OR ST. VALERY........ per pkt. $5 \mathrm{c} ;$ oz. $15 \mathrm{c} ; \frac{1}{4} \mathrm{lb} .35 \mathrm{c} ; \frac{1}{2} \mathrm{lb} .60 \mathrm{c} ; 1 \mathrm{~b} .1 .00$ PRICE'S BOSTONIAN .............. per pkt. $5 \mathrm{c}$; oz. $25 \mathrm{c} ; \frac{1}{4} \mathrm{lb} .45 \mathrm{c} ; \frac{1}{2} \mathrm{lb} .80 \mathrm{c} ; 1 \mathrm{~b} .1 .50$ WHITE BELGIAN ................. per pkt. $5 \mathrm{c} ;$ oz. $15 \mathrm{c} ; \frac{1}{4} \mathrm{lb} .35 \mathrm{c} ; \frac{1}{2} \mathrm{lb} .60 \mathrm{c} ; 1 \mathrm{lb} .1 .00$

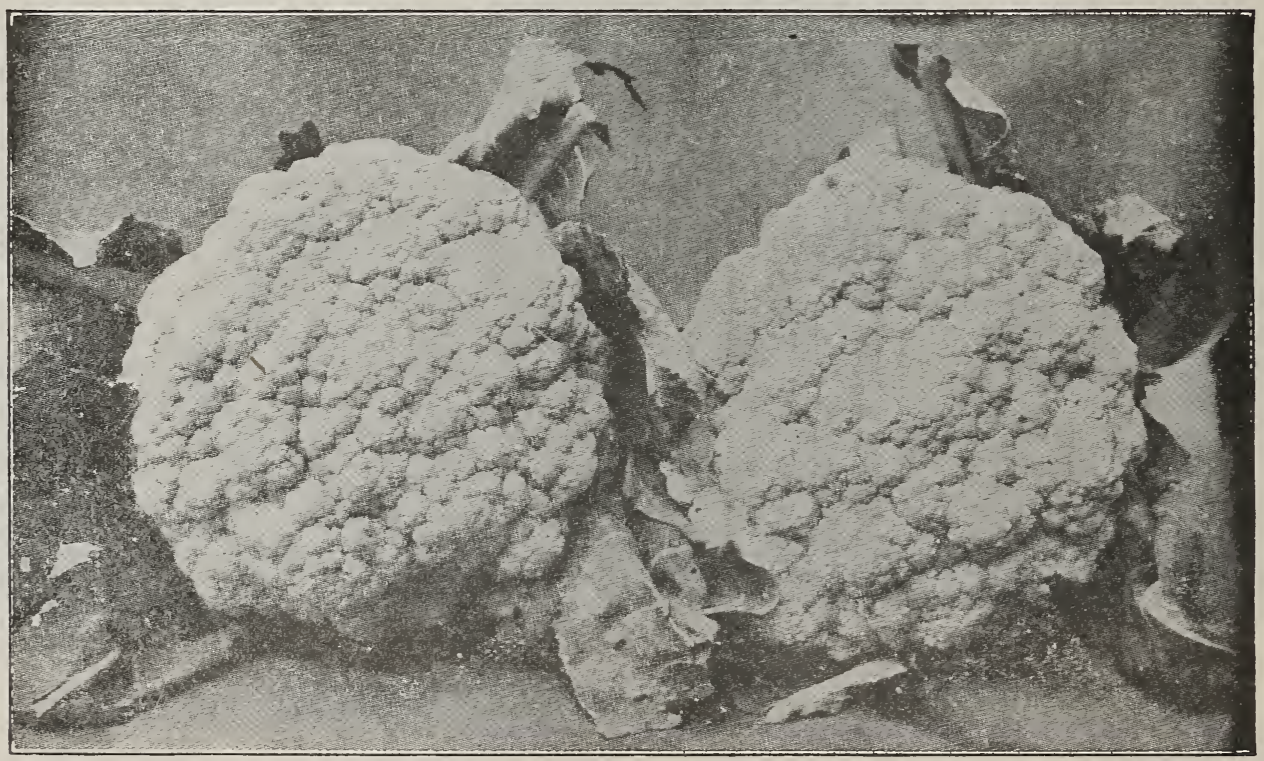

\section{CAULIFLOWER}

Seed can be sown under glass for early and outdoors for general crop, and transplanted the same as Cabbage.

PRICE'S PEDIGREE SNOWBALL. The earliest of all Snowball, the most dwarf, of compact growth and a very sure header. Will stand dry weather better than any other variety of Snowball Cauliflower.

PRICE'S PEDIGREE SNOWBALL........... per pkt. $25 \mathrm{c} ; \frac{1}{2}$ Oz. $\$ 2.00 ;$ oz. $\$ 3.50 ; \frac{1}{4}$ lb. $\$ 12.00$ SELECTED SNOWBALL . . . . . . . . . . . . . . . . . per pkt. 20 c ; $\frac{1}{2}$ oz. $1.50 ;$ oz. $2.50 ; \frac{1}{4}$ lb. 8.00 EXTRA SELECTED DWARF ERFURT........ per pkt. $25 \mathrm{c} ; \frac{1}{2}$ oz. $2.00 ;$ oz. $3.00 ; \frac{1}{4}$ lb. 8.00 PRICE'S DANISH GIANT. . . . . . . . . . . . per pkt. $25 \mathrm{c} ; \frac{1}{2}$ oz. $2.00 ;$ oz. $3.50 ; \frac{1}{4}$ lb. 10.00 LARGE LATE DRY WEATHER........... per pkt. $25 \mathrm{c} ; \frac{1}{2}$ oz. $1.75 ;$ oz. $3.00 ; \frac{1}{4}$ lb. 8.00

\section{CRESS AND CORN SALAD}

CRESS OR PEPPERGRASS...... pkt. 5c; $\frac{1}{2}$ oz. $10 \mathrm{c} ;$ oz. $15 \mathrm{c} ; \frac{1}{4} \mathrm{lb} . \$ 0.40 ; \frac{1}{2} \mathrm{lb} . \$ 0.70 ; 1 \mathrm{lb} . \$ 1.25$ CRESS, TRUE WATER.......... pkt. $10 \mathrm{c} ; \frac{1}{2}$ Oz. $30 \mathrm{c} ;$ Oz. $50 \mathrm{c} ; \frac{1}{4} \mathrm{lb} .1 .40 ; \frac{1}{2} \mathrm{lb} .2 .50 ; 1 \mathrm{lb} .5 .00$ CORN SALAD OR FETTICUS. ... pkt. $5 \mathrm{c} ; \frac{1}{2}$ oz. $10 \mathrm{c} ;$ oz. $15 \mathrm{c} ; \frac{1}{4} \mathrm{lb} . \quad .45 ; \frac{1}{2} \mathrm{lb} . \quad .80 ; 1 \mathrm{lb} .1 .50$ 


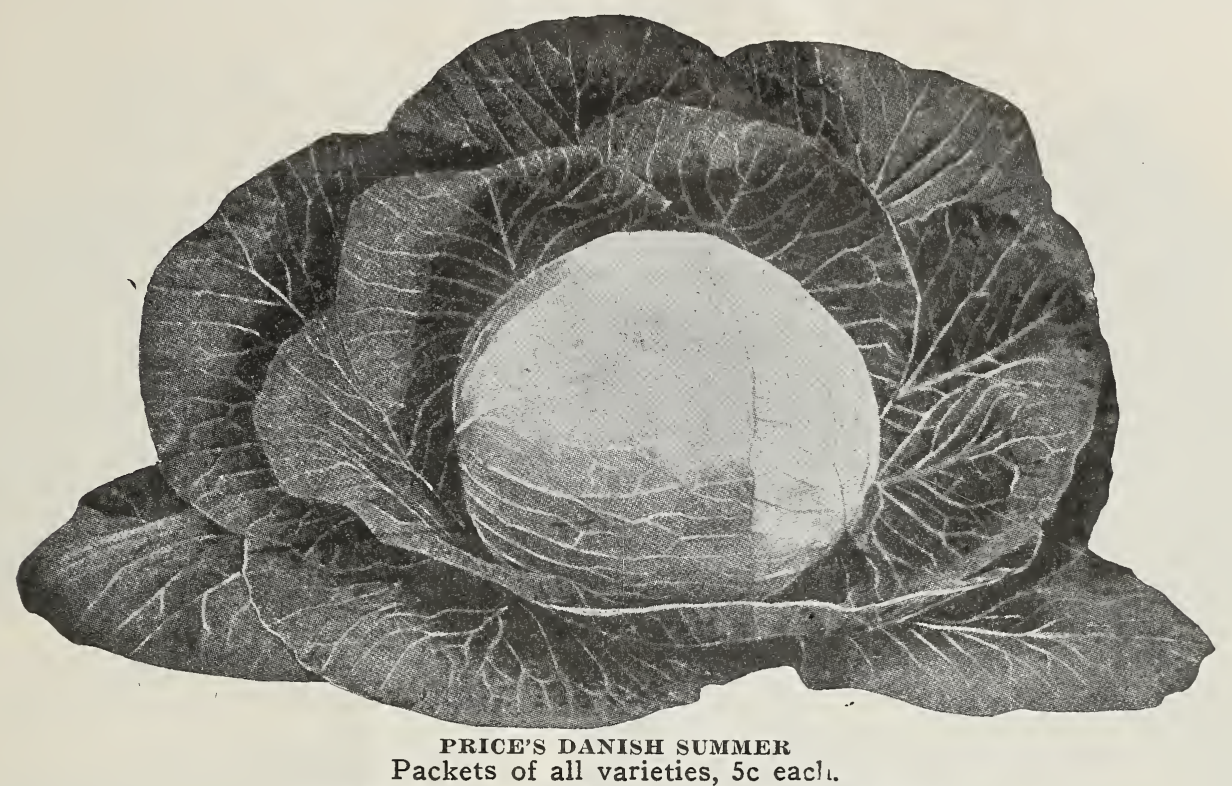

CABBAGE - EARLY VARIETIES

EARLY JERSEY WAKEFIELD $\quad \ldots . . . . . . . . .1 \frac{1}{2}$ oz. $25 \mathrm{c} ;$ oz. $40 \mathrm{c} ; \frac{1}{4} \mathrm{lb} . \$ 1.00 ; 1 \mathrm{lb} . \$ 3.50$ CHARLESTON WAKEFIELD ................. $\frac{1}{2}$ oz. $25 \mathrm{c} ;$ oz. $40 \mathrm{c} ; \frac{1}{4} \mathrm{lb} .1 .00 ; 1 \mathrm{lb} .3 .50$ EARLY WINNINGSTADT $\ldots \ldots \ldots \ldots \ldots \ldots \ldots \ldots$. $\frac{1}{2}$ oz. $20 \mathrm{c} ;$ Oz. $30 \mathrm{c} ; \frac{1}{4} \mathrm{lb} . .85 ; 1 \mathrm{lb} .3 .00$ COPENHAGEN MARKET .................. $\frac{1}{2}$ oz. $25 \mathrm{c} ;$ oz. $40 \mathrm{c} ; \frac{1}{4} \mathrm{lb} .1 .25 ; 1 \mathrm{lb} .4 .50$ PEERLESS......................... $\frac{1}{2}$ oz. $25 \mathrm{c} ;$ oz. $40 \mathrm{c} ; \frac{1}{4} \mathrm{lb} .1 .25 ; 1 \mathrm{lb} .4 .00$ EXTRA EARLY EXPRESS................. $\frac{1}{2}$ oz. $25 \mathrm{c} ;$ oz. $40 \mathrm{c} ; \frac{1}{4} \mathrm{lb} .1 .25 ; 1 \mathrm{lb} .4 .00$

\section{SECOND EARLY OR LATE SUMMER VARIETIES}

HENDERSON'S SUMIMER ................. $\frac{1}{2}$ oz. 20c; oz. $30 \mathrm{c} ; \frac{1}{4} 1 \mathrm{lb} . \$ 0.85 ; 1 \mathrm{lb} . \$ 3.00$ ALL HEAD (early) ................... $\frac{1}{2}$ oz. $25 \mathrm{c} ;$ oz. $40 \mathrm{c} ; \frac{1}{4} \mathrm{lb} .1 .00 ; 1 \mathrm{lb} .3 .50$ SUCCESSION. . . .................... $\frac{1}{2}$ oz. $20 \mathrm{c} ;$ oz. $30 \mathrm{c} ; \frac{1}{4} \mathrm{lb} . \quad .85 ; 1 \mathrm{lb} .3 .00$ ALL SEASONS $\ldots \ldots \ldots \ldots \ldots \ldots \ldots \ldots \ldots \ldots \ldots, \frac{1}{2}$ oz. $25 \mathrm{c} ;$ oz. $40 \mathrm{c} ; \frac{1}{4} \mathrm{lb} .1 .00 ; 1 \mathrm{lb} .3 .50$ SURE HEAD PRICE'S PREMIUM DRUMHEAD PRICE'S FLAT DUTCH.

PRICE'S SUMMIMER DANISH. GLORY OF ENKHUIZEN. oz. $20 \mathrm{c} ;$ oz. $30 \mathrm{c} ; \frac{1}{4} \mathrm{lb}$. $\frac{1}{2}$ oz. $20 \mathrm{c}$; oz. $30 \mathrm{c} ; \frac{1}{4} \mathrm{lb}$. $\frac{1}{2}$ oz. $20 \mathrm{c} ;$ oz. $30 \mathrm{c} ; \frac{1}{4} \mathrm{lb}$. $\frac{1}{2}$ oz. $25 \mathrm{c} ;$ oz. $40 \mathrm{c} ; \frac{1}{4} \mathrm{lb} .1 .25 ; 1 \mathrm{lb} .4 .00$ $\frac{1}{2}$ oz. $25 \mathrm{c}$; oz. $4.0 \mathrm{c}$; $\frac{1}{4} \mathrm{lb} .1 .00 ; 1 \mathrm{lb} .3 .50$

FALL OR WINTER VARIETIES

DANISH ROUND HEAD (short stem)........... $\frac{1}{2}$ oz. $25 \mathrm{c} ;$ oz. $40 \mathrm{c} ; \frac{1}{4}$ lb. $\$ 1.25 ; 1 \mathrm{lb} . \$ 4.00$ DANISH BALL HEAD (tall stem) ............... $\frac{1}{2}$ oz. $25 \mathrm{c} ;$ oz. $40 \mathrm{c} ; \frac{1}{4} \mathrm{lb}: 1.25 ; 1 \mathrm{lb} .4 .00$ TRUCKERS' FAVORITE (middle stem)........... $\frac{1}{2}$ oz. $25 \mathrm{c} ;$ oz. $45 \mathrm{c} ; \frac{1}{4} \mathrm{lb} .1 .35 ; 1 \mathrm{lb} .5 .00$ HOLLANDER (middle stem) ................. $\frac{1}{2}$ oz. $25 \mathrm{c} ;$ oz. $40 \mathrm{c} ; \frac{1}{4} \mathrm{lb} .1 .25 ; 1 \mathrm{lb} .4 .00$ PERFECTION DRUMHEAD SAVOY.............. $\frac{1}{2}$ oz. $25 \mathrm{c} ;$ oz. $40 \mathrm{c} ; \frac{1}{4} \mathrm{lb} .1 .00 ; 1 \mathrm{lb} .3 .50$ CHINESE IMPROVED OR PETSAI............... $\frac{1}{2}$ oz. $15 \mathrm{c} ;$ oz. $25 \mathrm{c} ; \frac{1}{4} \mathrm{lb} . \quad 80 ; 1 \mathrm{lb} .3 .00$ ERFURT LARGE RED DRUMHEAD............ $\frac{1}{2}$ oz. $25 \mathrm{c} ;$ oz. $40 \mathrm{c} ; \frac{1}{4} \mathrm{lb} .1 .25 ; 1 \mathrm{lb} .4 .00$ RED DANISH BALL HEAD ................. $\frac{1}{2}$ oz. $25 \mathrm{c} ; \mathrm{oz} .40 \mathrm{c} ; \frac{1}{4} \mathrm{lb} .1 .00 ; 1 \mathrm{lb} .3 .50$ MAMIMOTH RED ROCK................... $\frac{1}{2}$ oz. $25 \mathrm{c} ;$ oz. $40 \mathrm{c} ; \frac{1}{4} \mathrm{lb} .1 .00 ; 1 \mathrm{lb} .3 .50$

CHICORY, WITLOOF OR FRENCH ENDIVE. The seed should be sown during June in the open ground in drills twelve to fourteen inches apart, in deep rich soil. Allow the plants to grow until November, keeping free from weeds and well cultivated. Then take up the roots and trim off the leaves to about one inch from the neck; break off all side shoots so to leave the root smooth, resembling a smooth parsnip root. Cut off roots at bottom, leaving them a uniform length, eight to ten inches. Plant roots in upright position in a trench sixteen to eighteen inches deep, two inches apart. This will leave the top of the roots about eight inches below the level of the ground. Fill the trench with very light loose soil. (Black fine sand, if possible.) Then cover the trench with eight or ten inches of light manure. This will cause a quick growth and well blanched heads.. per pkt. 10c; $\frac{1}{2}$ oz. $15 \mathrm{c} ;$ oz. $25 \mathrm{c} ; \frac{1}{4} \mathrm{lb} .80 \mathrm{c} ; \frac{1}{2} \mathrm{lb} . \$ 1.50 ; 1 \mathrm{lb} . \$ 2.00$ 


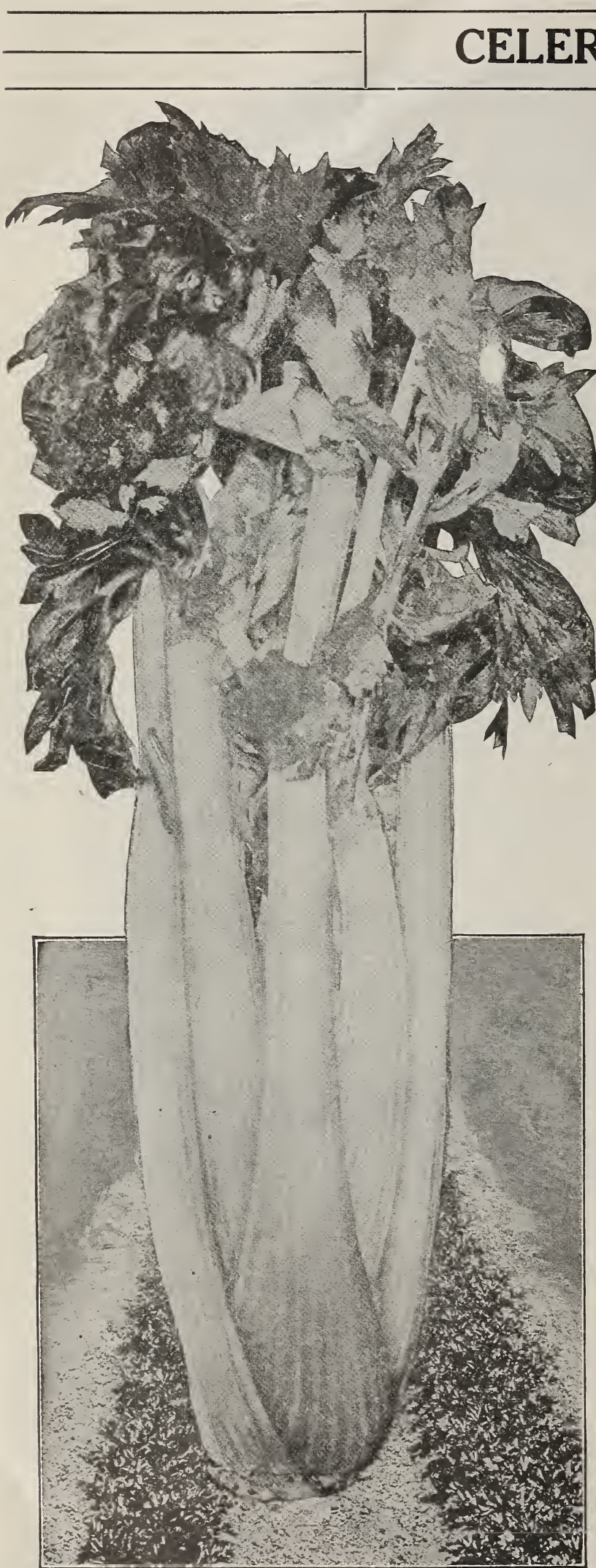

\section{CULTURE}

Should be sown in the Spring in the open ground or in the hot bed. When four or five inches high set in straight rows about five inches apart. The rows should be three or three and one-half feet apart. Water and protect until well rooted. When large enough to blanch, close the stalks and draw the soil up, using care not to get the soil in the center of the heart or stalks.

BEST EARLY OR SUMMER VARIETIES

PRICE'S GOLDEN SELF BLANCHING

(French), pkt. 10c; $\frac{1}{2}$ oz. $45 \mathrm{c}$; oz. 80c; $\frac{1}{4}$ lb. $\$ 2.75 ; \frac{1}{2}$ lb. $\$ 5.25$; lb. $\$ 10.00$.

GOLDEN SELF BLANCHING (American), pkt. $15 \mathrm{c}$; $\frac{1}{2}$ oz. $40 \mathrm{c}$; oz. $70 \mathrm{c}$; $\frac{1}{4}$ lb. $\$ 2.25$; $\frac{1}{2}$ lb. $\$ 4.25$; lb. $\$ 8.00$.

EAS $^{\top}$ BLANCHING (fancy), pkt. $10 \mathrm{c} ; \frac{1}{2} \mathrm{oz}$. 90c; 0z. $\$ 1.25 ; \frac{1}{4}$ lb. $\$ 4.50 ; \frac{1}{2}$ lb. $\$ 8.00$; lb. $\$ 16.00$.

PRICE'S BONNIE GREEN (new easy Blanch Type, very solid), pkt. $15 \mathrm{c}$; $\frac{1}{2}$ oz. $80 \mathrm{c}$; oz. $\$ 1.40$; $\frac{1}{4}$ lb. $\$ 5.00 ; \frac{1}{2}$ lb. $\$ 9.50$; lb. $\$ 18.00$. GOLDEN PLUME. Is becoming very popular among the large market gardeners. It is Easy Blanching crossed with Golden Self Blanching a new Golden type of Easy Blanching, less subject to rust and blight, and a stronger and better keeper. Pkt. 25 ; oz. $\$ 4.00$; 2 oz. $\$ \% .50$; $\frac{1}{4}$ lb. $\$ 13.00$.

\section{BEST LATE FALL AND WINTER VARIETIES}

PRICE'S ALBANY MARKET, pkt. 10c; $\frac{1}{2}$ oz. 30 c; oz. 50 c; $\frac{1}{4}$ lb. $\$ 1.40 ; \frac{1}{2}$ lb. $\$ 2.60$; lb. $\$ 5.00$.

PRICE'S WINTER KING. A new variety, plants medium height, thick heavy ribs, can be marketed in November or held until quite late in the winter. Pkt. 10c; $\frac{1}{2}$ Oz. 30c; oz. 50 c; $\frac{1}{4}$ lb. $\$ 1.60 ;$ lb. $\$ 6.00$.

WINTER RELIANCE, pkt. $10 \mathrm{c}$; $\frac{1}{2}$ oz. $25 \mathrm{c}$; oz. 40 c; $\frac{1}{4}$ lb. $\$ 1.25 ; \frac{1}{2}$ lb. $\$ 2.25$; lb. $\$ 4.00$. COLUMBIA, pkt. $10 \mathrm{c} ; \frac{1}{2}$ oz. $35 \mathrm{c} ;$ oz. $60 \mathrm{c}$; $\frac{1}{4}$ lb. $\$ 1.75 ; \frac{1}{2}$ lb. $\$ 3.25$; lb. $\$ 6.00$.

\section{CELERIAC (KNOB CELERY)}

GIANT SIOOTH ROOTED, pkt. 10c; $\frac{1}{2}$ oz. $25 \mathrm{c} ; \quad$ oz. $40 \mathrm{c} ; \frac{1}{4}$ lb. $\$ 1.25$; $\frac{1}{2}$ lb. $\$ 2.25$; lb. $\$ 4.00$.

\section{CELERY PLANTS}

\section{(See Vegetable Plants, page 22)}

Parties growing celery plants for their own use, unless in very large quantities, will generally ind it more satisfactory to buy the plants. We can supply small or large quantities of plants from June 15th to August 1st, and will be pleased to quote price on application. Plants can be carefully packed in wet moss and shipped long distances and will arrive in good condition. 

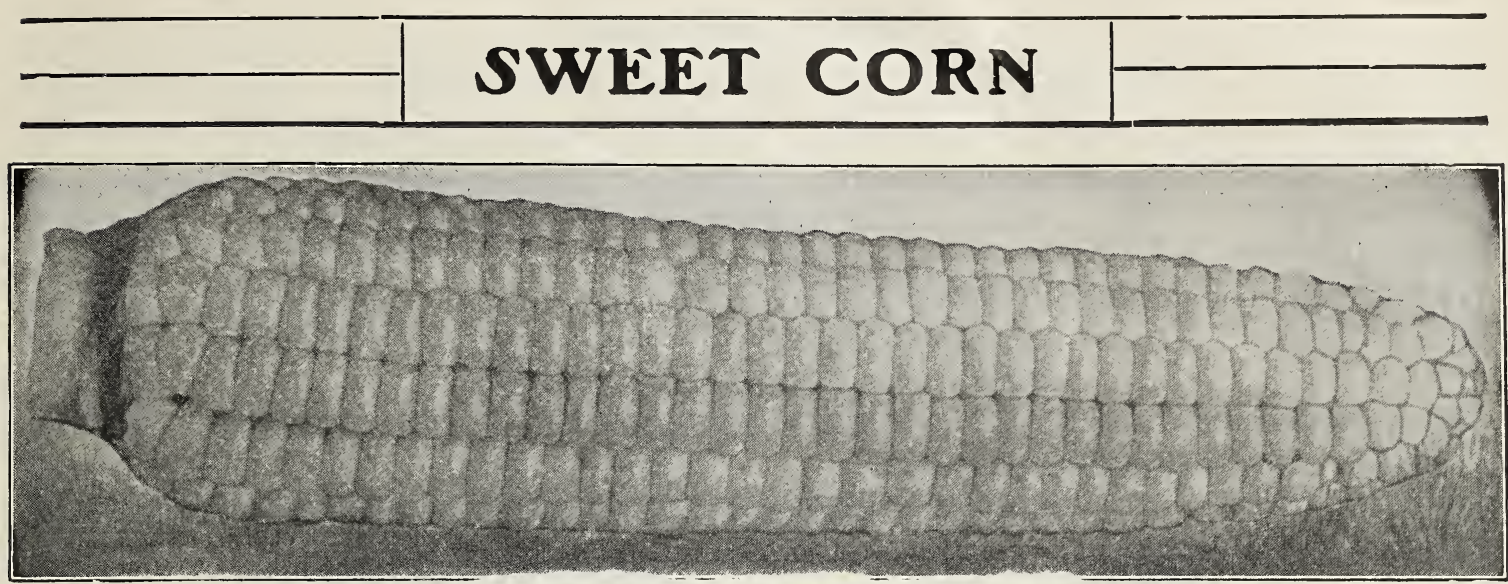

PRICE'S EARLY DAYBREAK SWEET CORN

\section{BEST EXTRA EARLY VARIETIES}

PRICE'S EARLY DAYBREAK.

\section{EARIY DAWN}

MAYFLOWER.

PRICE'S EARLY GIANTT.

EXTRA EARLY WHITE CORY $\frac{1}{1}$ lb. $15 \mathrm{c} ; \frac{1}{2}$ lb. $25 \mathrm{c} ;$ lb. $40 \mathrm{c}$; 2 lbs. $70 \mathrm{c}$ lb. $15 \mathrm{c} ; \frac{1}{2}$ lb. $25 \mathrm{c} ;$ lb. $40 \mathrm{c}$; 2 lbs. $70 \mathrm{c}$ lb. $15 \mathrm{c}$; $\frac{1}{2}$ lb. $20 \mathrm{c}$; lb. $35 \mathrm{c}$; 2 lbs. $60 \mathrm{c}$ lb. $15 \mathrm{c} ; \frac{1}{2}$ lb. $20 \mathrm{c}$; lb. $35 \mathrm{c}$; 2 lbs. $60 \mathrm{c}$ $\frac{1}{4}$ lb. $15 \mathrm{c} ; \frac{1}{2}$ lb. $20 \mathrm{c}$; lb. $30 \mathrm{c} ; 2$ lbs. $50 \mathrm{c}$

\section{BEST SECOND EARLY VARIETIES}

PRICE'S 1900

PRICE'S COLOSSAL

MAIMIOTH WHITE CORY

KENDALL'S EARLY GIANT.

EARLY PROLIFIC

EARLY CROSBY

EARLY MAMMOTH

ASPINWALL.

SHAKER EARLY

PRICE'S PERFECTION

HOWLING MOB $\frac{1}{1}$ lb. $15 \mathrm{c} ; \frac{1}{2}$ lb. $20 \mathrm{c}$

$\frac{1}{1}$ lb. $15 \mathrm{c} ; \frac{1}{2}$ lb. $25 \mathrm{c}$;

$\frac{1}{4}$ lb. $15 \mathrm{c}$;

1 lb. $15 \mathrm{c}$;

$\frac{1}{4}$ lb. $15 \mathrm{c}$;

$\frac{1}{4}$ lb. $15 \mathrm{c}$;

$\frac{1}{1}$ lb. $15 \mathrm{c}$;

$\frac{1}{4}$ lb. $15 \mathrm{c}$;

1 lb. $15 \mathrm{c}$;

1 lb. $15 \mathrm{c}$;

$\frac{1}{4}$ lb. $15 \mathrm{c}$; $\frac{1}{2}$ lb. $20 \mathrm{c}$;

$\frac{1}{2} \mathrm{lb} .20 \mathrm{c}$;

$\frac{1}{2}$ lb. $20 \mathrm{c}$;

$\frac{1}{2}$ lb. $20 \mathrm{c}$;

$\frac{1}{2}$ lb. $20 \mathrm{c}$

$\frac{1}{2}$ lb. $20 \mathrm{c}$;

$\frac{1}{0} \mathrm{lb} .20 \mathrm{c}$

$\frac{1}{2}$ lb. $20 \mathrm{c}$

$\frac{1}{2}$ lb. $20 \mathrm{c}$ lb. $35 \mathrm{c} ; 2$ lbs. $60 \mathrm{c}$

lb. $40 \mathrm{c} ; 2$ lbs. $70 \mathrm{c}$

lb. 30 c; 2 lbs. 50 c

ib. $30 \mathrm{c}$; 2 lbs. $50 \mathrm{c}$

lb. $35 \mathrm{c}$; 2 lbs. $60 \mathrm{r}$

lb. $30 \mathrm{c} ; 2$ lbs. $50 \mathrm{c}$

lb. $30 \mathrm{c} ; 2$ lbs. $50 \mathrm{c}$

lb. $30 \mathrm{c} ; 2$ lbs. $50 \mathrm{c}$

lb. $30 \mathrm{c} ; 2$ lbs. $50 \mathrm{c}$

lb. $35 \mathrm{c} ; 2$ lbs. $60 \mathrm{c}$

lb. $35 \mathrm{c} ; 2$ lbs. $60 \mathrm{c}$

\section{YELLOW OR GOLDEN VARIETIES}

GOLDEN BANTAM

GOLDEN ROD

$\frac{1}{4}$ lb. $15 \mathrm{c}$;

$\frac{1}{1 b} 15 \mathrm{c}$;

$\frac{1}{2}$ lb. $20 \mathrm{c}$;

lb. $35 \mathrm{c} ; 2$ lbs. $60 \mathrm{c}$

PRICE'S BUTTERCUP

$\frac{1}{4}$ lb. $15 \mathrm{c}$;

$\frac{1}{2}$ lb. $25 \mathrm{c}$

$\frac{1}{4}$ lb. $20 \mathrm{c}$

1 lb. $25 \mathrm{c}$

PRICE'S GOLDEN EVERGREEN

BANTAM EVERGREEN

PRICE'S SWEET ORANGE.

CHARLEVOIX.

DE LUE'S GOLDEN GIANT

$\frac{1}{4}$ lb. $15 \mathrm{c}$;

$\frac{1}{4}$ lb. $15 \mathrm{c}$;

$\frac{1}{4}$ lb. $15 \mathrm{c}$;

$\frac{1}{4}$ lb. $20 \mathrm{c}$; lb. $30 \mathrm{c}$; lb. $50 \mathrm{c} ; 2$ lbs. $90 \mathrm{c}$

$\frac{1}{2}$ lb. $20 \mathrm{c} ; 1 \mathrm{~b} .35 \mathrm{c} ; 2$ lbs. $60 \mathrm{c}$

$\frac{1}{2}$ lb. $25 \mathrm{c} ; 1 \mathrm{~b} .40 \mathrm{c} ; 2$ lbs. $70 \mathrm{c}$

$\frac{1}{2}$ lb. $20 \mathrm{c} ; 1 \mathrm{~b} .35 \mathrm{c} ; 2$ lbs. $60 \mathrm{c}$ 1 lb. $30 \mathrm{c} ; \mathrm{lb} .40 \mathrm{c} ; 2$ lbs. $70 \mathrm{c}$

\section{BEST GENERAL CROP VARIETIES}

P. \& R. CHAMPION LONG ISLAND BEAUTY

EARLY EVERGREEN

WHITE EVERGREEN

STOWELL'S EVERGREEN

LATE MAMIMOTH

COUNTRY GENTLEIEN OR SHOE PEG

BLACK MEXICAN

\section{$\frac{1}{4}$ lb. $15 \mathrm{c}$;}

$\frac{1}{4}$ lb. $15 \mathrm{c}$;

$\frac{1}{4}$ lb. $15 \mathrm{c}$;

$\frac{1}{4}$ lb. $15 \mathrm{c}$;

$\frac{1}{4}$ lb. $15 \mathrm{c}$

1 lb. $10 \mathrm{c}$;

$\frac{1}{4}$ lb. $15 \mathrm{c}$

1 lb. $15 \mathrm{c}$ $\frac{1}{2}$ lb. $20 \mathrm{c}$;

$\frac{1}{2}$ lb. $20 \mathrm{c}$;

$\frac{1}{2}$ lb. $20 \mathrm{c}$;

$\frac{1}{2}$ lb. $20 \mathrm{c}$;

$\frac{1}{2} \mathrm{lb} .20 \mathrm{c}$

$\frac{1}{2}$ lb. $15 \mathrm{c}$

$\frac{1}{2} \mathrm{lb} .20 \mathrm{c}$

$\frac{1}{2}$ lb. $20 \mathrm{c}$ lb. $30 \mathrm{c} ; 2$ lbs. $50 \mathrm{c}$ lb. $30 \mathrm{c} ; 2$ lbs. $50 \mathrm{c}$ lb. $35 \mathrm{c} ; 2$ lbs. $60 \mathrm{c}$ lb. $35 \mathrm{c} ; 2$ lbs. $60 \mathrm{c}$ lb. $30 \mathrm{c} ; 2$ lbs. $50 \mathrm{c}$ lb. $25 \mathrm{c} ; 2$ lbs. $45 \mathrm{c}$ lb. $30 \mathrm{c} ; 2$ lbs. $50 \mathrm{c}$ lb. $35 \mathrm{c} ; 2$ lbs. $60 \mathrm{c}$ 


\section{CORN (Continued)}

\section{FLINT CORN, STANDARD VARIETIES}

LONGFELLOW YELLOW FLINT (eight rowed). Large ear, deep kernel, small cob.

EARLY YELLOW CANADA (eight rowed). Medium size ears, very early. COMPTON'S EARLY (twelve rowed). Ears from twelve to fifteen inches in length. SANFORD WHITE FLINT (eight rowed). Large, perfect ears; a heavy yielder. KING PHILIP (eight rowed) (RED). An excellent yielder on poor land.

Prices of the above on application.

\section{DENT OR ENSILAGE CORNS}

EARLY MASTADON, IMIPROVED LEAMING, PRIDE OF THE NORTH, WHITE CAP YELLOW DENT, WHITE EUREKA ENSILAGE.

POP CORN, WHITE RICE, GOLDEN QUEEN, AND RED BEAUTY (0n the ear), $\frac{1}{4} \mathrm{lb} .15 \mathrm{c} ; \frac{1}{2} \mathrm{lb}$. 20 c; lb. 30 c; 2 lbs. $50 \mathrm{c} ; 10 \mathrm{lbs} . \$ 1.70$.

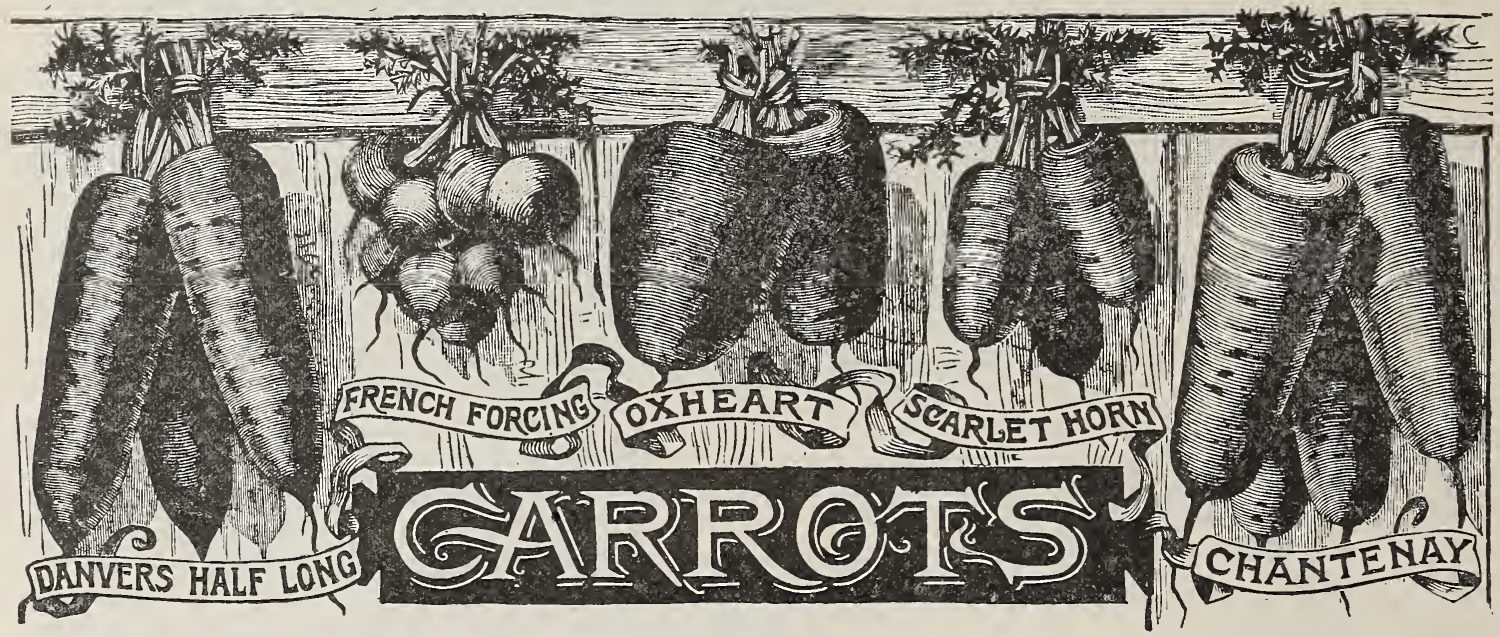

\section{CUCUMBER (Best Garden Varieties)}

Packets of the following varieties, $5 \mathrm{c}$ each.

EARIY RUSSIAN ............................. $15 \mathrm{c} ; \frac{1}{4} \mathrm{lb} .45 \mathrm{c} ; \frac{1}{2} \mathrm{lb} .80 \mathrm{c} ; 1 \mathrm{lb} . \$ 1.50$ EARLY GREEN CLUSTER................... oz. 15c; $\frac{1}{4} \mathrm{lb} .45 \mathrm{c} ; \frac{1}{2} \mathrm{lb} .75 \mathrm{c} ; 1 \mathrm{lb} .1 .40$ IMPROVED WHITE SPINE..................... $15 \mathrm{c} ; \frac{1}{4} \mathrm{lb} .40 \mathrm{c} ; \frac{1}{2} \mathrm{lb} .70 \mathrm{c} ; \mathrm{lb} .1 .30$ ARLINGTON WHITE SPINE................. oz. $15 \mathrm{c} ; \frac{1}{4} \mathrm{lb} .45 \mathrm{c} ; \frac{1}{2} \mathrm{lb} .75 \mathrm{c} ; 1 \mathrm{~b} .1 .40$ EVERGREEN WHITE SPINE...................... $15 \mathrm{c} ; \frac{1}{4} \mathrm{lb} .45 \mathrm{c} ; \frac{1}{2} \mathrm{lb} .80 \mathrm{c} ; 1 \mathrm{~b} .1 .50$ KLONDYKE WHITE SPINE................. Oz. 15c; $\frac{1}{4} \mathrm{lb} .40 \mathrm{c} ; \frac{1}{2} \mathrm{lb} .70 \mathrm{c} ; 1 \mathrm{lb} .1 .30$ PRICE'S HYBRID ........................ oz. $15 \mathrm{c} ; \frac{1}{4} \mathrm{lb} .50 \mathrm{c} ; \frac{1}{2} \mathrm{lb} .85 \mathrm{c} ; \mathrm{lb} .1 .60$

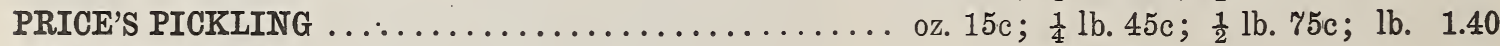
DAVIS PERFECT ............................. 15c; $\frac{1}{4} \mathrm{lb} .50 \mathrm{c} ; \frac{1}{2} \mathrm{lb} .85 \mathrm{c} ; 1 \mathrm{lb} .1 .60$ LONG GREEN ............................. $15 \mathrm{c} ; \frac{1}{4} \mathrm{lb} .55 \mathrm{c} ; \frac{1}{2} \mathrm{lb} .90 \mathrm{c} ; 1 \mathrm{lb} .1 .70$ FORDHOOK FAMOUS ......................... $15 \mathrm{c} ; \frac{1}{4} \mathrm{lb} .45 \mathrm{c} ; \frac{1}{2} \mathrm{lb} .80 \mathrm{c} ; \mathrm{lb} .1 .50$ JAPANESE CLIMBING ................... oz. $20 \mathrm{c} ; \frac{1}{4} \mathrm{lb} .60 \mathrm{c}$ WEST INDIA GHERKIN.................... oz. $20 \mathrm{c} ; \frac{1}{4} \mathrm{lb} .60 \mathrm{c}$

BLACK BEAUTY .................... per pkt. 10c; $\frac{1}{2}$ oz. 40c; oz. 70c; $\frac{1}{4}$ lb. $\$ 2.25$ NEW YORK IIMPROVED PURPLE............. per pkt. 10c; $\frac{1}{2}$ oz. 30c; oz. 50c; $\frac{1}{4}$ lb. 2.00 FLORIDA HIGH BUSH. ................. per pkt. $10 \mathrm{c} ; \frac{1}{2}$ oz. $35 \mathrm{c} ;$ oz. $60 \mathrm{c} ; \frac{1}{4} \mathrm{lb} .2 .00$ 


\section{ENDIVE}

GREEN CURLED

WHITE CURLED

BROAD LEAVED per pkt. $5 \mathrm{c} ; \frac{1}{2}$ oz. $10 \mathrm{c} ;$ oz. $20 \mathrm{c} ; \frac{1}{4}$ lb. $50 \mathrm{c}$ per pkt. $5 \mathrm{c} ; \frac{1}{2}$ oz. $10 \mathrm{c} ;$ oz. $20 \mathrm{c} ; \frac{1}{4}$ lb. $50 \mathrm{c}$ per pkt. $5 \mathrm{c} ; \frac{1}{2}$ oz. $10 \mathrm{c} ;$ oz. $20 \mathrm{c} ; \frac{1}{4}$ lb. $45 \mathrm{c}$

\section{KALE}

DWARF GREEN CURLED ................... per pkt. 5c; $\frac{1}{2}$ oz. 15c; oz. 20c; $\frac{1}{4}$ lb. 50c DWARF MOSS CURLED.................... per pkt. $5 \mathrm{c} ; \frac{1}{2}$ oz. $15 \mathrm{c}$; oz. $20 \mathrm{c}$; $\frac{1}{4} \mathrm{lb} .50 \mathrm{c}$ GREEN CURLED SCOTCH (tall) ............... per pkt. $5 \mathrm{c} ; \frac{1}{2}$ oz. 15c; oz. 20c; $\frac{1}{4}$ lb. 40c SIBERIAN OR WINTER SPROUTS............. per pkt. $5 \mathrm{c} ; \frac{1}{2}$ oz. $15 \mathrm{c} ;$ oz. $20 \mathrm{c} ; \frac{1}{4}$ lb. $40 \mathrm{c}$

\section{KOHL RABI}

EARIY WHITE VIENNA SHORT LEAVED......... per pkt. 10c; $\frac{1}{2}$ oz. $15 \mathrm{c} ;$ oz. $25 \mathrm{c} ; \frac{1}{4}$ lb. $\$ 1.00$ EARLY PURPLE VIENNA................. per pkt. 10c; $\frac{1}{2}$ oz. $15 \mathrm{c} ;$ oz. $25 \mathrm{c} ; \frac{1}{4} 1 \mathrm{~b} .1 .00$

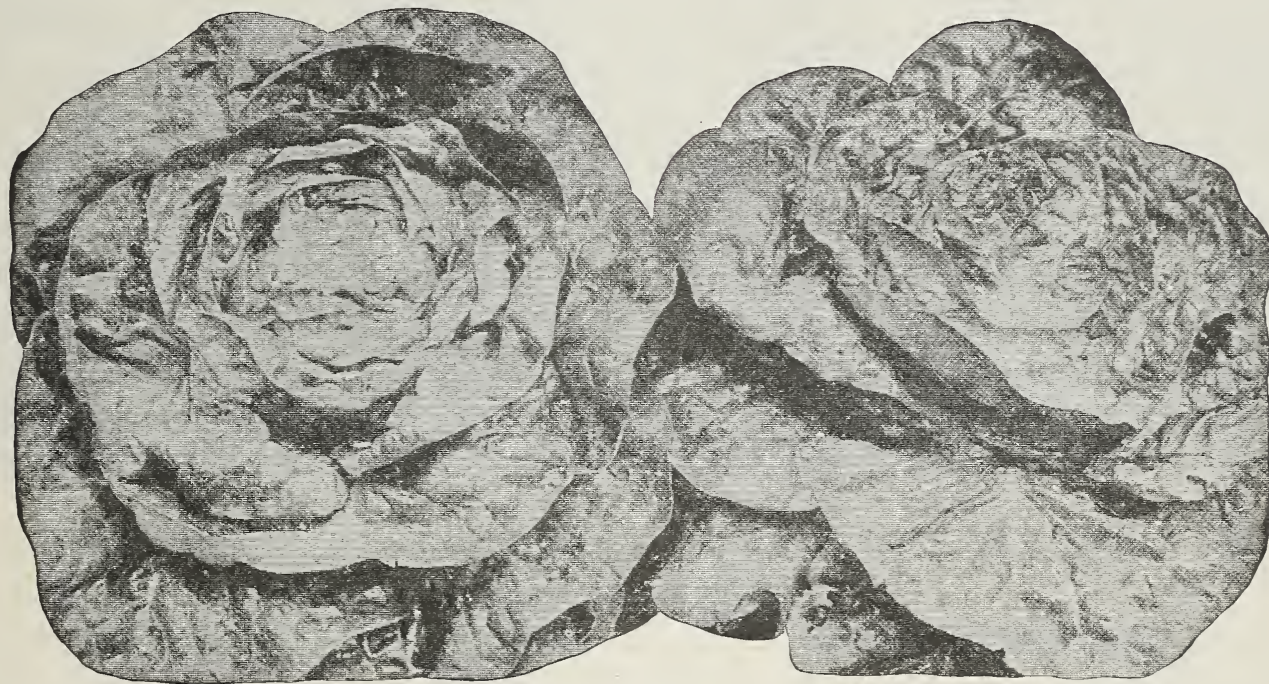

PRICE'S MAMMOTH HEAD LETTUCE

\section{LETTUCE - CURLED OR CUTTING VARIETIES}

PRICE'S IMPROVED CURIED SILESIA............ per pkt. $5 \mathrm{c} ; \frac{1}{2}$ oz. $10 \mathrm{c} ;$ oz. $20 \mathrm{c} ; \frac{1}{1 \mathrm{~b} .} 50 \mathrm{c}$ EARLY CURLED SIMPSON.................... per pkt. 5c; $\frac{1}{2}$ 0z. 10c; 0z. 20c; $\frac{1}{4} 1 \mathrm{lb} .50 \mathrm{c}$ BLACK SEEDED SIMPSON. .................. per pkt. $5 \mathrm{c} ; \frac{1}{2}$ oz. 10c; oz. 20c; $\frac{1}{4} 1 \mathrm{lb} .50 \mathrm{c}$ GRAND RAPIDS per pkt. $5 \mathrm{c} ; \frac{1}{2}$ oz. $10 \mathrm{c} ;$ oz. $20 \mathrm{c} ; \frac{1}{4}$ lb. $50 \mathrm{c}$

\section{HEADING VA.RIETIES}

ICEBERG. ......................... per pkt. $5 \mathrm{c} ; \frac{1}{2}$ oz. $10 \mathrm{c} ;$ oz. $20 \mathrm{c} ; \frac{1}{4} \mathrm{lb} .60 \mathrm{c}$

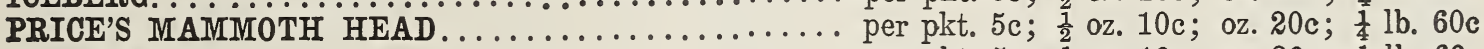

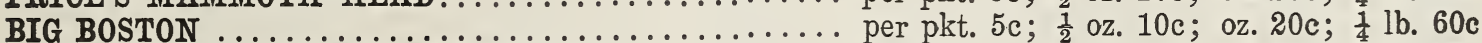
MAMMOTH BLACK SEEDED BUTTER............. per pkt. $5 \mathrm{c} ; \frac{1}{2}$ oz. $10 \mathrm{c} ;$ oz. 20c; $\frac{1}{4} 1 \mathrm{~b} .50 \mathrm{c}$ IMPROVED SALAMANDER .................. per pkt. $5 \mathrm{c} ; \frac{1}{2}$ oz. 10c; oz. 20c; $\frac{1}{4}$ lb. 50c

MAY KING

PRIZEHEAD.

NEW YORK OR WONDERFUL

ONRIVALED SUMMER

WAYAHEAD.

ALL SEASONS

HANSON.

MIGNONETTE

CALIFORNIA CREAM BUTTER

COS OR ROMAINE. per pkt. 5c; per pkt. $5 \mathrm{c}$; per pkt. 5c; per pkt. $5 \mathrm{c}$; per pkt. $5 \mathrm{c}$; per pkt. $5 \mathrm{c}$; per pkt. $5 \mathrm{c}$; per pkt. $5 \mathrm{c}$; per pkt. $5 \mathrm{c}$; per pkt. $5 c$;
Zz. 10c;

Oz. $10 \mathrm{c} ;$

0z. $20 \mathrm{c} ; 4$ 1b. $50 \mathrm{c}$ 0z. 10c; 0z. 20c; 专 lb. 60c Oz. $10 \mathrm{c} ;$ oz. $20 \mathrm{c} ; \frac{1}{4}$ lb. $50 \mathrm{c}$ oz. $10 \mathrm{c} ;$ oz. $20 \mathrm{c} ; \frac{1}{4}$ lb. $60 \mathrm{c}$ oz. $10 \mathrm{c} ;$ oz. $20 \mathrm{c} ; \frac{1}{4} \mathrm{lb} .50 \mathrm{c}$ oz. $10 \mathrm{c} ;$ oz. $20 \mathrm{c} ; \frac{1}{4} \mathrm{lb} .50 \mathrm{c}$ Oz. $10 \mathrm{c} ;$ oz. $20 \mathrm{c} ; \frac{1}{4}$ lb. $60 \mathrm{c}$ 0z. $10 \mathrm{c} ;$ oz. $20 \mathrm{c} ; \frac{1}{4}$ lb. $60 \mathrm{c}$ $\frac{1}{2}$ oz. $10 \mathrm{c} ;$ oz. $20 \mathrm{c} ; \frac{1}{4}$ lb. $50 \mathrm{c}$ 


\section{LEEK}

BEST FLAG .......................... per pkt. 5c; $\frac{1}{2}$ oz. $15 \mathrm{c} ;$ oz. $35 \mathrm{c} ; \frac{1}{4}$ lb. $80 \mathrm{c}$

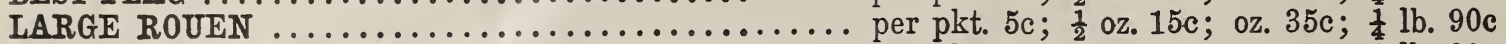
MONSTROUS CARENTAN .................. per pkt. 5c; $\frac{1}{2}$ oz. 15c; oz. 35c; $\frac{1}{4}$ lb. $90 \mathrm{c}$

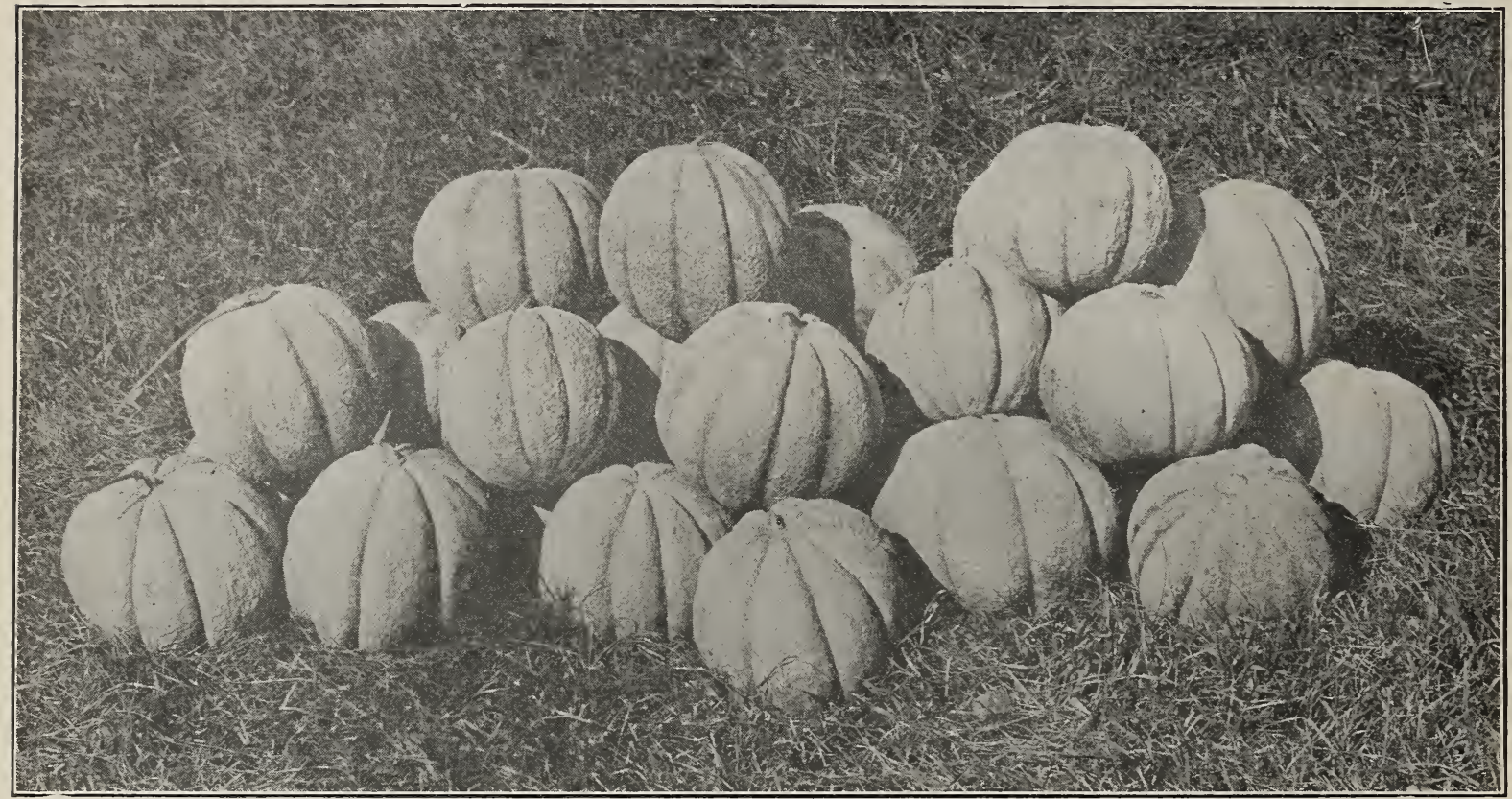

THE CELFBRATED BAKER MELON. Seed Raised at Grand Vlew Farm.

Deep Salmon Flesh, Very Heavy Net, Flavor Unsurpassed, Heavy Shell, an Excellent Shipper

\section{MUSK MELON}

THE CELEBRATED BAKER MELON ... per pkt. $15 \mathrm{c} ;$ oz. $50 \mathrm{c} ; \frac{1}{4} \mathrm{lb} . \$ 1.60 ; \frac{1}{2} \mathrm{lb} . \$ 3.00 ; 1 \mathrm{lb} . \$ 6.00$ BENDER IMELON ............... per pkt. $15 \mathrm{c} ;$ oz. $50 \mathrm{c} ; \frac{1}{4} \mathrm{lb} .1 .60 ; \frac{1}{2} \mathrm{lb} .3 .00 ; \mathrm{lb} .6 .00$

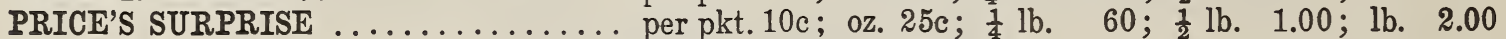

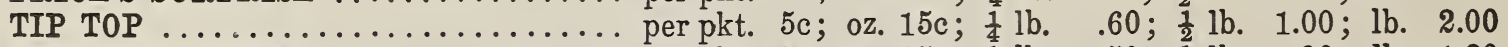
MILLER'S CREAM ............. per pkt. $5 \mathrm{c} ;$ oz. $15 \mathrm{c} ; \frac{1}{4} \mathrm{lb} . \quad .50 ; \frac{1}{2} \mathrm{lb} . \quad .90 ; \mathrm{lb} .1 .80$ EXTRA EARLY HACKENSACK. . . . . . . per pkt. $5 \mathrm{c}$; oz. $15 \mathrm{c} ; \frac{1}{4} \mathrm{lb} . \quad .60 ; \frac{1}{2} \mathrm{lb} .1 .00 ; 1 \mathrm{~b} .2 .00$ EMERALD GEMI .............. per pkt. $5 \mathrm{c} ;$ oz. $15 \mathrm{c} ; \frac{1}{4} \mathrm{lb} . \quad .60 ; \frac{1}{2} \mathrm{lb} .1 .00 ; 1 \mathrm{~b} .2 .00$ EDWARD'S PEREECTO ............ per pkt. $10 \mathrm{c} ;$ oz. $25 \mathrm{c} ; \frac{1}{4} \mathrm{lb} . \quad .80 ; \frac{1}{2} \mathrm{lb} .1 .50 ; 1 \mathrm{lb} .3 .00$ ROCKY FORD ................ per pkt. $5 \mathrm{c} ;$ oz. $15 \mathrm{c} ; \frac{1}{4} \mathrm{lb} . \quad .60 ; \frac{1}{2} \mathrm{lb} .1 .00 ; 1 \mathrm{~b} .2 .00$ DELICIOUS (Gold Lined) ........... per pkt. $10 \mathrm{c} ;$ oz. $25 \mathrm{c} ; \frac{1}{4} \mathrm{lb} . \quad .80 ; \frac{1}{2} \mathrm{lb} .1 .50 ; 1 \mathrm{lb} .3 .00$

\section{WATER MELUN}

HARRIS EARLY ................. per pkt. 5c; oz. $10 \mathrm{c} ; \frac{1}{4} \mathrm{lb} .30 \mathrm{c} ; \frac{1}{2} \mathrm{lb} .60 \mathrm{c} ; 1 \mathrm{lb} . \$ 1.00$ COLE'S EARLY . . . . . . . . . . . . . . . . per pkt. $5 \mathrm{c} ; \quad$ oz. $10 \mathrm{c} ; \frac{1}{4} \mathrm{lb} .30 \mathrm{c} ; \frac{1}{2} \mathrm{lb} .50 \mathrm{c} ; \mathrm{lb} .90$ IINTE CRISTO OR KECKLEY'S SWEET. . . per pkt. $5 \mathrm{c} ; \quad$ oz. $10 \mathrm{c} ; \frac{1}{4} \mathrm{lb} .35 \mathrm{c} ; \frac{1}{2} \mathrm{lb} .70 \mathrm{c} ; 1 \mathrm{~b} .1 .25$ SWEETHEART. ................ per pkt. $5 \mathrm{c} ; \quad$ oz. $10 \mathrm{c} ; \frac{1}{4} \mathrm{lb} .30 \mathrm{c} ; \frac{1}{2} \mathrm{lb} .60 \mathrm{c} ; \mathrm{lb} .1 .00$ HALBERT HONEY $\ldots \ldots \ldots \ldots \ldots \ldots \ldots \ldots$ perpkt. $5 \mathrm{c} ; \quad$ oz. $10 \mathrm{c} ; \frac{1}{4} \mathrm{lb} .30 \mathrm{c} ; \frac{1}{2} \mathrm{lb} .60 \mathrm{c} ; \mathrm{lb} .1 .00$

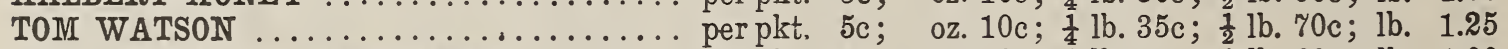
CITRON, Red and Green Seeded.......... per pkt. $5 \mathrm{c} ; \quad$ oz. $10 \mathrm{c} ; \frac{1}{4} \mathrm{lb} .30 \mathrm{c} ; \frac{1}{2} \mathrm{lb} .60 \mathrm{c} ; \mathrm{lb} .1 .00$ MUSTARD WHITE LONDON.................... pkt. 5c; oz. 15c; $\frac{1}{4}$ lb. 25c; lb. 70c

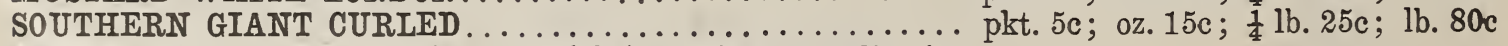
MUSHROOM SPAWN (English, in Bricks). Price on application. 


\section{ONION}

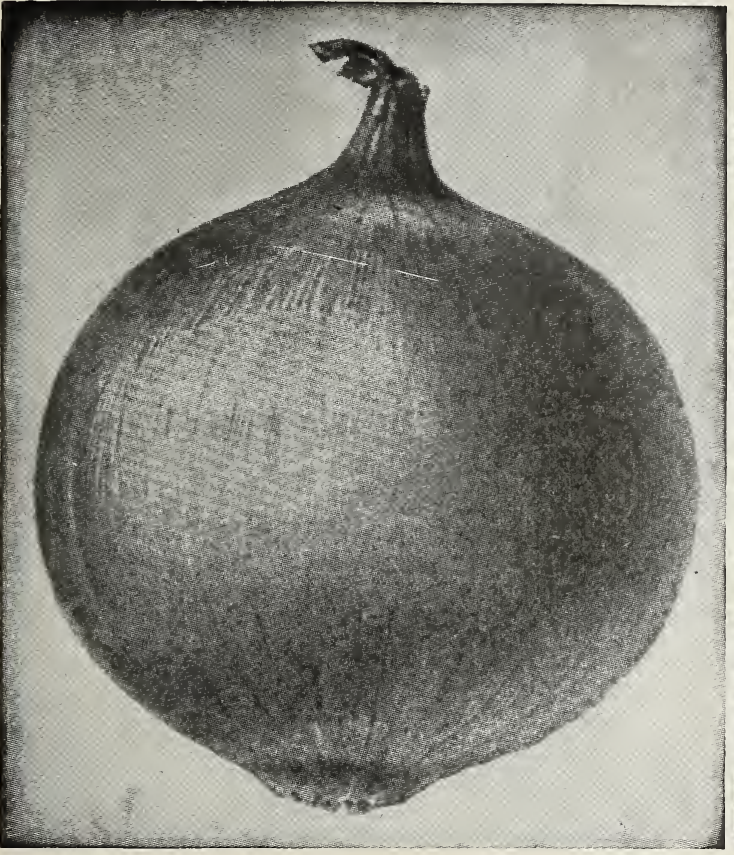

SOUTHPORT LARGE WHITE GLOBE

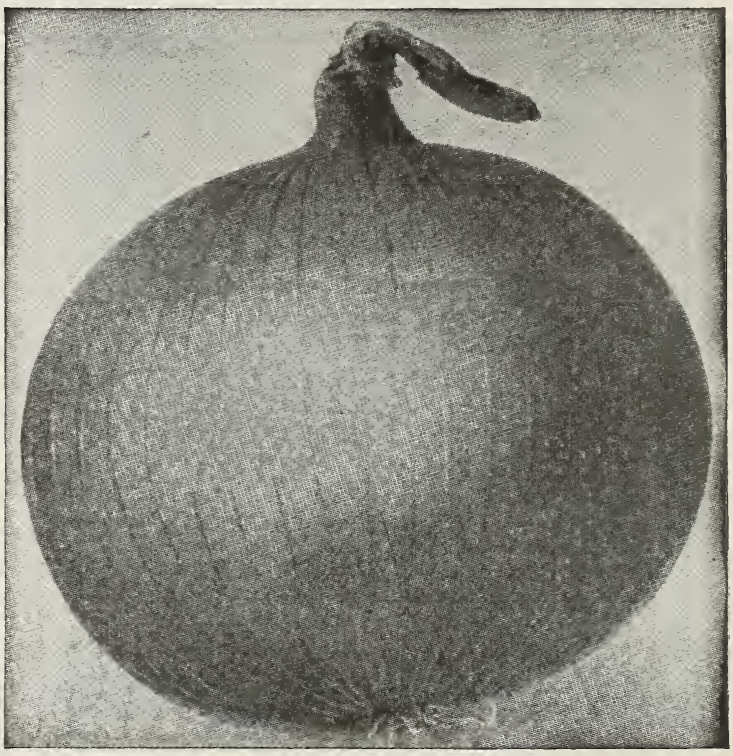

PRICE'S IMPROVED YELLOW GLOBE DANVERS

PRICE'S IMPROVED YELLOW GLOBE DANVERS. A special selected strain sold only by us. This variety is a great yielder and a fine keeper, and will produce more large, fine shaped, marketable Onions from an acre than any other variety of Yellow Globe Danvers.

pkt. $5 \mathrm{c} ; \frac{1}{2}$ oz. $15 \mathrm{c} ;$ oz. $25 \mathrm{c} ; \frac{1}{4}$ lb. $\$ 0.80 ; \frac{1}{2}$ lb. $\$ 1.25 ;$ lb. $\$ 2.00$ SOUTHPORT YELLOW GLOBE.. pkt. $5 \mathrm{c} ; \frac{1}{2}$ oz. $10 \mathrm{c} ;$ oz. $20 \mathrm{c} ; \frac{1}{4} \mathrm{lb} . \quad .60 ; \frac{1}{2} \mathrm{lb} .1 .00 ; \mathrm{lb} .1 .90$ MICHIGAN YELLOW GLOBE... pkt. $5 \mathrm{c} ; \frac{1}{2}$ oz. $10 \mathrm{c} ; 0 \mathrm{oz} .20 \mathrm{c} ; \frac{1}{4} \mathrm{lb} . \quad .60 ; \frac{1}{2} \mathrm{lb} .1 .00 ; 1 \mathrm{~b} .1 .90$ OHIO YELLOW GLOBE........ pkt. $5 \mathrm{c} ; \frac{1}{2}$ oz. $10 \mathrm{c} ;$ oz. $20 \mathrm{c} ; \frac{1}{4} \mathrm{lb} . \quad .60 ; \frac{1}{2} \mathrm{lb} .1 .00 ; 1 \mathrm{~b} .1 .90$ MAMMOTH PRIZETAKER ..... pkt. $5 \mathrm{c} ; \frac{1}{2}$ oz. $15 \mathrm{c} ;$ oz. $25 \mathrm{c} ; \frac{1}{4} \mathrm{lb} . \quad .80 ; \frac{1}{2} \mathrm{lb} .1 .25 ; 1 \mathrm{~b} .2 .00$

\section{WHITE VARIETIES}

SOUTHPORT WHITE GLOBE.... pkt. $5 \mathrm{c} ; \frac{1}{2}$ oz. $10 \mathrm{c} ;$ oz. $20 \mathrm{c} ; \frac{1}{4}$ lb. $70 \mathrm{c} ; \frac{1}{2} \mathrm{lb} . \$ 1.25 ; 1 \mathrm{lb} . \$ 2.50$ PORTUGAL OR SILVER SKUN. . pkt. $5 \mathrm{c} ; \frac{1}{2}$ oz. $10 \mathrm{c} ; \mathrm{oz} .20 \mathrm{c} ; \frac{1}{4} \mathrm{lb} .70 \mathrm{c} ; \frac{1}{2} \mathrm{lb} .11 .25 ; 1 \mathrm{~b} .2 .50$ EARLY WHITE PEARL....... pkt. $5 \mathrm{c} ; \frac{1}{2} \mathrm{oz} .15 \mathrm{c} ;$ oz. $25 \mathrm{c} ; \frac{1}{4} \mathrm{lb} .80 \mathrm{c} ; \frac{1}{2} \mathrm{lb} .1 .40 ; 1 \mathrm{~b} .2 .50$ WHITE QUEEN ........... pkt. $5 \mathrm{c} ; \frac{1}{2}$ oz. $15 \mathrm{c} ;$ oz. $25 \mathrm{c} ; \frac{1}{4} \mathrm{lb} .90 \mathrm{c} ; \frac{1}{2} \mathrm{lb} .1 .60 ; 1 \mathrm{~b} .3 .00$ WHITE BARLETTA ......... pkt. $5 \mathrm{c} ; \frac{1}{2}$ oz. $15 \mathrm{c} ;$ oz. $25 \mathrm{c} ; \frac{1}{4}$ lb. $90 \mathrm{c} ; \frac{1}{2}$ lb. $1.60 ; 1 \mathrm{lb} .3 .00$

\section{RED VARIETIES}

SOUTHPORT RED GLOBE...... pkt. $5 \mathrm{c} ; \frac{1}{2}$ oz. $15 \mathrm{c} ;$ oz. $20 \mathrm{c} ; \frac{1}{4}$ lb. $70 \mathrm{c} ; \frac{1}{2}$ lb. $\$ 1.35 ; 1 \mathrm{lb} . \$ 2.50$ RED WETHERSFIELD ....... pkt. $5 \mathrm{c} ; \frac{1}{2}$ oz. $10 \mathrm{c} ; 0 \mathrm{oz} .20 \mathrm{c} ; \frac{1}{4} \mathrm{lb} .70 \mathrm{c} ; \frac{1}{2} \mathrm{lb} .1 .25 ; 1 \mathrm{lb} .2 .00$ EARLY RED FLAT......... pkt. $5 \mathrm{c} ; \frac{1}{2}$ oz. $15 \mathrm{c} ;$ oz. $25 \mathrm{c} ; \frac{1}{4} \mathrm{lb} .60 \mathrm{c} ; \frac{1}{2} \mathrm{lb} .1,25 ; 1 \mathrm{~b} .2 .00$

ONION SETS, WHITE, YELLOW, RED AND YELLOW MULTIPLIERS. 


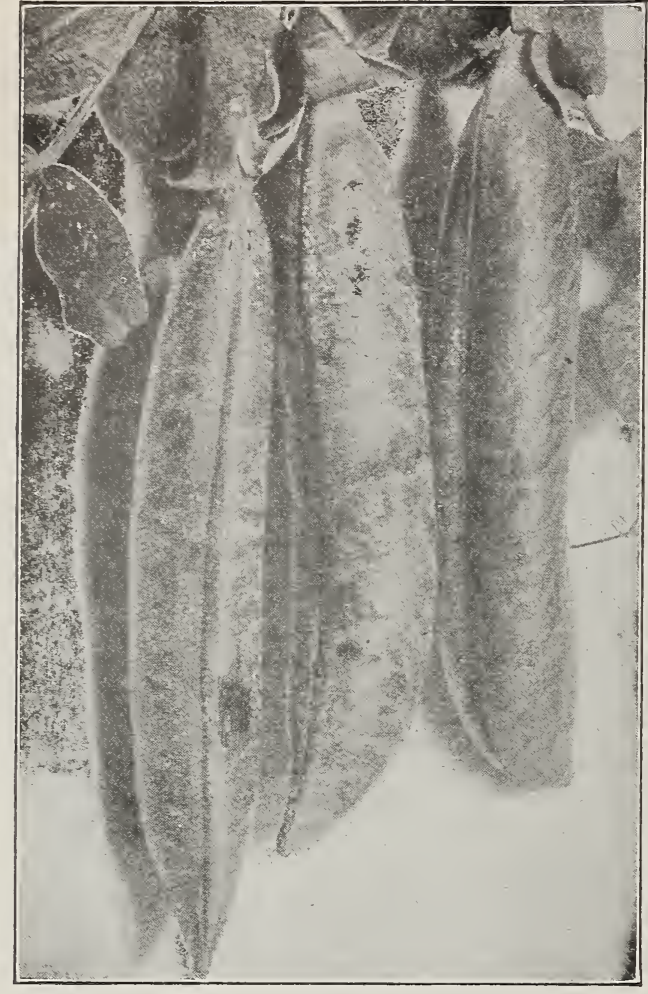

GRADUS OR PROSPERITY

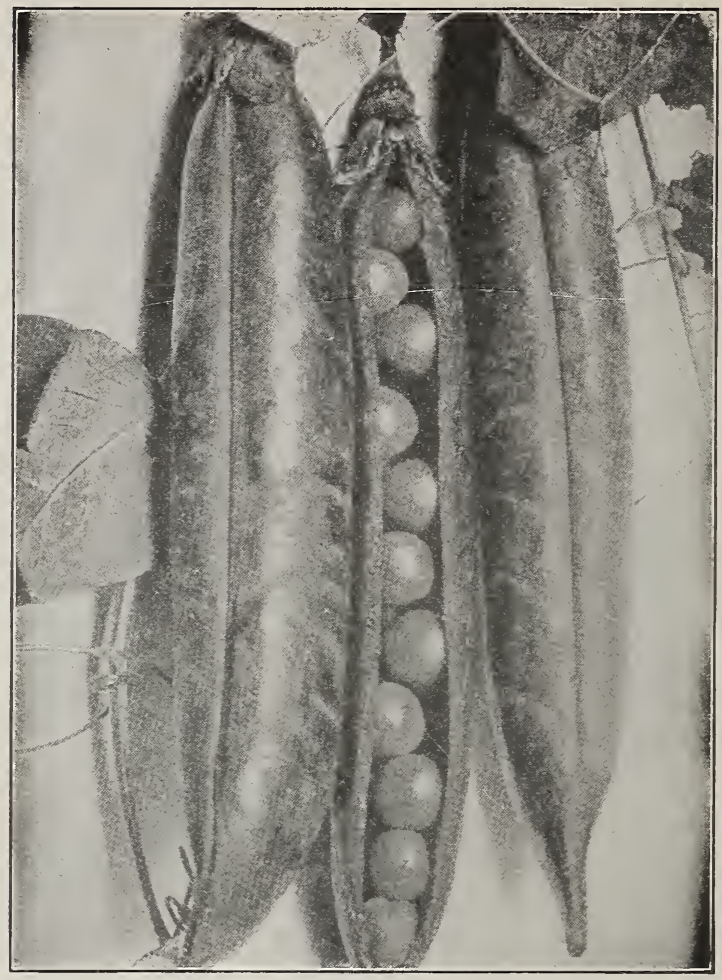

PRICE'S IMPROVED TELEPHONE

\section{PEAS}

PACKETS OF ALL VARIETIES, $10 \mathrm{c}$.

\section{EXTRA EARLY SMOOTH VARIETIES}

PRICE'S EARLIEST ................ $\frac{1}{1}$ lb. $15 \mathrm{c} ; \frac{1}{2} \mathrm{lb} .20 \mathrm{c} ; \mathrm{lb} .30 \mathrm{c} ; 2 \mathrm{lbs} .50 \mathrm{c} ; 5$ lbs. $\$ 1.20$ PRICE'S LIGHTNING ................ $\frac{1}{2} \mathrm{lb} .15 \mathrm{c} ; \frac{1}{2} \mathrm{lb} .20 \mathrm{c} ; \mathrm{lb} .30 \mathrm{c} ; 2 \mathrm{lbs} .50 \mathrm{c} ; 5$ lbs. 1.20 PRICE'S PROLIFIC EARLY MARKET..... $\frac{1}{1} \mathrm{lb} .15 \mathrm{c} ; \frac{1}{2} \mathrm{lb} .20 \mathrm{c}$; lb. $30 \mathrm{c} ; 2 \mathrm{lbs} .50 \mathrm{c} ; 5 \mathrm{lbs} .1 .20$ FIRST OF ALL................... $1 \mathrm{lb} .15 \mathrm{c} ; \frac{1}{2} \mathrm{lb} .20 \mathrm{c} ; \mathrm{lb} .30 \mathrm{c} ; 2 \mathrm{lbs} .50 \mathrm{c} ; 5 \mathrm{lbs} .1 .20$ ALASKA. ........................ $\frac{1}{4}$ lb. $15 \mathrm{c} ; \frac{1}{2} \mathrm{lb} .20 \mathrm{c} ; 1 \mathrm{lb} .25 \mathrm{c} ; 2$ lbs. $45 \mathrm{c} ; 5$ lbs. 1.10 EARLIEST OF ALL................. $\frac{1}{16} .15 \mathrm{c} ; \frac{1}{2} \mathrm{lb} .20 \mathrm{c} ; 1 \mathrm{lb} .25 \mathrm{c} ; 2 \mathrm{lbs} .45 \mathrm{c} ; 5$ lbs. 1.10 AMEER. . . . ................... $\frac{1}{4}$ lb. $15 \mathrm{c} ; \frac{1}{2} \mathrm{lb} .20 \mathrm{c} ; 1 \mathrm{lb} .30 \mathrm{c} ; 2 \mathrm{lbs} .50 \mathrm{c} ; 5$ lbs. 1.20 ALASKA (large podded) ............... $\frac{1}{1} \mathrm{lb} .15 \mathrm{c} ; \frac{1}{2} \mathrm{lb} .20 \mathrm{c} ; 1 \mathrm{lb} .25 \mathrm{c} ; 2 \mathrm{lbs} .45 \mathrm{c} ; 5$ lbs. 1.10

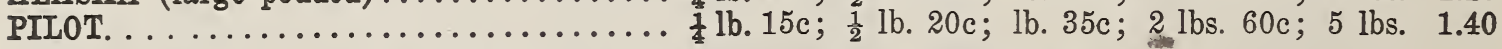

\section{EARLY WRINKLED VARIETIES}

LITTLE MIARVEL ................. $\frac{1}{1} \mathrm{lb} .15 \mathrm{c} ; \frac{1}{2} \mathrm{lb} .20 \mathrm{c} ; \mathrm{lb} .35 \mathrm{c} ; 2 \mathrm{lbs} .60 \mathrm{c} ; 5 \mathrm{lbs} . \$ 1.40$ LAXTONIAN. . ................... $\frac{1}{4} \mathrm{lb} .15 \mathrm{c} ; \frac{1}{2} \mathrm{lb} .20 \mathrm{c} ; \mathrm{lb} .35 \mathrm{c} ; 2 \mathrm{lbs} .60 \mathrm{c} ; 5 \mathrm{lbs} .1 .40$ EARLY IMORN .................... $\frac{1}{2} \mathrm{lb} .15 \mathrm{c} ; \frac{1}{2} \mathrm{lb} .20 \mathrm{c} ; \mathrm{lb} .35 \mathrm{c} ; 2 \mathrm{lbs} .60 \mathrm{c} ; 5$ lbs. 1.40 GRADUS. ........................ $1 \mathrm{lb} .15 \mathrm{c} ; \frac{1}{2} \mathrm{lb} .20 \mathrm{c} ; \mathrm{lb} .35 \mathrm{c} ; 2 \mathrm{lbs} .60 \mathrm{c} ; 5 \mathrm{lbs} .1 .40$ THOMAS LAXTON ................. $\frac{1}{4} \mathrm{lb} .15 \mathrm{c} ; \frac{1}{2} \mathrm{lb} .20 \mathrm{c} ; \mathrm{lb} .35 \mathrm{c} ; 2 \mathrm{lbs} .60 \mathrm{c} ; 5 \mathrm{lbs} .1 .40$ BLUE BANTAM .................... $1 \mathrm{lb} .20 \mathrm{c} ; \frac{1}{2} \mathrm{lb} .25 \mathrm{c} ; \mathrm{lb} .40 \mathrm{c} ; 2 \mathrm{lbs} .70 \mathrm{c} ; 5 \mathrm{lbs} .1 .50$ PRICE'S EARLY DWARF............... $\frac{1}{4} \mathrm{lb} .15 \mathrm{c} ; \frac{1}{2} \mathrm{lb} .20 \mathrm{c} ; \mathrm{lb} .35 \mathrm{c} ; 2 \mathrm{lbs} .60 \mathrm{c} ; 5 \mathrm{lbs} .1 .40$ SURPRISE. . . .................... $\frac{1}{16}$ lb. $20 \mathrm{c} ; \frac{1}{2} \mathrm{lb} .25 \mathrm{c} ; \mathrm{lb} .40 \mathrm{c} ; 2$ lbs. $70 \mathrm{c} ; 5$ lbs. 1.50 SUTTON'S PIONEER ................ $\frac{1}{1} \mathrm{lb} .15 \mathrm{c} ; \frac{1}{2} \mathrm{lb} .20 \mathrm{c} ; \mathrm{lb} .35 \mathrm{c} ; 2 \mathrm{lbs} .60 \mathrm{c} ; 5$ lbs. 1.40 
PEAS (Continued)

SECOND EARLY AND MAIN CROP (Wrinkled Varieties)

EARLY PREMIUUM GEM................ $\frac{1}{4}$ lb. $15 \mathrm{c} ; \frac{1}{2} \mathrm{lb} .20 \mathrm{c} ; \mathrm{lb} .30 \mathrm{c} ; 2$ lbs. $50 \mathrm{c} ; 5$ lbs. $\$ 1.20$

AMERICAN WONDER

$\frac{1}{4}$ lb. $15 \mathrm{c} ; \frac{1}{2}$ lb. $20 \mathrm{c} ; 1 \mathrm{~b} .30 \mathrm{c} ; 2$ lbs. $50 \mathrm{c} ; 5$ lbs. 1.20

NOTT'S EXCELSIOR .

$\frac{1}{4}$ lb. $15 \mathrm{c} ; \frac{1}{2}$ lb. $20 \mathrm{c} ; 1 \mathrm{~b} .30 \mathrm{c} ; 2$ lbs. $50 \mathrm{c} ; 5$ lbs. 1.20

SUTTON'S EXCELSIOR

POTLATCH.

$\frac{1}{4} \mathrm{lb} .15 \mathrm{c} ; \frac{1}{2} \mathrm{lb} .20 \mathrm{c} ; \mathrm{lb} .30 \mathrm{c} ; 2 \mathrm{lbs} .50 \mathrm{c} ; 5 \mathrm{lbs} .1 .20$

BLISS EVERBEARTNG

STRATAGEM . . .

$\frac{1}{4} \mathrm{lb} .15 \mathrm{c}$;

$\frac{1}{4} 1 \mathrm{~b} .15 \mathrm{c}$;

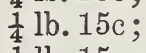

lb. $20 \mathrm{c} ; 1$ lb. $35 \mathrm{c} ; 2$ lbs. $60 \mathrm{c} ; 5$ lbs. 1.40

ALDERIMAN.

$\frac{1}{4} \mathrm{lb} .15 \mathrm{c}$;

lb. 20c; lb. 30c; 2 lbs. 50c; 5 lbs.

1.20

PRINCE EDWARD

$\frac{1}{4} 1 \mathrm{~b} .15 \mathrm{c}$;

lb. $20 \mathrm{c} ; 1 \mathrm{lb} .30 \mathrm{c} ; 2$ lbs. $50 \mathrm{c} ; 5$ lbs.

1.20

DUKE OF ALBANY

PRICE'S TELEPHONE ............... $\frac{1}{4} 1 \mathrm{lb} .15 \mathrm{c} ; \frac{1}{2} \mathrm{lb} .20 \mathrm{c} ; 1 \mathrm{~b} .30 \mathrm{c} ; 2$ lbs. $50 \mathrm{c} ; 5$ lbs. 1.20

$\frac{1}{4} \mathrm{lb} .15 \mathrm{c}$;

lb. $20 \mathrm{c} ; \mathrm{lb} .35 \mathrm{c} ; 2$ lbs. $60 \mathrm{c} ; 5 \mathrm{lbs}$.

1.40

$\frac{1}{2}$ lb. $20 \mathrm{c} ; 1 \mathrm{~b} .30 \mathrm{c} ; 2$ lbs. $50 \mathrm{c} ; 5$ lbs. 1.20

LONG ISLAND MAMIMOTH............. $\frac{1}{4} \mathrm{lb} .15 \mathrm{c} ; \frac{1}{2} \mathrm{lb} .20 \mathrm{c} ; \mathrm{lb} .35 \mathrm{c} ; 2 \mathrm{lbs} .60 \mathrm{c} ; 5 \mathrm{lbs} .1 .40$

DWARF CHAMIPION OR JUNO........... $\frac{1}{4} \mathrm{lb} .15 \mathrm{c} ; \frac{1}{2} \mathrm{lb} .20 \mathrm{c} ; 1 \mathrm{~b} .30 \mathrm{c} ; 2 \mathrm{lbs} .50 \mathrm{c} ; 5 \mathrm{lbs} .1 .20$

CHAIIPION OF ENGLAND............. $\frac{1}{4} \mathrm{lb} .15 \mathrm{c} ; \frac{1}{2} \mathrm{lb} .20 \mathrm{c} ; 1 \mathrm{~b} .30 \mathrm{c} ; 2 \mathrm{lbs} .50 \mathrm{c} ; 5 \mathrm{lbs} .1 .20$

DWARF TELEPHONE ............... $\frac{1}{4} \mathrm{lb} .15 \mathrm{c} ; \frac{1}{2} \mathrm{lb} .20 \mathrm{c} ; \mathrm{lb} .35 \mathrm{c} ; 2 \mathrm{lbs} .60 \mathrm{c} ; 5$ lbs. 1.40

\section{EDIBLE POD VARIETIES}

DWARF GRAY SUGAR.............. $\frac{1}{4} \mathrm{lb} .15 \mathrm{c} ; \frac{1}{2} \mathrm{lb} .25 \mathrm{c} ; \mathrm{lb} .40 \mathrm{c} ; 2 \mathrm{lbs} . \$ 0.75 ; 5$ lbs. $\$ 1.75$ LUSCIOUS SUGAR ................ $\frac{1}{4} \mathrm{lb} .15 \mathrm{c} ; \frac{1}{2} \mathrm{lb} .25 \mathrm{c} ; \mathrm{lb} .40 \mathrm{c} ; 2 \mathrm{lbs} . \quad .75 ; 5$ lbs. 1.75

\section{PARSLEY}

EXTRA DOUBLE CURLED.

MIOSS CURLED

HAMIBURG (or rooted)

PLAIN. per pkt. $5 \mathrm{c} ; \frac{1}{2}$ oz. $10 \mathrm{c} ;$ oz. $15 \mathrm{c} ; \frac{1}{4}$ lb. $40 \mathrm{c}$ per pkt. $5 \mathrm{c} ; \frac{1}{2}$ oz. $10 \mathrm{c} ;$ oz. $15 \mathrm{c} ; \frac{1}{4}$ lb. $40 \mathrm{c}$ per pkt. $5 \mathrm{c} ; \frac{1}{2}$ oz. $10 \mathrm{c} ;$ oz. $15 \mathrm{c} ; \frac{1}{4}$ lb. $35 \mathrm{c}$ per pkt. $5 \mathrm{c} ; \frac{1}{2}$ oz. $10 \mathrm{c} ;$ oz. $15 \mathrm{c} ; \frac{1}{4} \mathrm{lb} .35 \mathrm{c}$

\section{PARSNIP}

PRICE'S IDEAL HOLLOW CROWN........ per pkt. 10c; oz. 20c; $\frac{1}{4} \mathrm{lb} .40 \mathrm{c} ; \frac{1}{2} \mathrm{lb} .70 \mathrm{c} ; 1 \mathrm{lb} .90 \mathrm{c}$ SUTTON'S STUDENT

LONG SMOOTH

per pkt. $10 \mathrm{c} ;$ oz. $20 \mathrm{c} ; \frac{1}{4}$ lb. $40 \mathrm{c} ; \frac{1}{2}$ lb. $70 \mathrm{c} ; \mathrm{lb} .90 \mathrm{c}$ per pkt. $10 \mathrm{c} ;$ oz. $20 \mathrm{c} ; \frac{1}{4}$ lb. $40 \mathrm{c} ; \frac{1}{2}$ lb. $70 \mathrm{c} ; \mathrm{lb} .90 \mathrm{c}$

\section{PEPPER}

PRICE'S GIANT SWEET................... per pkt. $10 \mathrm{c} ; \frac{1}{2}$ oz. $35 \mathrm{c} ;$ oz. $60 \mathrm{c} ; \frac{1}{4} \mathrm{lb} . \$ 2.25$ CRIMISON GIANT ......................... per pkt. $10 \mathrm{c} ; \frac{1}{2} \mathrm{oz} .35 \mathrm{c} ; \mathrm{oz} .60 \mathrm{c} ; \frac{1}{1} \mathrm{lb} .2 .25$ IMPROVED RUBY KING................... per pkt. $10 \mathrm{c} ; \frac{1}{2}$ oz. $30 \mathrm{c} ;$ oz. $50 \mathrm{c} ; \frac{1}{4} \mathrm{lb} .1 .90$ BELL OR BULL NOSE..................... per pkt. $10 \mathrm{c} ; \frac{1}{2}$ oz. $30 \mathrm{c} ;$ oz. $50 \mathrm{c} ; \frac{1}{4} \mathrm{lb} .1 .90$ WORLD BEATER ......................... per pkt. $10 \mathrm{c} ; \frac{1}{2}$ oz. $30 \mathrm{c} ;$ oz. $50 \mathrm{c} ; \frac{1}{4} \mathrm{lb} .1 .90$ PIMENTO (Sweet Salad) .................... per pkt. 10c; $\frac{1}{2}$ oz. $30 \mathrm{c} ;$ oz. $50 \mathrm{c} ; \frac{1}{4} \mathrm{lb} .1 .90$ PERFECTION. . . ...................... per pkt. $10 \mathrm{c} ; \frac{1}{2}$ oz. $30 \mathrm{c}$; oz. $50 \mathrm{c} ; \frac{1}{4}$ lb. 2.00 LONG RED CAYENNE (for Pickles)............. per pkt. $10 \mathrm{c} ; \frac{1}{2}$ oz. $30 \mathrm{c} ;$ oz. $50 \mathrm{c} ; \frac{1}{4} \mathrm{lb} .2 .0 \mathrm{c}$

\section{PUMPKIN}

LARGE CHEESE per pkt. 5c; oz. 10c; $\frac{1}{4}$ lb. 30c; SUGAR OR NEW ENGLAND PIE.

CUSHAW (Striped) GOLDEN OBLONG CONNECTICUT FIELD WINTER LUXURY MIAMIMOTH KING (Large) per pkt. $5 \mathrm{c} ;$ oz. $10 \mathrm{c} ; \frac{1}{4}$ lb. $30 \mathrm{c}$; per pkt. $5 \mathrm{c} ; 0 z .15 \mathrm{c} ; \frac{1}{4} \mathrm{lb} .35 \mathrm{c}$; per pkt. $5 \mathrm{c}$; oz. $10 \mathrm{c} ; \frac{1}{4} 1 \mathrm{lb} .25 \mathrm{c}$; per pkt. $5 \mathrm{c}$; oz. $10 \mathrm{c}$; $\frac{1}{4} \mathrm{lb} .25 \mathrm{c}$; per pkt. $5 \mathrm{c}$; oz. $15 \mathrm{c} ; \frac{1}{4} \mathrm{lb} .35 \mathrm{c}$; $\frac{1}{2}$ lb. $60 \mathrm{c} ; \mathrm{lb} . \$ 1.00$ $\frac{1}{2} \mathrm{lb} .60 \mathrm{c} ; \mathrm{lb} .1 .00$ $\frac{1}{2} \mathrm{lb} .60 \mathrm{c} ; \mathrm{lb} .1 .10$ $\frac{1}{2} \mathrm{lb} .50 \mathrm{c} ; \mathrm{lb} . \quad .90$ $\frac{1}{2}$ lb. $45 \mathrm{c} ; \mathrm{lb} . \quad 80$ per pkt. $5 \mathrm{c} ;$ oz. $15 \mathrm{c}$; $\frac{1}{4} \mathrm{lb} .35 \mathrm{c}$;

$\frac{1}{2} \mathrm{lb} .60 \mathrm{c} ; \mathrm{lb} .1 .10$ $\frac{1}{2} \mathrm{lb} .60 \mathrm{c} ; \mathrm{lb} .1 .10$ 


\section{MAINE SEED POTATOES}

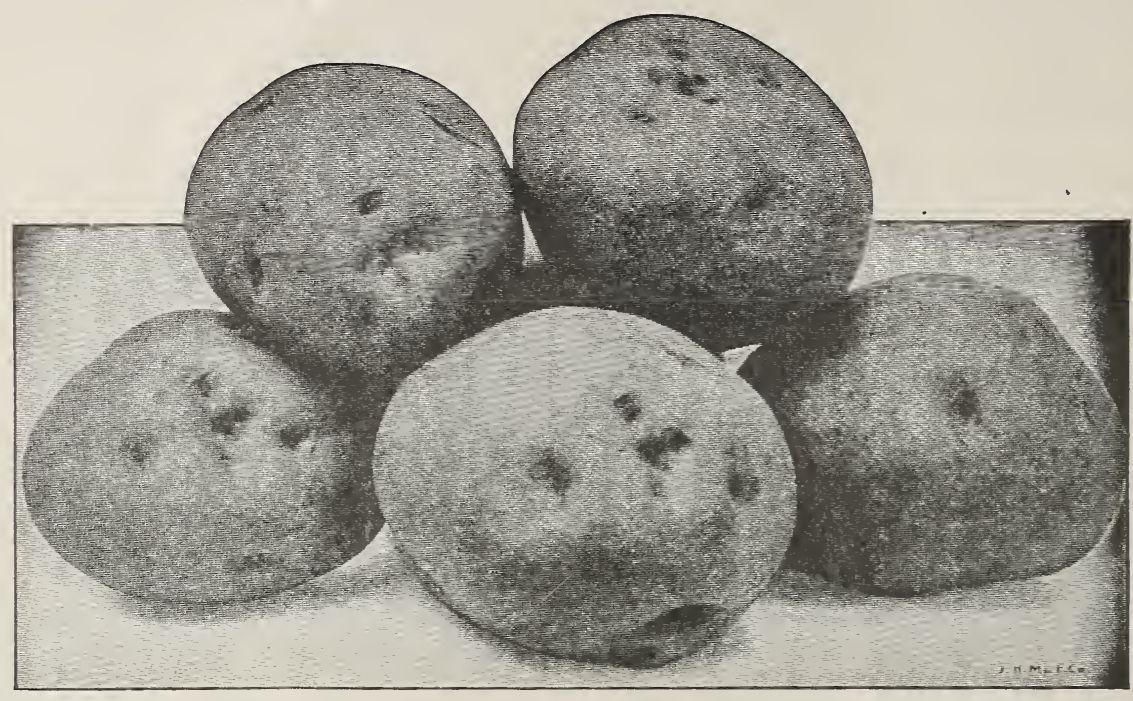

IKISH CUBBLER POTATOES

Prices Subject to Variations of the Market.

Barrels and Sacks contain 165 lbs. of Potatoes.

Price on application.

A good sandy loam produces the best potatoes, but they can be grown on all kinds of soil. New or pasture land, with the turf freshly turned, produces the finest crop. Make furrows of good depth, three feet apart. Scatter a liberal dressing of phosphate or decayed stable manure along the drill and set the seed about ten inches apart in the rows. Cover with about two inches of soil, and begin to cultivate when the plants are well up. At each successive hoeing bring additional soil about the plants. Four barrels are sufficient to plant one acre in drills.

These potatoes are grown especially for seed, in Maine, and are entirely free from rot or powdery cab. They are true to name, clean and smooth, and will be very desirable to plant, as they will insure earlier and better sized crops than could be had from any grown about here. Changing your seed will improve jour crop and prodice a much better quality and larger vield. In this way you will be more than repaid for the money expended.

\section{EARLY VARIETIES}

OHI0. Round to oblong in shape, pink skin, white flesh, very early.

EARLY SIX WEEKS. Oblong in shape, pink skin, white flesh, early.

IRISH COBBLER. The flesh is white, an excellent cooker, makes a good general crop potato and yields well on mast any soil.

PRICE'S EARIY SUNRISE. Tubers are oblong in shape, very uniform, white flesh, an excellent early variety for family use.

EARLY ROSE. Shape long and good size. It ccoks mealy and is of good flavor.

EARLY MANISTEE. Round to oblong in shape, slightly flattened, skin light pink, flesh pure white, cooks well and yields well on sandy soil.

\section{MEDIUIM EARIY AND LATE VARIETIES}

BOVEE. An excellent cooker, flesh white, and a heavy yielder.

NEW QUEEN. Fine large size potato, resembling the Hebron in shape and color. GOID COIN. A late round white potato, good cooker, and a good winter variety. MONEYMAKER. Very productive, shape long, color white, an excellent baking potato.

CARMEN NO. 3. Large round white, main crop and a good yielder.

GREEN MOUNTATN. White skin, size uniform, does best in light and loamy soil. UNCLE SAM. A main crop variety, oval shaped, flesh white, light russet skin.

RUSSET. A very fine main crop potato, round to oblong tubers, pure white flesh, russet skin. AMERICAN GIANT. Late variety, shape long, flesh white, a good late cropper. 


\section{RADISH}

Sow early varieties in the Spring, as early as the ground can be worked, in drills ten inches apart, covering the seed hall an inch anes

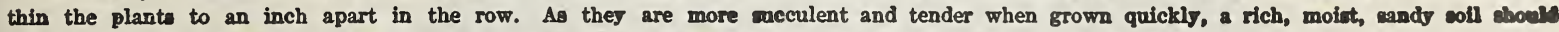
be nelected, and frequently watered in dry weather. For a succenion sow every two weeks till midsummer.

\section{Packets, 5 cents.}

$\operatorname{Pr}$ oz $1 / 4, \mathrm{lb}$

PRICE'S FORCING. Beautiful red, very early.... $\$ 015 \$ 050$ FXTRA EARLY SCARLET TURNIP. In hape and form like Scarlet Turnip, but is earlier and has

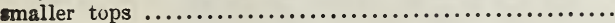

EXTRA EARLY DEEP SCARLET TURNIP

NON PLUS ULTRA. Round in shape; deep red color; short top; tender and fine flavor; very early........

ROSY GEM or RAPID FORCING. An early variety, round in shape; crisp and tender...............

EARLY SCARLET GLOBE. A fine variety for forcing. Shape roundish oval; skin red; flesh white, solid

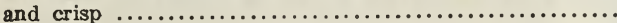

EXTRA EARLY RED FORCING. Turnip shape; short top, dark red; white flesh. Fine for forcing........

EARLY SCARLET TURNIP. Standard variety, mall, round, red, turnip-shaped, mild and crisp......... FARLY DEEP SCARLET TURNIP. Quick growing, mild, bright color, good shape, tender......... EXTRA EARLY WHITE TURNIP. Rapid

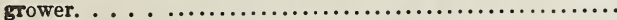

SCARLET TURNIP, WHITE TIP. An early variety, red top and white bottom..................... OLIVE-SHAPED SCARLET. Good quality...... OLIVE-SHAPED DEEP SCARLET. Deę

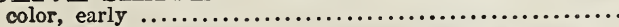
Olive-Shaped Scarlet, IVhite Tip. Forcing....
Olive-Shaped White.

Giant Stuttgart. Of a large oize and early. Flesh and skin pure white; quality fine; firm and brittle...... WHITE STRASBURG. Of a tapering shape. Skin and flesh white. Fine Summer variety.................. Yellow Summer Turnip. Turnip-shaped, of gray or russet color, growing to a large size...................... GOLDEN YELLOW OVAL................... ICICLE. Finest long, white, tender variety........... BLACK SPANISH WINTER, LONG. Very hardy and fine for winter use....................... Black Spanish Winter, Round. For Winter;

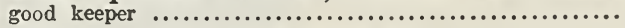
Half Long Black. Large and fine................ CHINESE ROSE, Winter. Color, bright rose; excellent flavor; good at all seasons...................... WHITE CELESTIAL. Best of the large white radishes, flesh crisp and mild...........................

$\operatorname{Pr}$ oz $1 / 6 \mathrm{lb}$ $\$ 015 \$ 040$

1540

$15 \quad 25$

$15 \quad 40$

$15 \quad 30$

15 40

1540

$15 \quad 25$

$15 \quad 40$

$15 \quad 83$

$15 \quad 85$

\section{RHUBARB.}

Sow in drills eighteen inches apart; cover one inch deep; this plants to six inches. In the fall trench a piece of ground and manume it well; transplant the young plants into it, three feet apart each way. Cover with litter the first winter; a dreasing of coarse manure should be given each fall. It is better to buy roots which yield at once. Linnaeus. Standard. Per packet, 5 centa; per ounce, 40 ceate. Roots. 15 cents; $\$ 1.50$ per doz; by mail, 20 cents each: $\$ 2.25$ per doz.

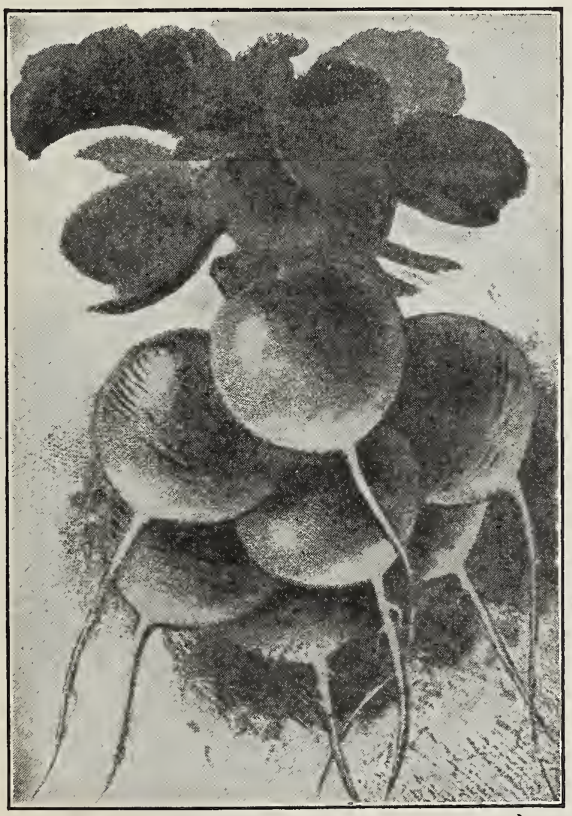

SOARLET TURNIP WHITE TIP RADISH.

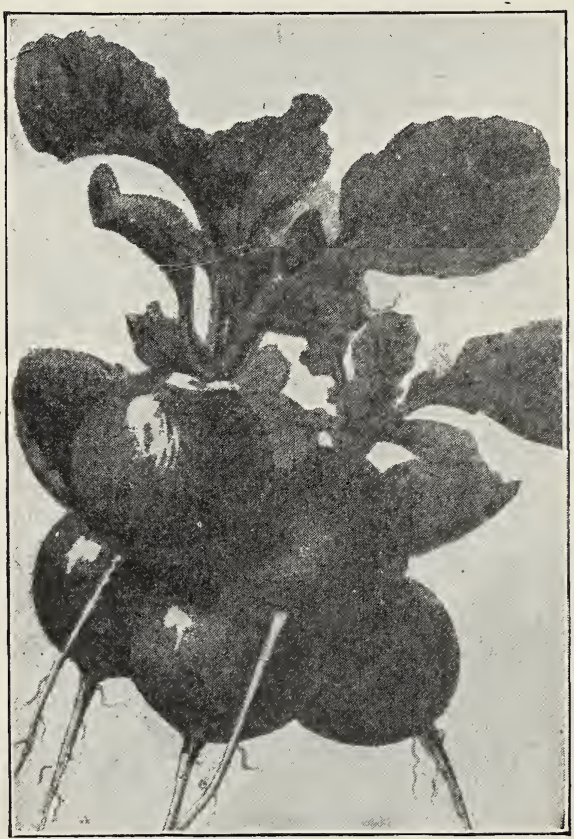

SCARLET GLOBE RADISH. 


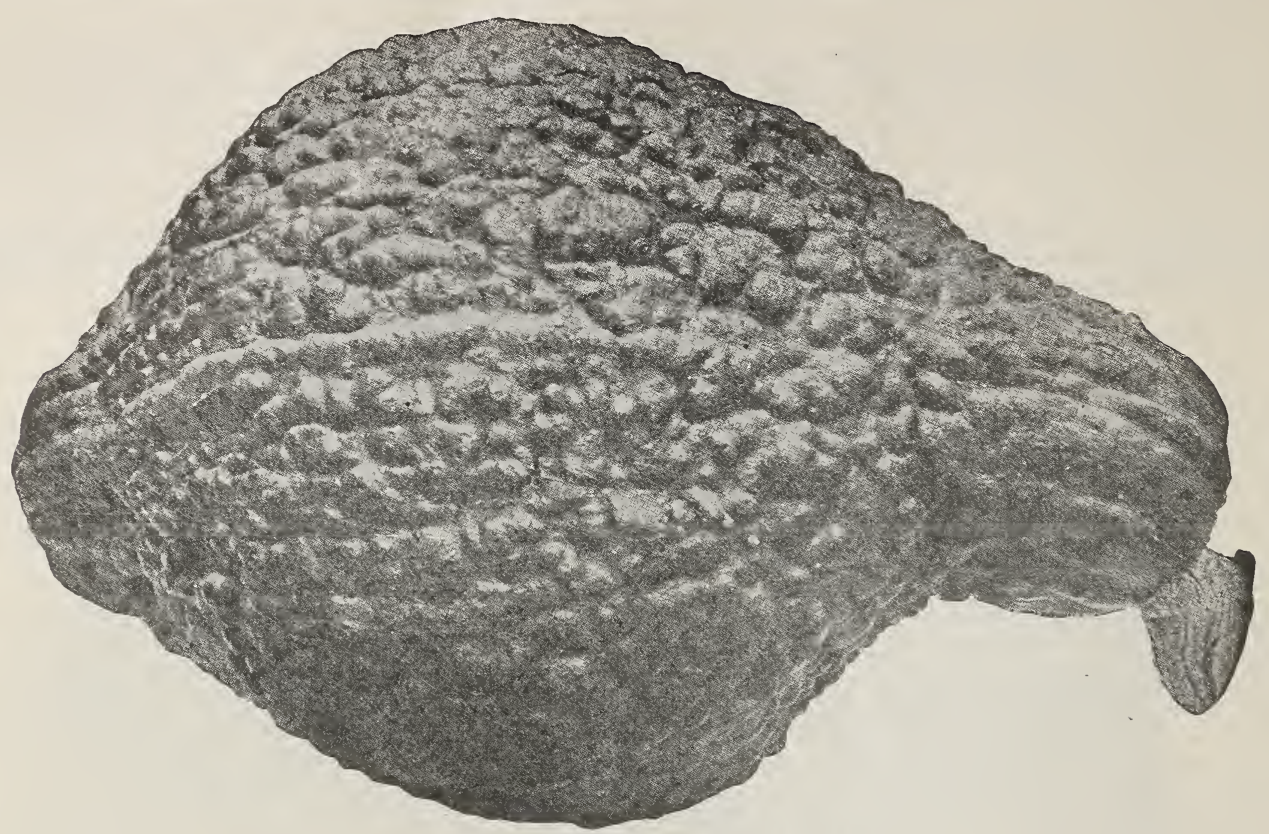

IMPROVED WARTED HUBBARD SQUASH

\section{SQUASH.}

CULTORE-Prepare the ground by thoroughly pulverizing it. Manure highly. All vines delight in warm and rich soil. Plant in hills 9 to 10 feet a part for running varieties, 5 to 6 feet for bush sort, working some fine, rich manure into each hill. Plant 8 or 10 seeds and when out of danger of bugs leave only two plants to the hill. Dust with lime or slug shot to protect from bugs in the early stages of growth. Cultivate till runners are well started.

\section{Packets of each variety, 5 cents.}

MAMMOTH WHITE BUSH. An improvement on the white scalloped bush variety, grow. ing double the size, ripens early, very productive. . . . ........................... \$ $\$ .30$

GIANT SUMMER CROOKNECK a very large variety of Summer crookneck, having a thick flesh and very few seeds........... 15

PROLIFIC ORANGE MARROW.

BOSTON MARROW. Color bright orange, flesh deep orange, fine flavored, a good

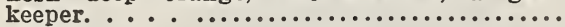

HUBBARD. The standard late variety, large size, fine flesh, close grained-dry and fine

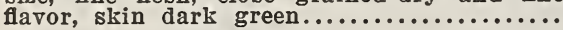

WARTED HUBBARD. Good quality resembling Hubbard, more warted skin...........

GOLDEN HUBBARD. In shape and size resembling Fubbard, but of rich orange red in color, and earlier....................

DELICIOUS. . . . ..................

GOLDEN CUSTARD.

FORDHOOK BUSH.

COCOZELLA OR ITALIAN MARROW......

VEGETABLE CREAM MARROW. A favorite English variety, skin greenish yellow, flesh

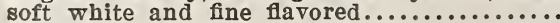

MAMMOTH CHILI. Grows to an enormous size, often weighing 150 lbs., color light

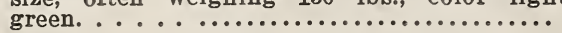

oz. $1 / 4$ Ib.

ROUND LEAF. Light green early maturing, variety best for early sowing............

LONG STANDING. A round leaf variety, standing from two to three weeks longer than other sorts, without going to seed, best for summer sowing.................

VICTORIA. Large thick dark green leaf, curled in center stands up well,..........

SAVOY LEAVED. Leaves thick large and fleshy, producing twice the weight of ordinary sorts, good for late summer or fall sow.

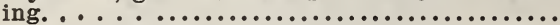

LONG SEASONS. A heavy crumpled leaved variety, dark green, excellent flavor........

PRICE'S CRUMPLE LEAF, A heavy dark green crumpled leaved variety excellent for the kitchen garden, can be sowed early or late. . . ...........................

NEW ZEALAND. Unlike true spinach in type and in that it thrives during hot weather and in any soil rich or poor. The tender shoots are of good quality and may be cut throughout the summer. The plant becomes very large and spreading. The leaves are comparatively small, broad and pointed. Plant three or four seeds in hills two feet apart each way. Germination of the seed can be hastened by soaking the seed in warm water for twenty-four hours..

oz. $1 / 5$ lb.

$.10 \quad .20$

SALSIFY, or VEGETABLE OYSTER.
SORREL (Large Leaf French). 10\% 


\section{TOMATO}

Sow in hotbeds in March. Transplant to open ground when the weather becomes warm and settled, or sow in the open ground in May and transplant when plants are six inches high. The richer the soil the more handsome and plentiful the fruit; but to have the fruit ripen as early as possible, select rather light soil and a sunny location. Plant in hills four feet apart each way. After the fruit is set, pinch off the ends to check growth; gradually strip off the leaves to expose the fruit to the sun.

Packets of each variety, 10 cents.

\section{EARLY RED VARIETIES}

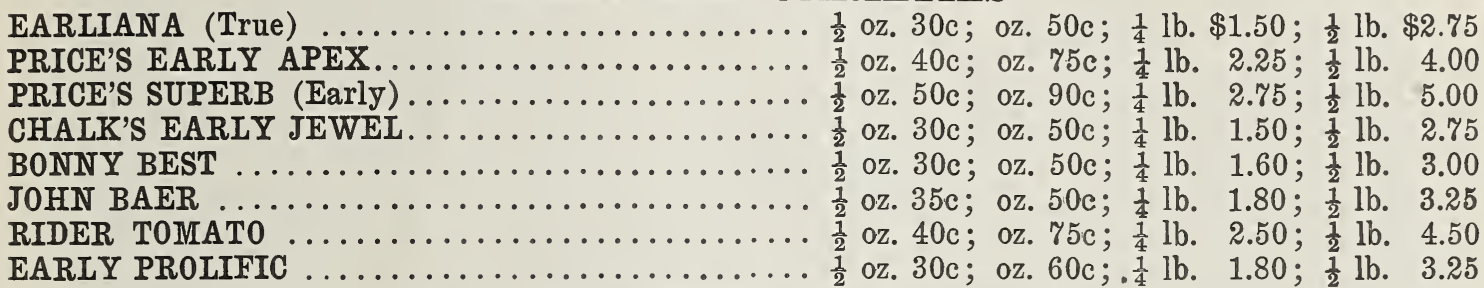

\section{GENERAL CROP - RED VARIETIES}

PRICE'S IIAIN CROP.................. $\frac{1}{2}$ oz. 25c; oz. $40 \mathrm{c} ; \frac{1}{4}$ lb. $\$ 1.35 ; \frac{1}{2}$ lb. $\$ 2.50$

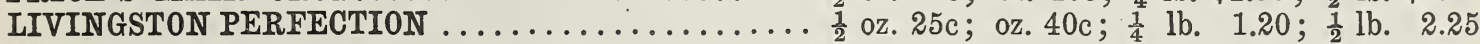
MATCHLESS. .................... $\frac{1}{2}$ oz. $30 \mathrm{c} ;$ oz. $50 \mathrm{c} ; \frac{1}{4} \mathrm{lb} .1 .50 ; \frac{1}{2} \mathrm{lb} .2 .75$ GREATER BALTIMORE ................ $\frac{1}{2}$ oz. $25 \mathrm{c} ;$ oz. $40 \mathrm{c} ; \frac{1}{4} \mathrm{lb} .1 .20 ; \frac{1}{2} \mathrm{lb} .2 .25$ SELECTED STONE ................... $\frac{1}{2}$ oz. $30 \mathrm{c} ;$ oz. $50 \mathrm{c} ; \frac{1}{4} \mathrm{lb} .1 .70 ; \frac{1}{2} \mathrm{lb} .3 .25$ DWARF STONE .................... $\frac{1}{2}$ oz. $25 \mathrm{c} ;$ oz. $40 \mathrm{c} ; \frac{1}{4} \mathrm{lb} .1 .40 ; \frac{1}{2} \mathrm{lb} .2 .50$ PONDEROSA. ........................ $\frac{1}{2}$ oz. $40 \mathrm{c} ;$ oz. $70 \mathrm{c} ; \frac{1}{4} \mathrm{lb} .2 .25 ; \frac{1}{2} \mathrm{lb} .4 .00$ RED HUSK OR GROUND CHERRY........... $\frac{1}{2}$ oz. $30 \mathrm{c}$; oz. $50 \mathrm{c} ; \frac{1}{4}$ lb. 1.70 RED PEAR (Shaped).................. $\frac{1}{2}$ oz. $25 \mathrm{c} ;$ oz. $40 \mathrm{c} ; \frac{1}{4} \mathrm{lb} .1 .50$

\section{PINK VARIETIES}

PRICE'S EARLY PINK. ................. $\frac{1}{2}$ oz. $30 \mathrm{c} ;$ oz. $50 \mathrm{c} ; \frac{1}{4} \mathrm{lb} . \$ 1.50 ; \frac{1}{2} \mathrm{lb} . \$ 2.75$

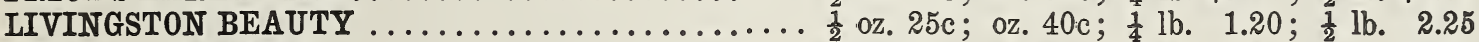
DWARF CHAMPION ................... $\frac{1}{2}$ oz. $30 \mathrm{c} ;$ oz. $50 \mathrm{c} ; \frac{1}{4} \mathrm{lb} .1 .50 ; \frac{1}{2} \mathrm{lb} .2 .75$

\section{GOLDEN OR YELLOW VARIETIES}

GOLDEN PONDEROSA ......................... $\frac{1}{2}$ 0z. 30c; oz. 50c; $\frac{1}{4} 1 \mathrm{lb} . \$ 1.70$ GOLDEN QUEEN ........................... $\frac{1}{2}$ oz. 30 c; oz. 50 c; $\frac{1}{4} l$ lb. $\$ 1.70$ YELLOW PLUM ............................. $\frac{1}{2}$ 0z. 30 c; oz. 50 c; $\frac{1}{4} 1 \mathrm{lb} . \$ 1.70$ YELLOW PEAR (Shaped) ........................ $\frac{1}{2}$ oz. $30 \mathrm{c} ;$ oz. $50 \mathrm{c} ; \frac{1}{4} 1 \mathrm{lb} . \$ 1.70$ YELLOW HUSK OR GROUND CHERRY................. $\frac{1}{2}$ oz. $25 \mathrm{c} ;$ oz. $40 \mathrm{c} ; \frac{1}{4} \mathrm{lb} .1 .50$

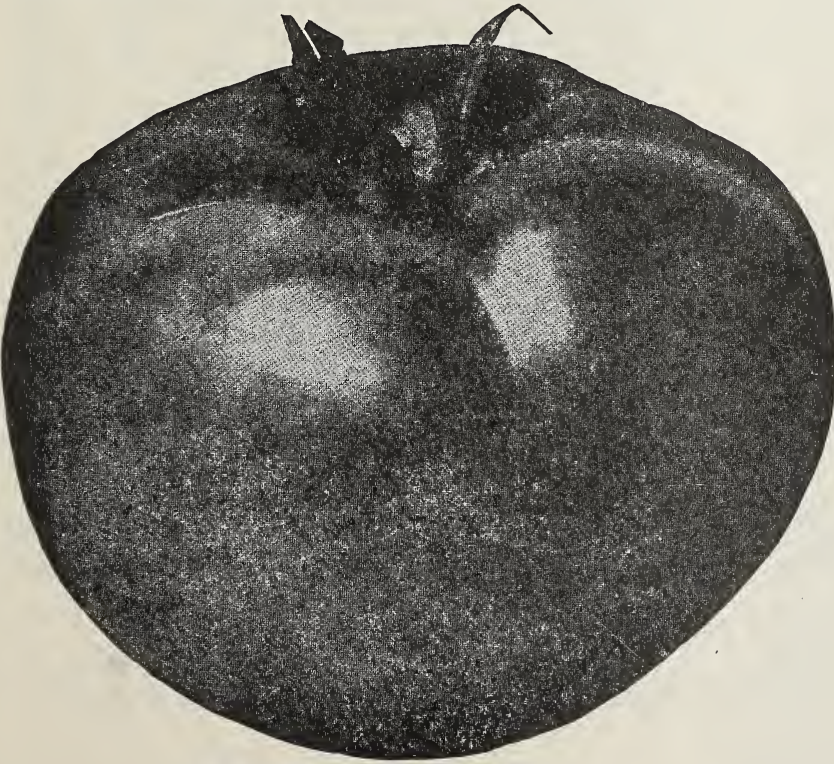

SELECTED STONE TOMATO

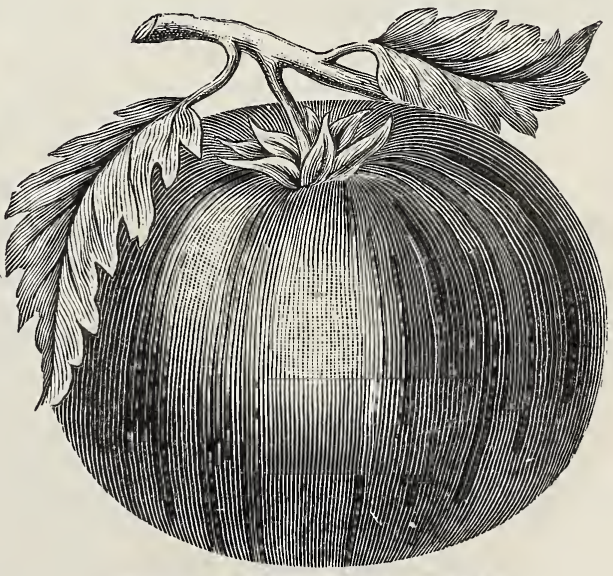

PRICE'S NEW PINR TOMATO

Tomato Plants by the Dozen, Hundred or Thousand Ready in May. See Page 22 


\section{TOBACCO}

CONNECTICUT SEED LEAF............................. per pkt. 10c; oz. 40c HAVANA. per pkt. 10c; oz. 50c

\section{TURNIP}

Packets of all varieties, 5c each.

PRICE'S EUREKA ......................... oz. $15 \mathrm{c} ; \frac{1}{4} \mathrm{lb} .40 \mathrm{c} ; \frac{1}{2} \mathrm{lb} . \$ 0.75 ; \mathrm{lb} . \$ 1.25$

BENDER. ............................. oz. $15 \mathrm{c} ; \frac{1}{4} \mathrm{lb} .40 \mathrm{c} ; \frac{1}{2} \mathrm{lb} . \quad .75 ; \mathrm{lb} .1 .25$

EARLY SNOWBALL ...................... Oz. $15 \mathrm{c} ; \frac{1}{4} \mathrm{lb} .30 \mathrm{c} ; \frac{1}{2} \mathrm{lb} . \quad .40 ; \mathrm{lb} . \quad 80$

EX. EA. WHITE MIILAN.................... oz. $20 \mathrm{c} ; \frac{1}{4} \mathrm{lb} .30 \mathrm{c} ; \frac{1}{2} \mathrm{lb} . \quad .45 ; \mathrm{lb} . \quad 90$

EX. EA. PURPLE TOP MIILAN.................. oz. $20 \mathrm{c} ; \frac{1}{4} \mathrm{lb} .30 \mathrm{c} ; \frac{1}{2} \mathrm{lb} . \quad .45 ; \mathrm{lb} . \quad .90$

IMPROVED WHITE EGG..................... oz. $15 \mathrm{c} ; \frac{1}{4} \mathrm{lb} .30 \mathrm{c} ; \frac{1}{2} \mathrm{lb} . \quad .40 ; \mathrm{lb} . \quad .80$

PURPLE TOP STRAP LEAF.................... oz. $15 \mathrm{c} ; \frac{1}{4} \mathrm{lb} .25 \mathrm{c} ; \frac{1}{2} \mathrm{lb} . \quad .35 ; \mathrm{lb} . \quad 60$

PURPLE TOP WHITE GLOBE................. Oz. $15 \mathrm{c} ; \frac{1}{4} \mathrm{lb} .30 \mathrm{c} ; \frac{1}{2} \mathrm{lb} . \quad .40 ; \mathrm{lb} . \quad .70$

LARGE WHITE GLOBE..................... oz. $15 \mathrm{c} ; \frac{1}{4} \mathrm{lb} .30 \mathrm{c} ; \frac{1}{2} \mathrm{lb} . \quad .40 ; \mathrm{lb} . \quad .70$

YELLOW GLOBE .......................... oz. $15 \mathrm{c} ; \frac{1}{4} \mathrm{lb} .30 \mathrm{c} ; \frac{1}{2} \mathrm{lb} . \quad .40 ; \mathrm{lb} . \quad .70$

AIIIBER GLOBE $\ldots \ldots \ldots \ldots \ldots \ldots \ldots \ldots \ldots \ldots \ldots$ oz. $15 \mathrm{c} ; \frac{1}{4} \mathrm{lb} .30 \mathrm{c} ; \frac{1}{2} \mathrm{lb} . \quad .40 ; \mathrm{lb} . \quad .70$

ROBSON'S GOLDEN BALL..................... oz. $15 \mathrm{c} ; \frac{1}{4} \mathrm{lb} .30 \mathrm{c} ; \frac{1}{2} \mathrm{lb} . \quad .40 ; \mathrm{lb} . \quad .70$

WHITE STONE $\ldots \ldots \ldots \ldots \ldots \ldots \ldots \ldots \ldots \ldots \ldots \ldots$ oz. $15 \mathrm{c} ; \frac{1}{4} \mathrm{lb} .30 \mathrm{c} ; \frac{1}{2} \mathrm{lb} . \quad .40 ; \mathrm{lb} . \quad .70$

YELLOW STONE .......................... oz. $15 \mathrm{c} ; \frac{1}{4} \mathrm{lb} .30 \mathrm{c} ; \frac{1}{2} \mathrm{lb} . \quad .40 ; \mathrm{lb} . \quad .70$

LONG WHITE COW HORN.................... Oz. $15 \mathrm{c} ; \frac{1}{4} \mathrm{lb} .30 \mathrm{c} ; \frac{1}{2} \mathrm{lb} . \quad .50 ; 1 \mathrm{lb} . \quad .90$

\section{RUTA BAGA OR SWEDE}

AMERICAN PURPLE TOP..................... oz. $15 \mathrm{c} ; \frac{1}{4} \mathrm{lb} .25 \mathrm{c} ; \frac{1}{2} \mathrm{lb} .40 \mathrm{c} ; \mathrm{lb} . \$ 0.90$ IMPROVED LONG ISLAND .......................... oz. $15 \mathrm{c} ; \frac{1}{4} \mathrm{lb} .25 \mathrm{c} ; \frac{1}{2} \mathrm{lb} .40 \mathrm{c} ; \mathrm{lb} .90$ SKIRVINGS. . . ........................... oz. $15 \mathrm{c} ; \frac{1}{4} l \mathrm{lb} .25 \mathrm{c} ; \frac{1}{2} \mathrm{lb} .40 \mathrm{c} ; \mathrm{lb} . \quad .80$

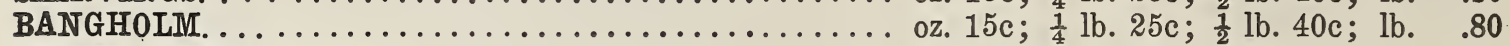
WHITE RUSSIA .......................... oz. $15 \mathrm{c} ; \frac{1}{4} \mathrm{lb} .25 \mathrm{c} ; \frac{1}{2} \mathrm{lb} .40 \mathrm{c} ; \mathrm{lb} . \quad 80$

\section{HERB SEEDS}

ANISE. ........................... per pkt. $10 \mathrm{c} ; \frac{1}{2}$ oz. $15 \mathrm{c} ;$ oz. $25 \mathrm{c} ; \frac{1}{4} \mathrm{lb} . \$ 0.60$ BORAGE. ............................. per pkt. $5 \mathrm{c} ; \frac{1}{2}$ oz. $10 \mathrm{c} ;$ oz. $20 \mathrm{c} ; \frac{1}{4} 1 \mathrm{lb} . \quad .50$ CARAWAY. . . ....................... per pkt. $5 \mathrm{c}$; $\frac{1}{2}$ oz. $10 \mathrm{c}$; oz. $15 \mathrm{c} ; \frac{1}{4} \mathrm{lb} . \quad .40$ CORIANDER. . . ....................... per pkt. $5 \mathrm{c} ; \frac{1}{2}$ oz. $10 \mathrm{c} ;$ oz. $15 \mathrm{c} ; \frac{1}{4} \mathrm{lb} . \quad .40$ DILI. .............................. per pkt. $5 \mathrm{c} ; \frac{1}{2}$ oz. $10 \mathrm{c} ; \mathrm{oz} .20 \mathrm{c} ; \frac{1}{4} \mathrm{lb} . \quad .50$ FENNEL (Sweet) ........................ per pkt. $5 \mathrm{c} ; \frac{1}{2}$ oz. 10c; oz. 20c; $\frac{1}{4}$ lb. .50 MARJORAMI (Sweet) ................... per pkt. $5 \mathrm{c} ; \frac{1}{2} \mathrm{oz} .20 \mathrm{c} ; 0 \mathrm{oz} .35 \mathrm{c} ; \frac{1}{4} \mathrm{lb} .1 .00$ ROSEMARY. .......................... per pkt. $10 \mathrm{c} ; \frac{1}{2}$ oz. $20 \mathrm{c} ;$ oz. $40 \mathrm{c} ; \frac{1}{4}$ lb. 1.50 SAVORX, SUMMER ....................... per pkt. $5 \mathrm{c} ; \frac{1}{2}$ oz. $10 \mathrm{c}$; oz. $20 \mathrm{c} ; \frac{1}{1 \mathrm{lb} .} \quad .60$ SAGE. ........................... per pkt. $5 \mathrm{c}$; $\frac{1}{2}$ oz. $20 \mathrm{c}$; oz. $35 \mathrm{c} ; \frac{3}{4} \mathrm{lb} .1 .25$

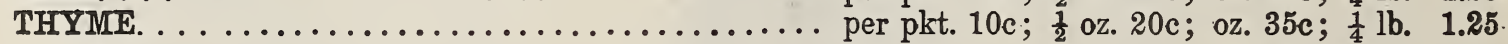

\section{A COMPLETE LINE OF BIRD SEED, REMEDIES AND SUPPLIES}

MIXED BIRD SEED (fresh and clean). Mixture contains Sicily Canary, Essex Rape, Millet and a very little Russian Hemp, just enough to keep your birds in good condition. Per lb.

CANARY AND RAPE, SICILY CANARY, ESSEX RAPE, RUSSIAN HEMP, SUNFLOWER, UNHULLED RICE, PARROT SEED MIXED, MIILIET, BIRD LETTUCE and WASHED SILVER. SAND. Per lb.

We will quote special price on the above in quantity.

CUTTLE BOIFE, BIRD MANNA, SHEPARD'S SOIG RESTORER, BIRD TONIC and LICE SALVE. 


\section{Annuals, Biennials and Perennials and Culture Directions.}

Annuals are plants which, raised from seed, perfect the flowers, ripen their seed and perish the same season. There are some, such as Verbena, Eschscholtzia, cultivated as annuals, that are only such in a northern climate, being in their own congenial region biennials and perennials. Biennials are those which grow the first season, flower the next, and then die. They may sometimes be induced to flower for two or three suc cessive seasons, by preventing them from going to seed. Perennials are those which live and continue to blossom several years. If planted very early in borders, or brought forward in windows or hot-beds, they will bloom the first season. Many of them are hardy enough to stand the coldest of our Winters, dying down every season, and coming up in the Spring from the old roots.

Annuals are divided in two classes, Hardy and Half-hardy. The Hardy can be sown in April or May in the open ground, or for early flowering started in pots or pans in the house, greenhouse or hot-bed, and planted out as soon as ground is warm. Half-hardy should be started in the house, greenhouse or hot-bed. and planted out in beds or borders in May, or they can be sown in the open groind abuut the middle of May.

Biennials and Perennials may be sown in the Spring as early as annuals - they need the same treatment, and should be planted out the following Autumn in situations where they are to flower. Can be sown in the Autumn, selecting as cool and damp a bed as possible, keeping the ground shaded and moist by frequent waterings until plants appear.

Greenhouse seeds should be sown in pots or pans half full of drainage; cover this with rough siftings of mould, and the surface with fine soil, moisten, using a fine rose. Sow seeds evenly over the surface, cover very lightly, keeping from the rays of the sun until the plants show the third leaf, then transplant into seed pans three-fourths of an inch apart, keeping them under a frame until perfectly recovered, giving a little air to strengthen them. As soon as they begin to touch each other transplant into small pots and keep in a cool, airy part of the greenhouse near the glass, repotting them when necessary.

Great care should be taken in the preparation of the soil for sowing the smaller kinds of seeds; the ground should be made rich with sheep manure, some good fertilizer or thoroughly rotted manure, dug deeply, well raked, made very fine and pliable, so the roots can penetrate and the soil will not bake in the sun after watering. The seed should be sown very thinly, covered very lightly and pressed firmly and carefully into the ground. As a rule small seeds should not be covered over one-eighth of an inch deep, the larger ones, like Morning Glory. Balsams, etc., three-fourths of an inch; very fine seeds should be sown on the surface, merely dusting them over with a little earth and gently press down with a smooth board. Keep the ground free from weeds and loosen the ground often, so rain will penetrate. In watering, use water that is not too cold; distribute it in the evening through a rose, so it will not wash the soil over the growing seed or injure the foliage or expanding buds.

Hot-bed for Seedlings. In making a hot-bed the preparation of manure is a matter of importance, and it should be well worked previous to being used, if the bed be expected to retain its usefulness for any length of time. If obtained fresh, and found to be too dry, it should be well watered and thrown lightly together to ferment; this will take place in a few days, and from three to four days afterward it should be completely turned, shaken and mixed; a second turning may be required, although one is usually sufficient. When thus cleaned of rankness the bed can be made. The place should be chosen where it is dry underneath, sheltered from the north as much as possible, and fully exposed to the sun. It should be built up from two feet six inches to four feet high, and wider by six inches every way than the frame to be placed upon it. The manure should be well shaken and mixed, while being put together, and firmly pressed by the feet. The frame should be placed on this and kept closed until the heat rises, and three or four inches of sifted sand or light soil placed on the surface of the bed. In a few days it will be ready for use; but air should be given night and day while there is any danger from the rank steam. If the sand or soil are drawn away from the sides of the bed, they should be replaced. When the hot-bed is used for seed only, nothing further is necessary; the heat of which will soon cause them to germinate; as this will after some time decline, a lining should be added, that is, fresh, hot, fermented, but not rank, manure, applied abour a foot in width all around the bed; this will renew its strength.

Cold Frames are formed by placing an ordinary hot-bed frame upon a bed of light, rich soil in some warm, sheltered place in the garden, where it will be protected from cold winds. They should both be shaded from the sun by mats during the middle of the day and covered at night.

\section{Vegetable Plants, Flower Plants and Roots}

Cabbage and Cauliflower Plants, by the dozen, 100, 1,000 or in larger quantities.

Brussel Sprout Plants, by the dozen or 100 .

Celery Plants, by the $100,1,000$ or in larger quantitice.

Egg Plants, grown in pots, by the dozen or hundred.

Pepper Plants, Large Bell, Ruby King, and Crimson Giant.

Tomato Plants, Earliana, Chalk's Jewel, Livingston's Beauty, Dwarl Stone, Dwart Champion, Selected Stone, by the dozen, 100 , 1,000 and in larger quantities.
Sweet Potato Plants, ready in June, by the 100 .

Aster Plants, in separate and mixed colors, by the dozen or 100. Salvia Plants, by the dozen.

Asparagus Roots, see page 4.

Rhubarb Roots, may be planted in the Spring or Fall. Set three feet apart each way.

Horse Radish Sets, by the dozen or 100.

Price of the above on application.

\section{Use Pulverized Sheep Manure For Your Garden}




\title{
FLOWER SEEDS
}

\section{Choice and Standard Varieties}

\author{
POST PAID, ON FLOWER SEEDS IN THE UNITED STATES.
}

a. Annual. ha. Hardy Annual. b. Biennial. p. Perennial. hhp. Half Hardy Perennial. gh. Green House.

a ACROCLINIUM (Everlasting Flower)

Single Mixed .................... \$0.05 $\$ 0.20 \$ 0.80$

Double Mixed ................... $\quad .10 \quad .30 \quad .90$

a AGERATUM (Floss Flower)

Mixed ............................. $\quad .05 \quad .20 \quad .80$

Blue Porfection .................. . . $10 \quad .40 \quad .80$

Imperial Dwarf Blue.............. .05 $.25 \quad 1.00$

a ALYSSUM

Sweet (Tall) ................... . .05 $\quad .15 \quad .40$

Little Gem (Carpet of Snows)......

Tom Thumb (Erect).............. . .05 $\quad .20 \quad .60$

a AMARANTHUS

Tricolor (Joseph's Coat) $\ldots \ldots \ldots \ldots$. $\quad .05 \quad .20 \quad .60$

Caudatus (Love Lies Bleeding)....

$\begin{array}{lllll}\text { Cruentus (Prince's Feather)....... } & .05 & .20 & .60\end{array}$

p ANTIRRHINUM (Snapdragon)

$\begin{array}{lllll}\text { Tall Mixed } \ldots \ldots \ldots \ldots \ldots \ldots \ldots \ldots \ldots & .05 & .30 & .80 \\ \text { Dwarf Mixed } \ldots \ldots \ldots \ldots \ldots \ldots \ldots \ldots & .05 & .30 & .80\end{array}$

Semi-Dwarf. . . . . . . . . . . . . . . . .

p AQUILEgIA (Columbine)

Single Mixed ................... . .05 $\quad .25 \quad .60$

Double Mixed $\ldots \ldots \ldots \ldots \ldots \ldots \ldots \ldots \ldots, \quad .05 \quad .30 \quad .70$

a ASTER

Semple's Branching Mixed........ $\quad .10 \quad .40 \quad 1.50$

Giant Crego Mixed................. $.10 \quad .60 \quad 2.00$

Price's Large Branching Mixed.... $\quad .10 \quad .50 \quad 1.50$

Royal Mixed ......................... $10 \quad .60 \quad 2.00$

Queen of Market Mixed........... $.10 \quad .50 \quad 1.50$

Separate Colors of the above pkt., 10c.

BALLOON VINE (Love in a Buff)

(Cardiospermun) . . ............. .05

a BALSAM (Ladies' Slipper)

Choice Double Mixed...............

Striped and Spotted................

Double White ....................

Spotted. . . ......................

BELLIS (Perennis) (Double Daisy)

Mixed. . . ........................

Pink. . . ........................

White. . . ......................

a CACALIA (Tassel Flower)

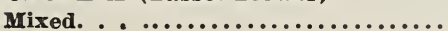

Scarlet. . . . . . . . . . . . . . . . . . . .

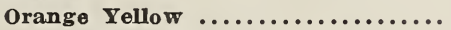

CALENDUla (Marigold) Pot

Orange Giant ....................

Mixed.

Prince of Orange.

CALLEOPSIS

Bicolor. ....

Finest Mixed

p

$\begin{array}{lll}.05 & .20 & .50\end{array}$

$\begin{array}{lll}.05 & .20 & .60\end{array}$

$\begin{array}{lll}.10 & .30 & 1.00\end{array}$

$\begin{array}{lll}.10 & .20 & .60\end{array}$

$\begin{array}{lll}.10 & 1.00 & 4.00\end{array}$

$\begin{array}{lll}.10 & 1.00 & 4.00\end{array}$

$\begin{array}{lll}.10 & 1.00 & 4.00\end{array}$

$\begin{array}{lll}.10 & .30 & 1.00\end{array}$

$\begin{array}{lll}.10 & .30 & 1.00\end{array}$

$\begin{array}{lll}.10 & .30 & 1.00\end{array}$

$\begin{array}{lll}.05 & .15 & .40\end{array}$

$\begin{array}{lll}.05 & .15 & .40\end{array}$

$\begin{array}{lll}.05 & .15 \quad .40\end{array}$ p CANTERBURY BELLS

Single Mixed .................... . .05 $\quad .25 \quad .80$

Double Mixed ................... . . $10 \quad .40 \quad 1.40$

Cup and Saucer Mixed............ . . $10 \quad .40 \quad 1.40$

CANDYTUFT

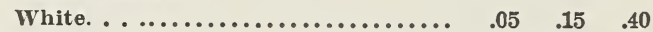

Rose. ........................ $.05 \quad .15 \quad .40$

Lavender. . . ............... . .05 $\quad .15 \quad .40$

Mixed........................ .0 .0 $\quad .15 \quad .40$

a CANARY BIRD VINE............. .0 $05 \quad .15 \quad .40$

CANNA

Mixed Sorts .................. $\quad .10 \quad .15 \quad .30$

p CARNATION

Marguerite.................. $\quad .10 \quad .40 \quad 1.50$

Hardy Border Double Mixed....... $\quad .10 \quad .40 \quad 1.50$

Vienna Double Mixed............. $\quad . \quad 10 \quad .40 \quad 1.50$

a CELOSIS (Cockscomb)

Dwarf Mixed .................. $\quad .10 \quad .40 \quad 1.50$

Tall Mixed ..................... $10 \quad .40 \quad 1.50$

a CENTAUREA (Bachelor's Button) (Cornflower)

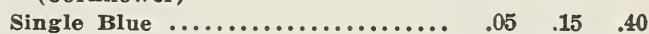

Double Blue .................. .05 $\quad .30 \quad 1.00$

Mixed. . . . . . . . . . . . . . . . . . . $\quad .40$

a CLARKIA

Double Mixed ................. . .05 $\quad .25 \quad .80$

Single Mixed $\ldots \ldots \ldots \ldots \ldots \ldots \ldots \ldots \ldots, \quad .05 \quad .25 \quad .80$

a COBAEA

Scandens (Purple) .............. $.05 \quad .25 \quad .80$

Mixed (White and Blue) .......... $.05 \quad .25 \quad .80$

a COLEUS

Mixed Hybrids ............... .25

Cosmos

Early Flowering Mixed........... .05

Pink Lady Lenox............... . .05

a CYPRESS VINE

Scarlet $\ldots \ldots \ldots \ldots \ldots \ldots \ldots \ldots \ldots \ldots, .05$

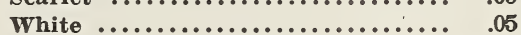

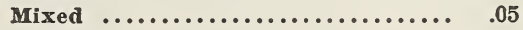

a DAHLIA

Fine Single Mixed............... . . $10 \quad .35 \quad 1.40$

Fine Double Mixed............... $\quad .10 \quad .351 .40$

DELPHINIUM (Larkspur)

Formosum (Blue) .................. . $10 \quad .602 .00$

Album (White) ............... . $10 \quad .20 \quad .60$

Single Mixed ................... $\quad .10 \quad .30 \quad .80$

Dwarf Rocket Mixed............ . . $05 \quad .15 \quad .40$

DIANTHUS (Pinks)

$\begin{array}{lll}.05 & .20 & .60\end{array}$

$.05 \quad .15 \quad .50$

Laciniatus Single $\ldots \ldots \ldots \ldots \ldots \ldots, .05$

Heddewegii Single ............... .05

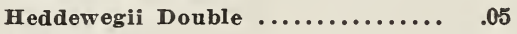

Double Mixed ................... . 10

$\begin{array}{llll}.05 & .15 & .50 & \text { p }\end{array}$

Single Mixed $\ldots \ldots \ldots \ldots \ldots \ldots \ldots \ldots \ldots . . .10$

$.30 \quad .80$ 
D Digitalis (Foxglove)

Mixed Colors .................... . $10 \quad .30 \quad 1.00$

Mixed Spotted ................... . .10 .30 1.00

Purple...$\ldots \ldots \ldots \ldots \ldots \ldots \ldots \ldots \ldots$. . .10 $\quad .30 \quad 1.00$

Rose .......................... . .10 .30 1.00

White ....................... . .10 $.30 \quad 1.00$

Mammoth Mixed ................. .10 . $35 \quad 1.25$

a DOLICHOS (Hyacinth Bean) (Climber)

White ........................ .05

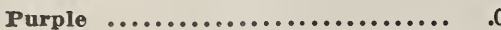

ESCHSCHOLTZIA (California Poppy)

Mixed Colors ....................

Californica (Yellow) $\ldots . . \ldots \ldots \ldots \ldots$

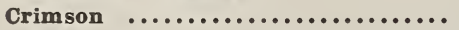

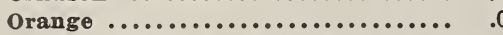

a EUPHORBIA (Snow on Mountain).

ap FORGET-ME-NOT (MYOsotis)

Royal Blue ................... .10 .40 1.50

Blue (Everblooming) ............ . $10 \quad .40 \quad 1.50$

Mixed ......................... .05 $\quad .30 \quad 1.00$

a FOUR O'CLOCK (Marvel of Peru)

Mixed Colors .....................

GAILLARDIA (Blanket Flower)

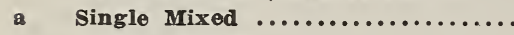

a Double Mixed ...................

Grandiflora Mixed ................ GODETIA

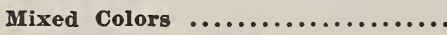

Dwarf Mixed Colors...............

a GOURD (Ornamental)

Mixed Sorts ...................... . .05 $\quad .10 \quad .30$

Bottle $\ldots \ldots \ldots \ldots \ldots \ldots \ldots \ldots \ldots \ldots \ldots \ldots, \quad .05 \quad .10 \quad .30$

Dipper ...................... .05 $\quad .10 \quad .30$

Hercules Club .................. $.05 \quad .10 \quad .30$

African Pipe ................... . .0 $\quad .10 \quad .30$

GYPSOPHILA (Baby's Breath)

a Grandifiora Elegans $\ldots \ldots \ldots \ldots \ldots \ldots \ldots, .05 \quad .20 \quad .60$

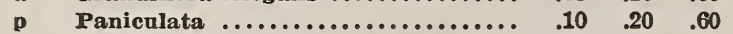

a HELICHRYSUM (Straw Flower)

Double Mixed .................. . .0 $\quad .15 \quad .40$

gh HELIOTROPE

Mixed Colors

Regal (Dwarf) $\ldots \ldots \ldots \ldots \ldots \ldots \ldots$

HOLLYHOCK

Single Mixed $. . . \ldots \ldots \ldots . . . \ldots \ldots$.

Double Mixed ...................

Fringed Mixed ...................

Donble White ....................

Double Rose .....................

Double Red .....................

Double Yellow ...................

IVY (Kenilworth) ..................

ICE PLANT (Mesembryanthemum)

JAPANESE HOP (Humulus)

Variegated Foliage ..............

a KOCHIA (Mexican Fire Bush).....

hhp LANTANA

Mixed Colors

ha LINUM (Scarlet Flax)

Grandiflorum Rubrum

p LOBELIA

Crystal Palace Compacta..........

LUPINUS

Mixed Colors

Deep Blue $\ldots . . . . . . . . . . . . . .$.

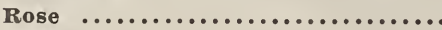

White .........................

p LYCHNIS (Rose Campion, Jerusalem Cross)

Chalcedonica Scarlet .............

Mixed Colors a MARIGOLD

African Lemon Queen............ . .05 $\quad .30 \quad .80$

African Double Mixed............. $\quad .05 \quad .30 \quad .80$

$\begin{array}{lllll}\text { French Dwart Double Mixed...... } & .05 & .30 & .80\end{array}$

a MIGNONETTE

Sweet $\ldots \ldots \ldots \ldots \ldots \ldots \ldots \ldots \ldots \ldots \ldots, \quad .05 \quad .10 \quad .30$

Large Flowered ................ . .10 $\quad .30 \quad .80$

a MImosA (Sensitive Plant)....... . . . $05 \quad .20 \quad .50$

hhp MIMULUS

Moschatus (Musk Plant)............

Tigrinus (Monkey Flower)..........

a MOMORDICA

Balsam Apple

Balsam Pear

MOON FLOWER (Ipomea)

Grandiflora $. . . \ldots \ldots \ldots \ldots \ldots \ldots . . .$.

Cardinal Climber ................

Imperial Japanese ................

MORNING GLORY (Convolvulus Major)

Mixed Colors .................. .05

NICOTIANA

Affinis (White) $\ldots . \ldots \ldots \ldots \ldots \ldots$.

Sanderae (Mixed) .................

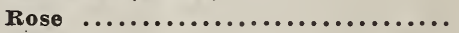

ha NIGELLA (Love-in-a-Mist) (Devilin-Bush)

$\begin{array}{lll}.05 . & .20 & .50\end{array}$

$\begin{array}{lll}.05 & .15 & .40\end{array}$

$\begin{array}{lll}.05 & .20 & .00\end{array}$
Double Mixed

Blue

\section{a NASTURTIUM}

Tall Mixed

$1 / 4$ lb. $40 \mathrm{c} ; 1 \mathrm{~b} . \$ 1.25$

Dwarf Mixed ...........

$1 / 4$ lb. $50 \mathrm{c} ; 1 \mathrm{~b} . \$ 1.50$

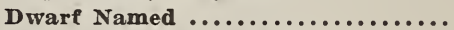

Aurora (Terra Cotta).............

Beauty (Yellow and Scarlet)........

Bronzecolored....................

Spotted ........................

Coeruleo Roseum (Blush Red).......

Crystal Palace Gem (Blotched).....

Dark Furple (Spencer)............

David Burpee (Spencer) (Red).....

$\begin{array}{lll}.10 & .70 & 2.50\end{array}$

$\begin{array}{lll}.10 & .40 & 1.50\end{array}$

$\begin{array}{lll}.10 & .40 & 1.50\end{array}$

$\begin{array}{lll}.10 & .40 & 1.50\end{array}$

$\begin{array}{lll}.10 & .40 & 1.50\end{array}$

$\begin{array}{lll}.10 & .40 & 1.50\end{array}$

$\begin{array}{lll}.10 & .40 & 1.50\end{array}$

$\begin{array}{rrr}.10 & .40 & 1.50\end{array}$

$\begin{array}{lll}.10 & .75 & 3.00\end{array}$

$.05 \quad .20 \quad .60$

$\begin{array}{lll}.05 & .20 & .60\end{array}$

$\begin{array}{lll}.05 & .15 & .40\end{array}$

.05

.05

.20.

.05

$.15 \quad .40$

.10

$.40 \quad 1.50$

.05

.10

.10

.15

.20

.50

$.20 \quad 50$

p PANSY

Price's Giant Large Flowering

Mixed (35 Varieties).............

Price's Superb Mixed (25 Varieties)

Price's Special Mixed (20 Varieties)

a PETUNIA

Single Mixed (Large Flowering)...

Bedding Sorts,

Rosy Morn .........

Snowball (White)

Brilliant .............

PHLOX

Drummondii Choice Mixed..........

Cuspidata (Star Phlox Mixed)......

Fimbriata (Fringed Mixed).........

Decussata (Mixed) .................

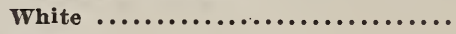

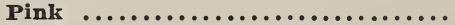

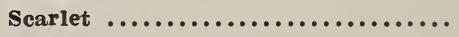

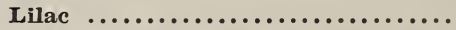

PHYSALIS (Chinese Lantern Plant)

POLEMONIUM (Jacob's Ladder)

Blue $\ldots \ldots \ldots \ldots \ldots \ldots \ldots \ldots \ldots \ldots . . .10$

POPPY

a Single Mixed

Double Mixed ...................

Carnation Flowered Mixed.........

Shirley Mixed

$\begin{array}{lll}.05 & .15 & .40\end{array}$

$\begin{array}{lll}.05 & .20 & .50\end{array}$

.05

.. .15

.05

. .15

.05

.05
.05

.05

.05

.05

.05

.10

.10

$1.25 \quad 5.00$

$1.00 \quad 4.00$

$\begin{array}{ll}.70 & 2.50\end{array}$

.05

.10

$.40 \quad 1.50$

$.80 \quad 3.00$

$\begin{array}{ll}.80 & 3.00\end{array}$

$\begin{array}{ll}.80 & 3.00\end{array}$

$.40 \quad 1.50$

$\begin{array}{lll}.05 & .30 & .80\end{array}$

$\begin{array}{lll}.05 & .40 & 1.00\end{array}$

$\begin{array}{lll}.10 & .40 & 1.00\end{array}$

$\begin{array}{lll}.10 & .80 & 3.00\end{array}$

$\begin{array}{lll}.10 & .40 & 1.50\end{array}$

$\begin{array}{lll}.10 & .40 & 1.50\end{array}$

$\begin{array}{lll}.10 & .40 & 1.50\end{array}$

$\begin{array}{lll}.10 & .40 & 1.50\end{array}$

$.10 \quad .40 \quad 1.00$

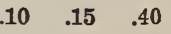

$\begin{array}{lll}.05 & .20 & .60\end{array}$

$\begin{array}{lll}.05 & .20 & .50\end{array}$

$\begin{array}{lll}.05 & .20 & .50\end{array}$

$\begin{array}{lll}.10 & .70 & 2.50\end{array}$

.05

$.20 \quad .50$ 


\begin{tabular}{|c|c|c|c|c|c|c|c|c|c|}
\hline & & & $1 / 40 z$ oz. & oz. & & & Pkt & 02. & oz. \\
\hline 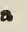 & Tulip (Scarlet) $\ldots \ldots \ldots \ldots \ldots \ldots \ldots$ & .05 & .30 & 1.00 & & Asta Ohn, & & & \\
\hline & Iceland Mixed...$\ldots \ldots \ldots \ldots \ldots$ & .10 & .40 & 1.50 & & Lavender Tinted Mauve........... & .10 & -. & .30 \\
\hline p & Oriental.... $\ldots \ldots \ldots \ldots \ldots \ldots \ldots$ & .10 & .40 & 1.50 & & Auroa, & & & \\
\hline a & $\begin{array}{l}\text { PORTULACA } \\
\text { Single Mixed } \ldots \ldots \ldots \ldots \ldots \ldots \ldots \ldots\end{array}$ & .05 & .25 & .75 & & $\begin{array}{l}\text { Orange Rose Striped on White.... } \\
\text { Blanche Ferry, }\end{array}$ & .10 & $\bullet$ & 30 \\
\hline & 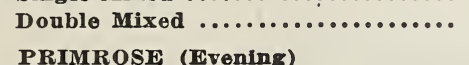 & .10 & .60 & 2.00 & & $\begin{array}{l}\text { Rose Pink, White Wings.......... } \\
\text { Countess Spencer, }\end{array}$ & .10 & .. & .30 \\
\hline & $\begin{array}{l}\text { Large White } \cdots \cdots \cdots \cdots \cdots \cdots \cdots \cdots \cdots \\
\text { PYRETHRUM }\end{array}$ & & .75 & $\cdots$ & & $\begin{array}{l}\text { White, Pink Edge............... } \\
\text { Dobbie's Cream, }\end{array}$ & .10 & .. & .30 \\
\hline 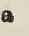 & Golden Feather .................. & .05 & .25 & .75 & & Creamy Yellow .................. & .10 & .. & .30 \\
\hline p & 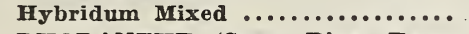 & .10 & .60 & 2.00 & & Helen Lewis, & & & \\
\hline a & $\begin{array}{l}\text { RHODANTHE (Swan River Ever- } \\
\text { lasting) }\end{array}$ & & & & & $\begin{array}{l}\text { Crimson Orange } \ldots \ldots \ldots \ldots \ldots \ldots \ldots \\
\text { Helen Pierce, }\end{array}$ & .10 & .. & .30 \\
\hline & $\begin{array}{l}\text { Mixed } \ldots \ldots \ldots \ldots \ldots \ldots \ldots \ldots \ldots \ldots \\
\text { RrCrNUs (Castor Oil Bean) } \ldots \ldots \ldots \ldots\end{array}$ & $\begin{array}{l}.10 \\
.05\end{array}$ & .30 & $\ddot{30}$ & & $\begin{array}{l}\text { White Veined, Mottled with Blue. } \\
\text { Illuminator, }\end{array}$ & .10 & $\cdots$ & .30 \\
\hline & $\begin{array}{l}\text { Cambodgiensis (almost Black), } 6 \mathrm{ft} \text {. } \\
\text { Philippinensis (Purplish Foliage).. }\end{array}$ & .05 & $\ddot{n}$ & $\begin{array}{l}.30 \\
.30\end{array}$ & & $\begin{array}{l}\text { Bright Glowing Salmon.......... } \\
\text { King Edward Spencer, }\end{array}$ & .10 & .. & .30 \\
\hline & $\begin{array}{l}\text { Sanguineus (Red Stem), } 8 \mathrm{ft} . \ldots \ldots \ldots \\
\text { Zanzibariensis (Bronze), } 12 \mathrm{ft} . \ldots \ldots\end{array}$ & .05 & -. & .30 & & $\begin{array}{l}\text { Crimson Scarlet } \ldots \ldots \ldots \ldots \ldots \ldots \\
\text { King Manuel, }\end{array}$ & .10 & .. & .30 \\
\hline & $\begin{array}{l}\text { Zanzibariensis }(\text { Bronze), } 12 \text { ft...... } \\
\text { Mixed (all sorts) } \ldots \ldots \ldots \ldots \ldots \ldots \ldots\end{array}$ & .05 & $\ddot{n}$ & $\begin{array}{l}.30 \\
.30\end{array}$ & & $\begin{array}{l}\text { King Manuel, } \\
\text { Maroon or Dark Chocolate........ } \\
\text { King White }\end{array}$ & .10 & $\cdots$ & .30 \\
\hline & $\begin{array}{l}\text { SALVIA (Scarlet } \text { Sage) } \\
\text { Splendens (Tall) } \ldots \ldots \ldots \ldots \ldots \ldots \ldots \ldots \ldots \\
\text { Bonfire (Dwarf) } \ldots \ldots \ldots \ldots \ldots \ldots \ldots\end{array}$ & $\begin{array}{l}.10 \\
.10\end{array}$ & $\begin{array}{l}.60 \\
.70\end{array}$ & $\begin{array}{l}2.00 \\
2.50\end{array}$ & & $\begin{array}{r}\text { Creamy } \\
\text { Primrose, }\end{array}$ & .10 & -. & .30 \\
\hline & Zurich (Dware Early) ............ & .15 & 1.25 & $\begin{array}{l}2.50 \\
5.00\end{array}$ & & $\begin{array}{l}\text { Cream and Ivory } \ldots \ldots \ldots \ldots \ldots \ldots \ldots \\
\text { Royal Purple, }\end{array}$ & .10 & .. & .30 \\
\hline & $\begin{array}{l}\text { SCABIOSA (Mourning Bride) } \\
\text { Mixed Colors } \ldots \ldots \ldots \ldots \ldots \ldots \ldots \ldots\end{array}$ & .05 & .20 & .60 & & $\begin{aligned} & \text { Deep Purple } \text { P................... } \\
& \text { Scarlet Emperor, }\end{aligned}$ & .10 & .. & .30 \\
\hline & $\begin{array}{l}\text { SCHIZANTHUS (Fringe Flower) } \\
\text { Mixed Colors } \ldots \ldots \ldots \ldots \ldots \ldots \ldots \ldots \ldots\end{array}$ & .05 & .20 & .60 & & $\begin{array}{l}\text { Deep Scarlet } \ldots \ldots \ldots \ldots \ldots \ldots \ldots \ldots \\
\text { Warrior, }\end{array}$ & .10 & $\cdots$ & .30 \\
\hline & $\begin{array}{l}\text { SHASTA DAISY } \\
\text { Mixed Colors } \ldots \ldots \ldots \ldots \ldots \ldots \ldots \ldots\end{array}$ & & & & & $\begin{array}{l}\text { Fine Maroon } \ldots \\
\text { Wedgwood, }\end{array}$ & .10 & $\cdots$ & .30 \\
\hline & $\begin{array}{l}\text { STOCKs (Ten Weeks) } \\
\text { Finest Mixed } \ldots \ldots \ldots \ldots \ldots \ldots \ldots \ldots\end{array}$ & .05 & .40 & 1.50 & & $\begin{array}{l}\text { Silvery Blue } . . . \ldots \ldots \\
\text { White, } \\
\text { Large White ........... }\end{array}$ & .10 & -. & .30 \\
\hline & $\begin{array}{l}\text { Cut and Come Again Mixed....... } \\
\text { sUNFLOWER (Helianthus) }\end{array}$ & $\begin{array}{l}.05 \\
.05\end{array}$ & $\begin{array}{l}.60 \\
.80\end{array}$ & $\begin{array}{l}2.00 \\
3.00\end{array}$ & $\mathbf{p}$ & $\begin{array}{l}\text { Large White } \cdots \ldots \ldots \\
\text { HARDY SWEET PEAS (Lat }\end{array}$ & .1 & -. & .30 \\
\hline & 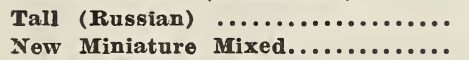 & $\begin{array}{l}.05 \\
.10\end{array}$ & & .10 & $\mathbf{a}$ & HUNBERGIA & .10 & .20 & .60 \\
\hline & Chrysanthemum Flowered (Double) & .10 & .25 & .80 & a & $\begin{array}{l}\text { Mixed Colors } \ldots \ldots \ldots \ldots \ldots \ldots \ldots \ldots \\
\text { VERBENA }\end{array}$ & .10 & .60 & .• \\
\hline & Double Yellow $\ldots \ldots \ldots \ldots \ldots \ldots \ldots$ & .10 & .25 & .80 & & 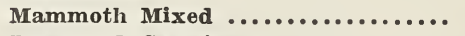 & .05 & .30 & 1.00 \\
\hline p & $\begin{array}{l}\text { SWEET WILLIAM } \\
\text { Single Mixed ......... }\end{array}$ & .10 & .20 & .. & & 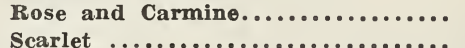 & .05 & .60 & 2.00 \\
\hline & 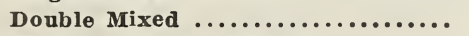 & .10 & .30 & .. & & 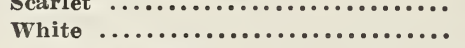 & $\begin{array}{l}.05 \\
.05\end{array}$ & $\begin{array}{l}.60 \\
.60\end{array}$ & $\begin{array}{l}2.00 \\
2.00\end{array}$ \\
\hline & Holborn Glory (Single) ............ & 10 & .25 & .. & a & WALLFLOWER & & & \\
\hline & $\begin{array}{l}\text { Newport Pink } \ldots \ldots \ldots \ldots \ldots \ldots \ldots \ldots \\
\text { Scarlet Beauty } \ldots \ldots \ldots \ldots \ldots \ldots \ldots \ldots\end{array}$ & .15 & •. & . & & … & .05 & .20 & $\cdots$ \\
\hline & 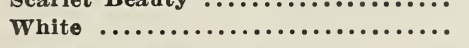 & $\begin{array}{l}.15 \\
.10\end{array}$ & $\ddot{30}$ & $\ddot{0}$ & & 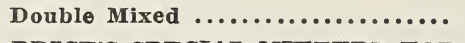 & .10 & . & .. \\
\hline & $\begin{array}{l}\text { SWEET PEAS } \\
\text { Price's Superb Mixed ( } 65 \text { Varieties) } \\
1 / 4 \text { lb. } 50 \mathrm{c} ; 1 / 2 \text { lb. } 80 \mathrm{c} ; 1 \mathrm{~b} . \$ 1.50\end{array}$ & .05 & .00 & .20 & a & $\begin{array}{l}\text { PRICE'S SPECIAL MIXTURE FOR } \\
\text { WILD FLOWER GARDEN..... } \\
\text { XERANTHEMUM }\end{array}$ & .05 & .15 & .30 \\
\hline & $\begin{array}{l}\text { Choice Mixed Spencer..................... } \\
1 / 4 \text { lb. } 90 \mathrm{c} ; 1 / 2 \text { lb. } \$ 1.60 ; \text { lb. } \$ 3.00\end{array}$ & .10 & .. & .30 & a & $\begin{array}{l}\text { Double Mixed } . . . \ldots \ldots \ldots \ldots \ldots \ldots \\
\text { ZINNIA (Youth and old Age) }\end{array}$ & .10 & .25 & .. \\
\hline & red or Spencer (Named & & & & & Flowered Mixture.... & .1 & .40 & 1.25 \\
\hline & & & & & & $\begin{array}{l}\text { ite (Giant) } \ldots \ldots \ldots \ldots \ldots \ldots \ldots \ldots \ldots \ldots \ldots \\
\text { mson (Giant) } \ldots \ldots \ldots \ldots \ldots \ldots \ldots \ldots\end{array}$ & $\begin{array}{l}.10 \\
.16\end{array}$ & $\begin{array}{l}.50 \\
.50\end{array}$ & $\begin{array}{l}1.50 \\
1.50\end{array}$ \\
\hline & $\begin{array}{l}\text { Apple Blossom, } \\
\text { Rose, Carmine Tinted............ }\end{array}$ & .10 & .. & .30 & & 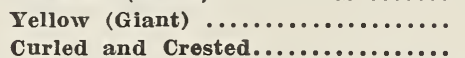 & .10 & .50 & $\begin{array}{l}1.80 \\
1.20\end{array}$ \\
\hline
\end{tabular}

POT LABELS, GARDEN LABELS, GARDEN REELS, AND GARDEN LINE.

ROUND PAINTED PLANT STICKS in lengths from one and one-half feet to six feet.

WOODEN PLANT TUBS, made of cedar, painted green, with iron hoops and drop handles.

12 in. high $\times 13 \frac{1}{2}$ dia............. $\$ 1.50$

17 in. high $x 18$ dia.............. $\$ 3.00$

14 in. high $\times 14 \frac{1}{2}$ dia............ 2.25

19 in. high $x 22$ dia............ 3.00

16 in. high $\times 16$ dia............. 2.50

20 in. high $\times 24$ dia................

3.50

We carry many varieties of Flower, Field and Garden Seeds, Agricultural Implements, Horticultural Implements, Garden Tools and Requisites, Fertilizers, Poultry Appliances, Dairy Supplies and Bird Supplies which we do not have room to list in this catalogue, on which we will be pleased to quote price on application. 


\section{SUMMER FLOWERING BULBS}

\section{DWARF CANNAS, NAMED VARIETIES.}

Each. Doz.

Each. Doz.

AUSTRIA (5 feet) (Green)

MIME. CROZY (3 feet) (Green)

$\$ 0.10-\$ 1.00$

Lemon Yellow, spotted Red... $\$ 0.10 \quad \$ 1.00$ CHARLES HENDERSON

Scarlet Orange, Yellow Border. $\$ 0.10 \quad \$ 1.00$

(4 feet) (Green)

Deep Crimson ............. $\quad .10 \quad 1.00$

DAVID HARUIM

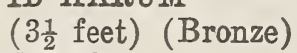

Red Dark ............. $\quad .10 \quad 1.00$

KING HUIMBERT

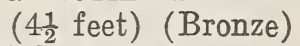

Bright Scarlet ............ $\quad .10 \quad 1.00$

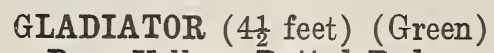

Deep Yellow, Dotted Red.... . $\quad .10 \quad 1.00$ PENNSYLVANIA

( 5 feet) (Green)

Vermillion Scarlet ........ $.10 \quad 1.00$

FLORENCE VAUGHAN

(5 feet) (Green)

Yellow, spotted Red......... $\quad .10 \quad 1.00$

\section{GLADIOLUS.}

Each. Doz. 100

CHOICE MIXED (Fine) ... $\$ 0.05 \$ 0.50 \$ 2.50$

NAIIED MIXTURES ..... . . $\quad .07 \quad .65 \quad 4.00$

SUPERB MIXED (Large) . . $\quad .10 \quad 1.00 \quad 6.00$

AIIERICA

Light Pink ......... . . .07 $\quad .65 \quad 4.00$

IVRS. FRANCIS KING

Brilliant Pink, Blazed

Vermillion ..........

$\begin{array}{lll}.06 & .60 & 3.50\end{array}$

\begin{abstract}
PEACE
Each. Doz. 100

White, Streaked Violet. $\$ 0.08 \$ 0.75 \$ 4.50$ YELLOW HAIMIIER

Pure Yellow .......... .08 $\quad .75 \quad 6.00$

EUROPE

White $\ldots \ldots \ldots \ldots \ldots . . .6 \quad .15 \quad 1.60$
\end{abstract}

\section{DAHIIAS. \\ We Have a Large Variety to Select From.}

DAHLIAS, Solid Colors, Large Double in Pink, White, Red, Yellow, Scarlet and Maroon. 20c each; $\$ 2.00$ per dozen.

SHOW OR FANCY. From $25 \mathrm{c}$ to $40 \mathrm{c}$ each; from $\$ 2.50$ to $\$ 3.00$ per dozen.

CACTUS DAHLIAS. From $25 \mathrm{c}$ to $35 \mathrm{c}$ each; $\$ 3.00$ per dozen.

NAIIED VARIETIES. From $35 \mathrm{c}$ to $50 \mathrm{c}$ each; $\$ 4.00$ per dozen.

\section{CALADIUM (Esculentum) (Elephant's Ear).}

Large Bulbs, 11 to 12 inches circumference. $40 \mathrm{c}$ each; $\$ 4.00$ per dozen.

Medium Bulbs, 9 to 11 inches circumference. $30 \mathrm{c}$ each; $\$ 3.00$ per dozen.

CINNAIION VINE (STRONG TUBERS). $15 \mathrm{c}$ each; $\$ 1.50$ per dozen.

MADEIRA VINE, Large Bulbs. 15c each; $\$ 1.50$ per dozen.

TUBEROSES (EXCELSIOR PEARL), Nammoth Bulbs. $12 \mathrm{c}$ each; $\$ 1.00$ per dozen.

HYACINTHUS (CANDICANS), Summer Hyacinth, Mammoth Bulbs, growing large, tall spikes bearing 20 to 30 bell-shaped, large, white flowers. $15 \mathrm{c}$ each; $\$ 1.50$ per dozen.

\section{HARDY JAPANESE LILIES.}

AURATUII, Golden Banded (Hardy). RUBRUM, ROSEUII OR MLELPIIENE (Rose or Red Striped) (Hardy). ALBUIM, SPECIOSUM (Silver Striped) (Hardy).

Price of the above on application. 


\section{FERTILIZERS}

\section{Pulverized Sheep Mianure}

Nothing can equal this for LAWNS, PLANTS, and Garr, ns, as it is the strongest of als aatura] manures, chemical analysis showing it to contain a higher per cent. of fertilizing ingredients - ammonia,

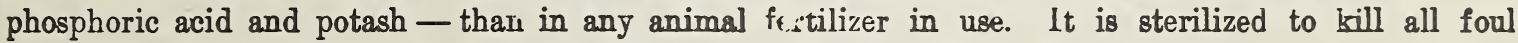
seeds and ground fine. It is clean, easily and quickly applied, has no unpleasant odor, will not kili or burn the grass like the commercial fertilizers, will not sow a crop of noxious weeds for you like Barnyard or Stable manure to fill your lawns. It is immediate in its effect and lasting.

A beautiful Lawn is a great attraction to a home and as the constant growing and cutting of grass will exhaust the soil it makes it necessary to enrich it in order to keep your lawn in a state of perfection. To do this some use Barn Manure, which is bulky, unsightly and introduces seeds of obnoxious weeds and grasses.

A few dollars epent in Sheen Manure will not only give you a beautiful lawn, but will preserve it and be a saving of money in the long run, as it is of full strength, and is very easily handled. This makes it the most economical food for lawns and plants that $t$ is possible to obtain.

FOR GARDENS, dig into the soil when snwing the seed

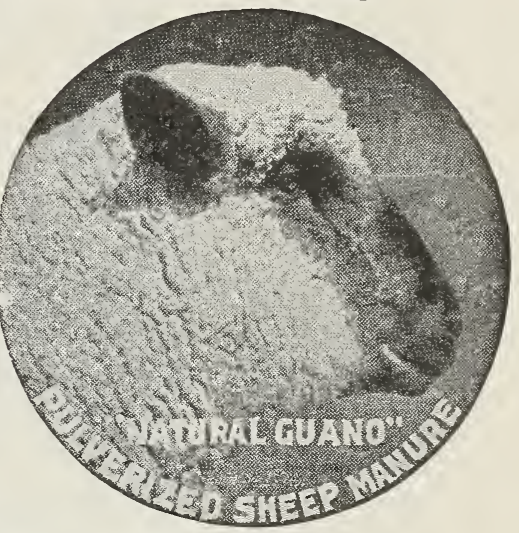
or setting out plants and it will promote a rapid strong rowth of flowers or vegetables.

FOR YOUNG TREES or BUSHES, it makes the fnest mulch, after watering it becomes a very strong solution, soaking the roots thoroughly with a needed food and they will show the good effect in a very few days after using.

FOR PLANTS and FLOWERS, it is the most nutritious food that can be applied, as a liquid dressing using five pounds to five gallons of water and may be used with perfect safety, or sprinkle it dry around the plants, working it into the ground. Remember, it will not injure the most sensitive plant but will produce healthy plants, with rich blossoms.

DIRECTIONS. Sprinkle thickly over the grass early in the Spring and once or twice in the Summer or any time during the growing season, using about $100 \mathrm{lbs}$. to 2,000 square feet, or to a space of 50 feet one way by 45 feet the other; $50 \mathrm{lbs}$. will cover 25 by 23 feet. If the season is dry, wet it down or sow just before rain. If you have only a small place it is well to buy a bag, as you can use it on your grass and gardens early and through the season as it keeps in good condition and is convenient to have on hand. Put up in $100 \mathrm{lbs}$., $50 \mathrm{lbs}$. and $25 \mathrm{lb}$. bags; $10 \mathrm{lbs}$., $5 \mathrm{lb}$. and $1 \mathrm{lb}$. packages. Prices of the above on application.

NITRATE OF SODA. Is valuable solely for the Nitrogen it contains, and is used chiefly as a s. mulant to force slow-growing grass or backward crops. It is quick in its action, forcing the plants along to maturity, and can be used alone or mixed with some sort of fertilizer.

HIGH GRADE FERTILIZER. Fertilizers are - ery high in Ammonia, Nitrogen Phosphoric Acid, and must not be classed with the low grades often called by the same name, but undesirable to grow good crops.
GROUND BONE. For Trees, Grapevines, Currant Bushes, or any thing that has heavy permanent root growth, as it is slow in effect.

ACID PHOSPHATE AND HUMIS.

BOWKER'S PLANT FOOD. For House Plants, Palms, Ferns, etc.

FERTILIZERS are put up in $100 \mathrm{lb}$, $50 \mathrm{lb}$. $25 \mathrm{lb}$. bags, and in $10 \mathrm{lb}$. and $5 \mathrm{lb}$. packages. 


\section{DISINFECTANTS AND INSECTICIDES FOR SPRAYING.}

When ordered by mail add postage at Parcel Post Rates. Liquids cannot be sent by mail.

Spraying is no longer an experiment; it is an accepted practice, if you desire to grow Perfect Vegetables, Fruit or Flowers. If you wish success with fruit you will have to spray your trees. It is impossible to raise fruit successfully without spraying. Spray, not sprinkle, until the solution begins to drip from the leaf.

WHEN TO SPRAY.- The time of making the application varies with the season. As a rule, for garden fruits, when the insects first appear; for orchard fruits, or the more common insects, just before the fruit buds open, and just after the blossoms fall. Never spray a tree when in blossom.

With the special pumps and prepared insecticides now on the market, the labor of successfully combating with nearly all injurious insects is very easy. We carry in stock the very best and most complete line of insecticides, also, fungicides, spray pumps, hose and rozzles.

\section{INSECTICIDES}

HAMMOND'S SLUG SHOT.

HAMMOND'S GRAYE DUST.

HAMMOND'S KEROSENE EMULSION. DRY BORDEAUX AND PARIS GREEN COMPOUND.

POWDERED SULPHUR.

BOWKER'S PYROX.

ARSENATE OF LEAD - Paste and Dry. PURE PARIS GREEN.

HELLEBORE.

SCALECIDE.

LIME AND SULPHUR - Liquid and Dry.

DRY BORDEAUX MIXTURE-Prevents blight on all crops.

FRENCH BORDEAUX PASTE-For rust and rot.

BLACK LEAF 40. Sulphate of Nicotine.-It is highly recommended for Pear Thrip, Pear Psylla, Hop Louse, Aphis, etc. Also for Sheep and Cattle Dipping. Directions on package.

NICO FUME PAPER-This is the strongest Tobacco Paper on the market. Does not injure blooms and furnishes the easiest method for fumigation ever devised. Especially destructive to Aphides or Green Fly, Thrips, and all insects. Cans, containing 24 sheets, 144 sheets, 288 sheets.

NICO FUME LIQUID.-A very effective vaporizing liquid for spraying plants and fumigating greenhouses.

FIR-TREE-OIL SOAP - For destroying Lice, Red Spider, Mealy Bugs, Scale, Aphis.

FISH OR WHALE-OIL SOAP - For the destruction of insects on plants.

PLANT LICE KILLER.

ARSENATE OF LEAD AND BORDEAUX - Dry and Paste.

BUG DEATH can be applied wet or dry.

TOBACCO DUST.

ZINC BORDEAUX.

\section{INSECTICIDES-Cont.}

CUT WORM KILLER.

ANTICIDE-For destroying Ants on lawns and in vegetable crops.

MAGOTITE-A real root maggot remedy. It is not injurious to plant life. It is a sure remedy for the root maggot.

PERFECTION FLY KII LER.

\section{DISINFECTANTS}

B. \& B. DISINFECTANT - Lice Killer and Fly Exterminator.

CARBOLA - Use it instead of White Wash and Disinfectants.

CARBOLINEUM - A spiendid disinfectant and wood preservative.

HAMMOND'S SHEEP WASH.

PRATT'S DIP AND DISINFECTANT.

CONKEY'S NOXICIDE.

\section{SUNDRIES}

GRAFTING WAX.

STERLINGWORTH WEED KILLER - Will remove the weeds from Tennis Courts, Driveways. and Garden Paths.

PERMANITE - The Modern Material for Greenhouse and Sash Glazing. Safe, Quick and Lasting.

HOT BED MATS.

THERMOMETERS, hot bed, green house. dairy and incubator.

FARMOGERM.

PRUNING TOOLS.

GRAFTING TOOLS.

LAWN MOWERS. 


\section{Poultry Supplies and Remedies}

Agents for Buckeye Incubators and Hovers. Standard Coal Burning Hovers. Cyphers Incubators, Brooders and Portable Hovers. Reliable Incubators and Oil Heated Blue Flame Brooders.

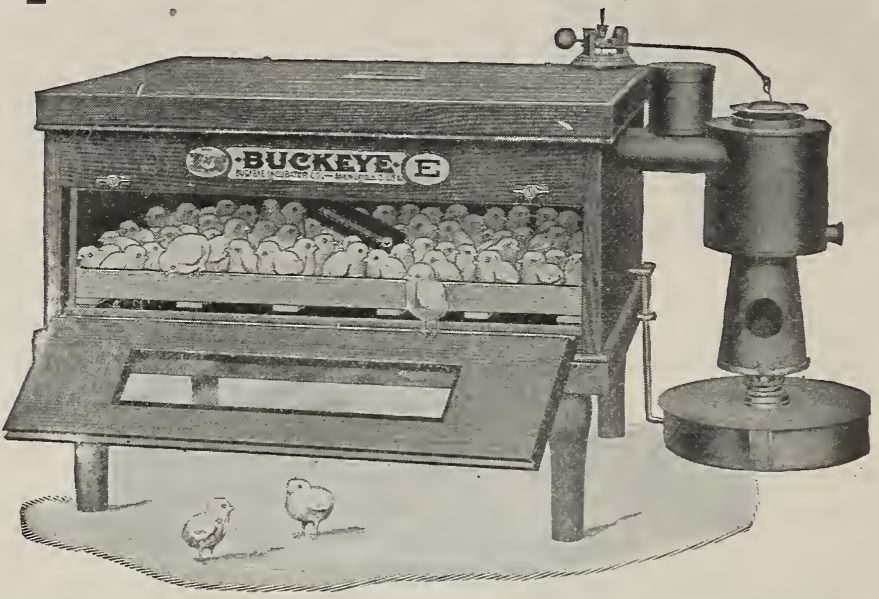

Drinking Fountains, Sexton Hoppers, Dry Food Hoppers, Chick Servers, Chick Fountains, Chick Feeders, Grit and Shell Hoppers. Roup Remedies: Rust's, Pratt's and Conkey's. Lice Powder: Rust's, Pratt's, Conkey's, Smith's and Lambert's Death to Lice, Liquid Lice Killer and Disinfectants, Scratch Feed, Chick Feed, Chick Starting Feed, Grit, Shell, Charcoal, Cut Clover, Hen-E-Ta, Ground Bone, Beef Scrap, Incubator and Brooder Thermometers, Leg Bands, Poultry Punches, Parcel Post Egg Boxes, Egg Cartons, Egg Carriers, and Egg Testers.

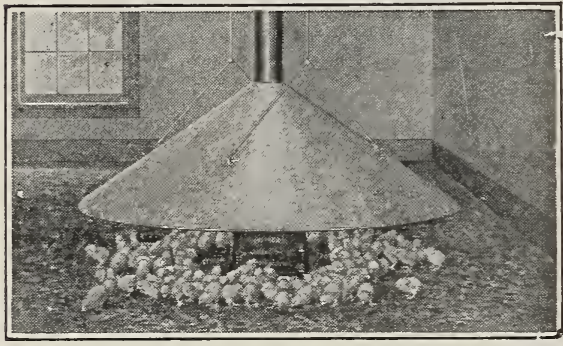

Standard Coal-Burning Hover.

\section{We Carry a Full Line for the Poultry Keeper}

Standard Coal-Burning Colony Hover. - To meet the demand for a low-priced colony hover, we offer the Standard Hover. It is practical, reliable and serviceable, and is the best colony hover selling at the price. Two sizes, 300 chicks to 1,000 chicks.

The Buckeye Portable Hover is not a center heated Hover but is warmest near the curtain. The chicks will distribute themselves around the edge of the curtain, where they have plenty of room and fresh air as well as warmth, with no crowding to the center. The flame cannot be put out by wind or rain, there being no connection between the lamp chimney

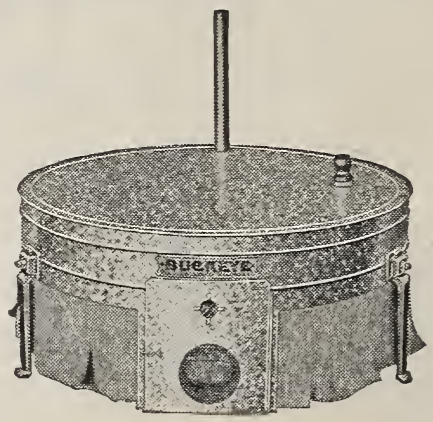
and the flues for taking in fresh air and discharging gases.

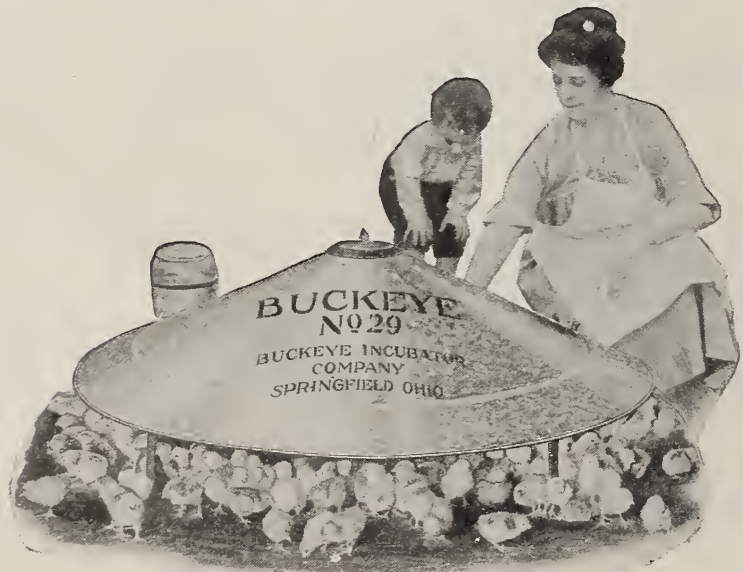

\section{BUCKEYE " BLUE FLAME" BROODERS}

By using these you save time, money and trouble and your chickens will grow faster, get heavier and thrive, because our Brooder will take the very best care of them and keep them comfortable.

These Brooders are built in three sizes: 34-inch canopy for 200 chicks; 42-inch canopy for 350 chicks; 52-inch canopy for 500 chicks. Operates on same successful principle as the standard Coal Burning Brooder. 


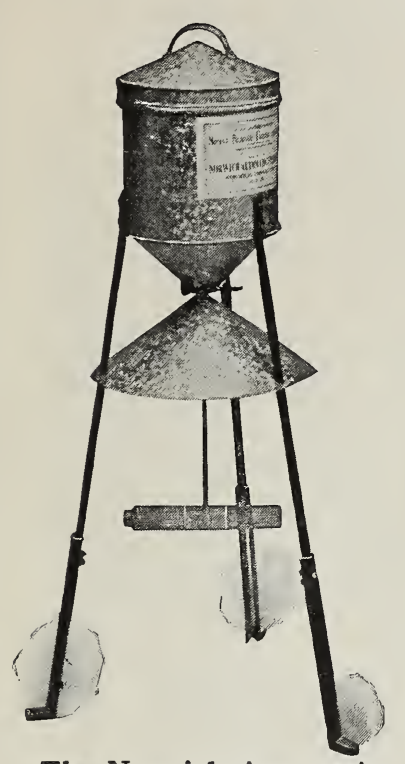

The Norwich Automatic Exer iser and Feeder

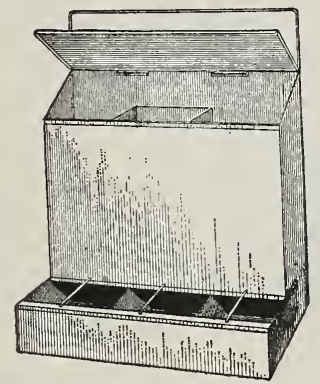

Grit and Shell Hopper

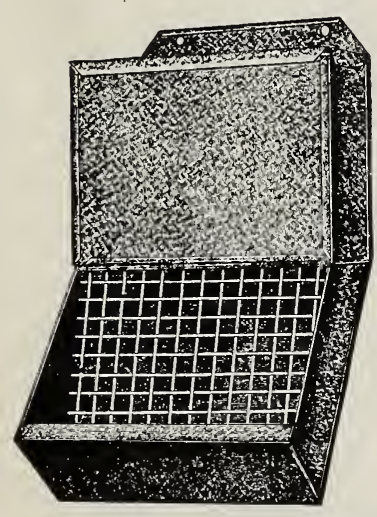

Sexton Mash Hopper

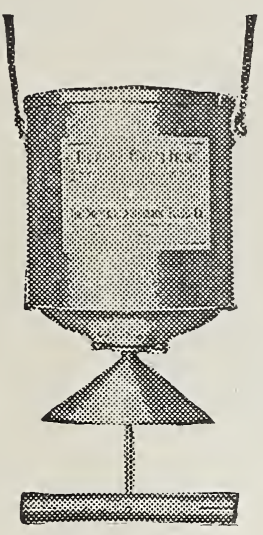

The Apaco Poultry Feeder

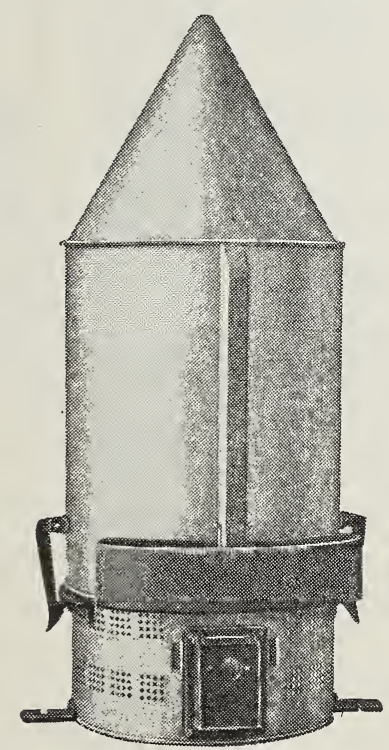

The Norwich Automatic Air-Lock Fountain

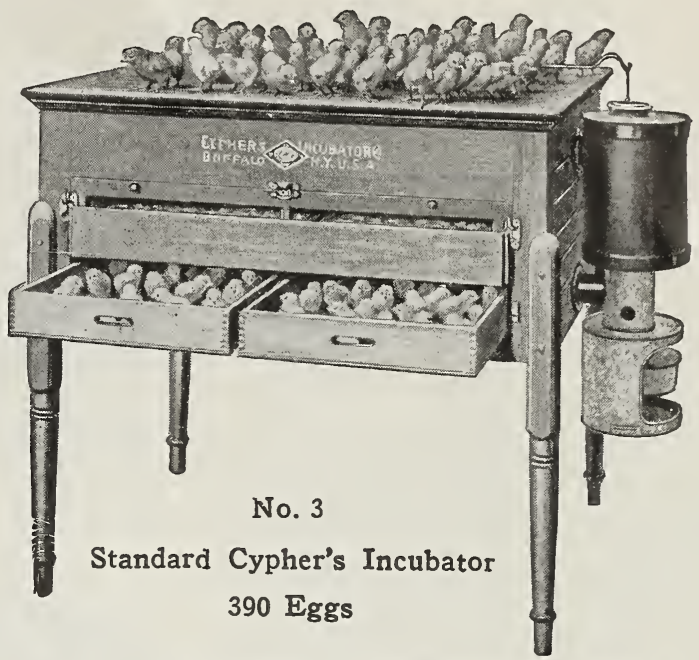

\section{Revolution in Chick Raising}

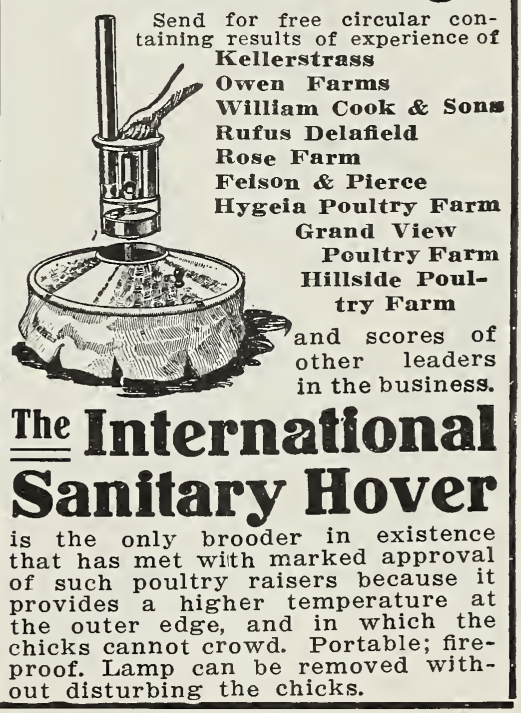

PRATT'S BUTTERMILK BABY CHICK FOOD PRATT'S BUTTERMILK GROWING MASH PRATT'S WHITE DIARRHEA REMEDY PRATT'S POULTRY REGULATOR PRATT'S DIP AND DISINFECTANT PRATT'S ROUP CURE PRATT'S POWDERED LICE KILLER PRATT'S RED MITE SPECIAL CARTONS FOR CARRYING EGGS PARCEL POST BOXES FOR SHIPPING EGGS

MALTOID MILK BONE, DOG AND PUPPY BISCUIT. It is a scientific combination of the food properties of malt and milk, wi: . other ingredients used. These produce a food rich in proteid, and, having the proper amount of carbohydrates to constitute a complete food, it aids digestion, prevents foul breath and decayed teeth, imparts vitality, and improves the glossiness of the coat. It is a very powerful nourisher and muscle builder, aids in the assimilation of other food, and is, itself, capable of almost complete assimilation. 


\section{Poultry Supplies Everything for the Poultry Keeper}

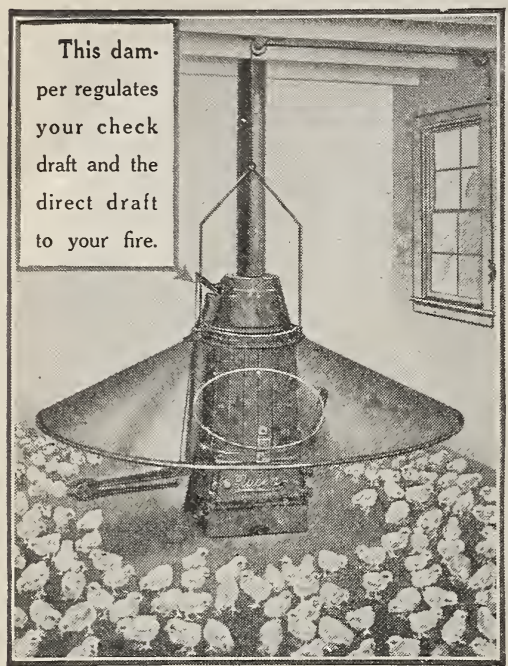

QUEEN BROODER STOVE

No. 1. Capacity 500 Chicks. \$21.50 No. 2. Capacity 1000 Chicks. 26.50

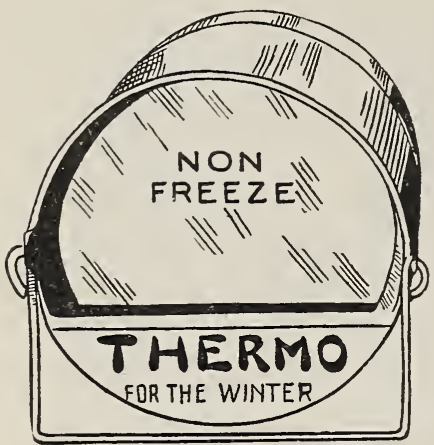

THERMO FOUNTS

Double Wall - Non-Freezing

2 Gallon ............... \$2.50

3 Gallon $\ldots \ldots \ldots \ldots \ldots \ldots \ldots \ldots \ldots \ldots, 3.00$

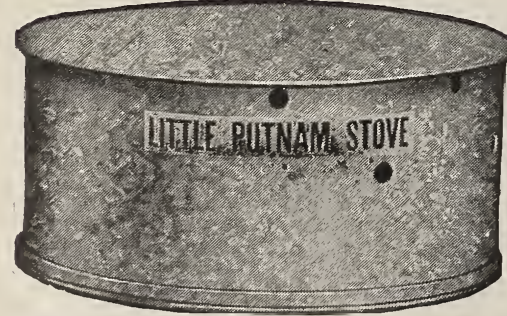

THE LITTLE PUTNAM STOVE

Used under the drinking vessels of the Poultry House to keep the water from freezing in the coldest weather. 2.00

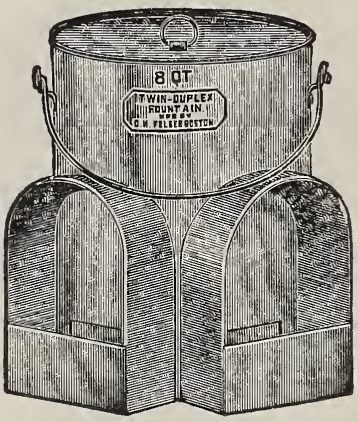

Eight qt. Twin Duplex

8 qt. size, each $\$ 1.65$

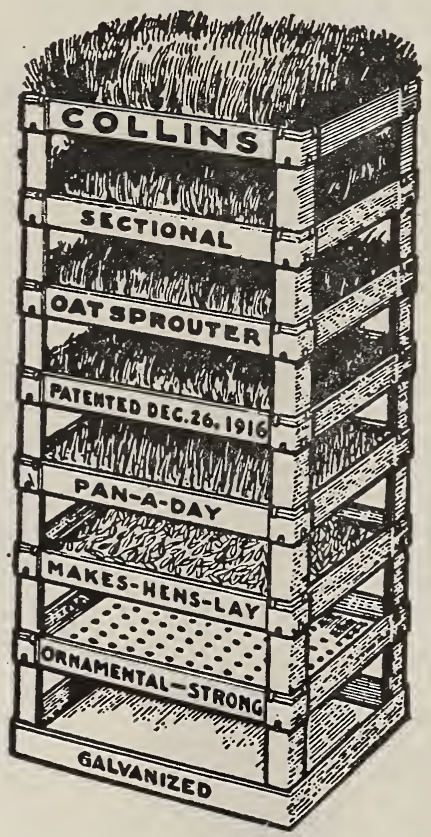

FIRELESS OAT SPROUTER

5 Pan ............ \$3.00
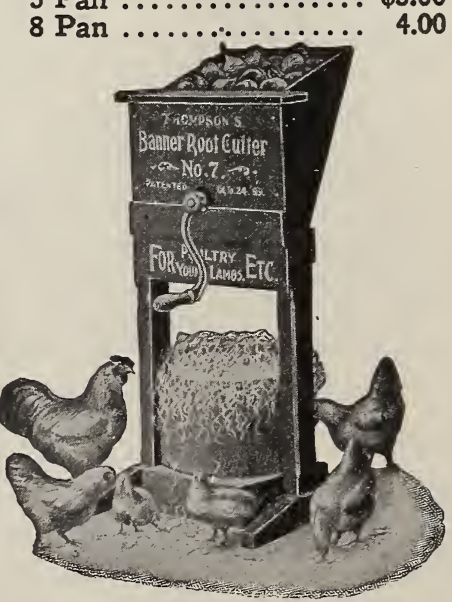

ROOT CUTTER FOR POULTRYMEN

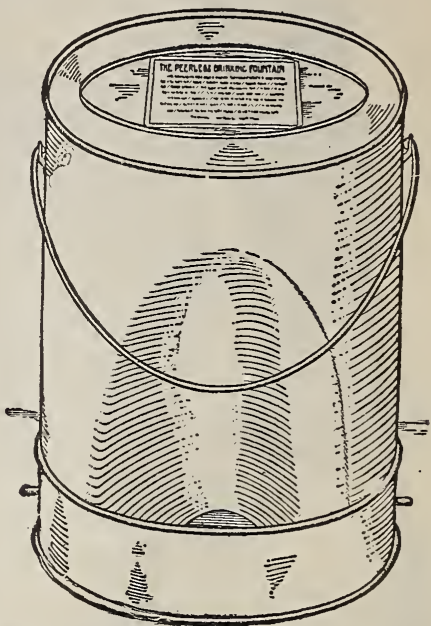

PEERLESS SANITARY FOUNTS 5 Quart ................\$.90 10 Quart ................. 1.25
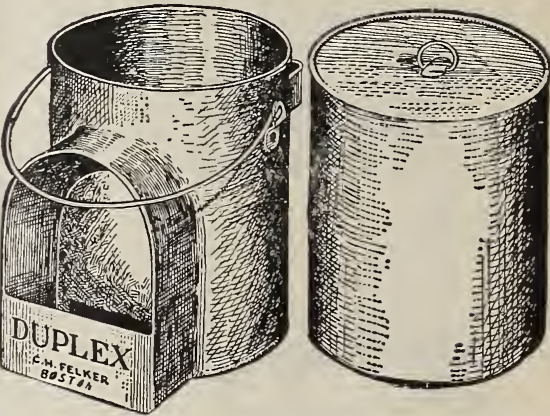

\section{DUPLEX SANITARY FOUNTS}

4 Quart ................\$ \$1.10

8 Quart ................ 1.40

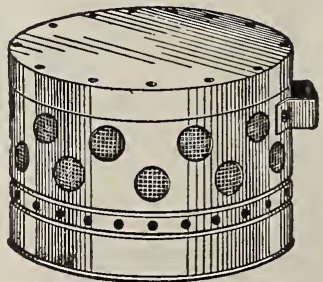

THE PEERLESS HEATER

Can be used with any fountain. $\$ 1.75$

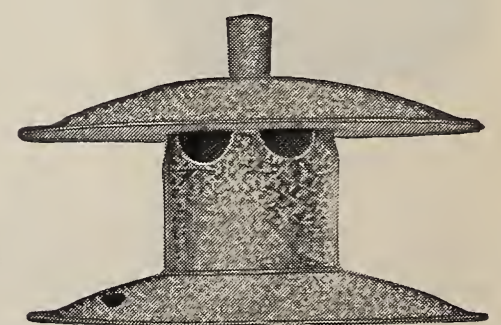

PUTNAM BROODER HEATER Price, \$4.75, postpaid.

BABY CHICKS FOR SALE IN MARCH, APRIL AND MAY 
RATE TABLE FOR PARCEL POST SHIPMENTS

\begin{tabular}{|c|c|c|c|c|c|c|c|c|c|}
\hline \multicolumn{3}{|c|}{$\begin{array}{l}\text { First Zone } \\
\text { up to } \\
50 \text { miles } \\
\text { LOCAL RATE } \\
\text { ZONE }\end{array}$} & \multirow{2}{*}{ 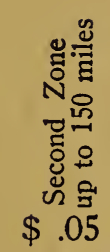 } & \multirow{2}{*}{ 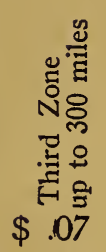 } & \multirow{2}{*}{ 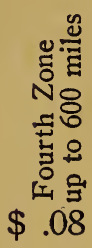 } & \multirow{2}{*}{ 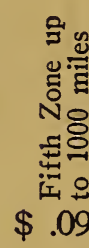 } & \multirow{2}{*}{ 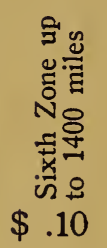 } & \multirow{2}{*}{ 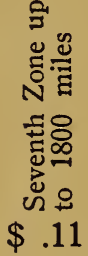 } & \multirow{2}{*}{ 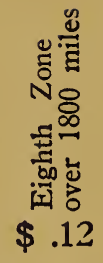 } \\
\hline 1 & $\$ .05$ & $\$ .05$ & & & & & & & \\
\hline 2 & .06 & .06 & .06 & .12 & .14 & .1 & .19 & .21 & .24 \\
\hline 3 & .06 & .07 & .07 & .17 & .20 & .2 & .28 & .31 & .36 \\
\hline 4 & .07 & .08 & .08 & .22 & .26 & .3 & .37 & .41 & .48 \\
\hline 5 & .07 & .09 & .09 & .27 & .32 & .3 & .46 & .51 & .60 \\
\hline 6 & .08 & .10 & .10 & .32 & .38 & .4 & .55 & .61 & .72 \\
\hline 7 & .08 & .11 & .11 & .37 & .44 & .5 & .64 & .71 & .84 \\
\hline 8 & .09 & .12 & .12 & .42 & .50 & .5 & .73 & .81 & .96 \\
\hline 9 & .09 & .13 & .13 & .47 & .56 & .6 & .82 & .91 & 1.08 \\
\hline 10 & .10 & .14 & .14 & .52 & .62 & .7 & .91 & 1.01 & 1.20 \\
\hline 11 & .10 & .15 & .15 & .57 & .68 & .7 & 1.00 & 1.11 & 1.32 \\
\hline 12 & .11 & .16 & .16 & & & & & & \\
\hline 13 & .11 & .17 & .17 & & & & & & \\
\hline 14 & .12 & .18 & .18 & & & & & & \\
\hline 15 & .12 & .19 & .19 & & & & & & \\
\hline 16 & .13 & .20 & .20 & & & & & & \\
\hline 17 & .13 & .21 & .21 & & & & & & \\
\hline 18 & .14 & .22 & .22 & & & & & & \\
\hline 19 & .14 & .23 & .23 & & & & & & \\
\hline 20 & .15 & .24 & .24 & & & & & & \\
\hline
\end{tabular}




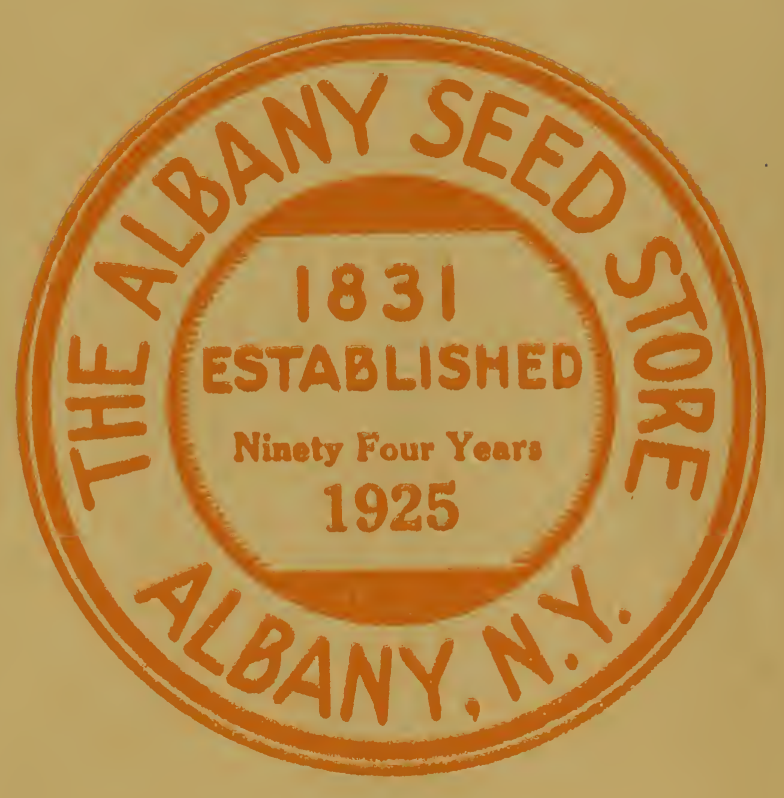

Our New Location

66 Beaver St. Cor. of William St.

One Short Block from South Pearl Street Half A Block From Public Market 